U.S. DEPARTMENT OF COMMERCE

National Bureau of Standards

NBS

PUBLICATIONS

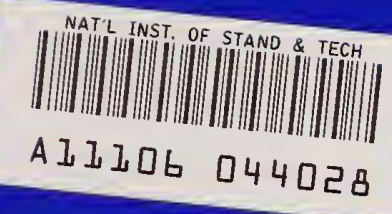

NBS Technical Note 1212

A Computerized Fracture

Mechanics Database for

Oxide Glassses

S.W. Freiman, T.L. Baker, and J.B. Wachtman, Jt:

Library

National Bureau

of Standards

JUL 311985

NBSIDSS NBS NBS NBS NBS NBS NBS $B S N B S N B S N B S N B S$ NBS NBS NBS NB:

IS NBS NBS NBS NBS NBS NBS NBS NBS NBS NB.

NBS NBS NBS NBS NBS NBS NBS NBS NBS NBS

is NBS NBS NBS NBS NBS NBS NBS NBS NBS NB:

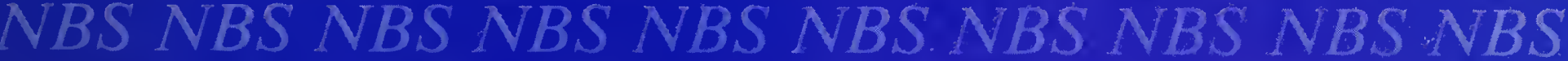

iS NBS NBS NBS NBS NBS NBS NBS NBS NBS NB

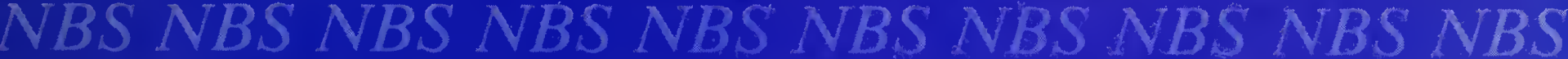

iS NBS NBS NBS NBS NBS NBS NBS NBS NBS NB

NBS NBS NBS National Bureau of Standards NBS NBS

iS NBS NBS NBS NBS NBS NBS NBS NBS NBS NB

$Q C$

100

BS NBS NBS NBS NBS NBS NBS NBS NBS NB:

.05753

1212

$3 S$ NBS NBS NBS NBS NBS NBS NBS NBS NB

1985 
he National Bureau of Standards ${ }^{1}$ was established by an act of Congress on March 3, 1901. The Bureau's overall goal is to strengthen and advance the nation's science and technology and facilitate their effective application for public benefit. To this end, the Bureau conducts research and provides: (1) a basis for the nation's physical measurement system, (2) scientific and technological services for industry and government, (3) a technical basis for equity in trade, and (4) technical services to promote public safety. The Bureau's technical work is performed by the National Measurement Laboratory, the National Engineering Laboratory, the Institute for Computer Sciences and Technology, and the Center for Materials Science.

\section{The National Measurement Laboratory}

Provides the national system of physical and chemical measurement; coordinates the system with measurement systems of other nations and furnishes essential services leading to accurate and uniform physical and chemical measurement throughout the Nation's scientific community, industry, and commerce; provides advisory and research services to other Government agencies; conducts physical and chemical research; develops, produces, and distributes Standard Reference Materials; and provides calibration services. The Laboratory consists of the following centers:
- Basic Standards ${ }^{2}$

- Radiation Research

- Chemical Physics

- Analytical Chemistry

\section{The National Engineering Laboratory}

Provides technology and technical services to the public and private sectors to address national needs and to solve national problems; conducts research in engineering and applied science in support of these efforts; builds and maintains competence in the necessary disciplines required to carry out this research and technioal service; develops engineering data and measurement capabilities; provides engineering measurement traceability services; develops test methods and proposes engineering standards and code changes; develops and proposes new engineering practices; and develops and improves mechanisms to transfer results of its research to the ultimate user. The Laboratory consists of the following centers:
- Applied Mathematics

- Electronics and Electrical Engineering ${ }^{2}$

- Manufacturing Engineering

- Building Technology

- Fire Research

- Chemical Engineering ${ }^{2}$

\section{The Institute for Computer Sciences and Technology}

Conducts research and provides scientific and technical services to aid Federal agencies in the selection, acquisition, application, and use of computer technology to improve effectiveness and economy in Government operations in accordance with Public Law 89-306 (40 U.S.C. 759), relevant Executive Orders, and other directives; carries out this mission by managing the Federal Information Processing Standards Program, developing Federal ADP standards guidelines, and managing Federal participation in ADP voluntary standardization activities; provides scientific and technological advisory services and assistance to Federal agencies; and provides the technical foundation for computer-related policies of the Federal Government. The Institute consists of the following centers:
- Programming Science and Technology

- Computer Systems Engineering

\section{The Center for Materials Science}

Conducts research and provides measurements, data, standards, reference materials, quantitative understanding and other technical information fundamental to the processing, structure, properties and performance of materials; addresses the scientific basis for new advanced materials technologies; plans research around cross-country scientific themes such as nondestructive evaluation and phase diagram development; oversees Bureau-wide technical programs in nuclear reactor radiation research and nondestructive evaluation; and broadly disseminates generic technical information resulting from its programs. The Center consists of the following Divisions:
- Inorganic Materials

- Fracture and Deformation ${ }^{3}$

- Polymers

- Metallurgy

- Reactor Radiation 
A Computerized Fracture

Mechanics Database for Oxide Glasses

S.W. Freiman and T.L. Baker

Inorganic Materials Division

Center for Materials Science

National Bureau of Standards

Gaithersburg, Maryland 20899

J.B. Wachtman, Jr.

Rutgers University

Center for Ceramics Research

Piscataway, New Jersey 08854

Issued June 1985

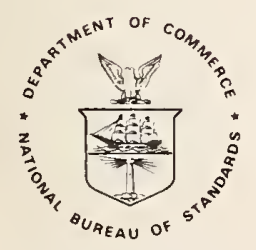

U.S. Department of Commerce Malcolm Baldrige, Secretary

National Bureau of Standards Emest Ambler, Director 
National Bureau of Standards

Technical Note 1212

Natl. Bur. Stand. (U.S.)

Tech. Note 1212,

9l pages (June 1985)

CODEN: NBTNAE
U.S. Government Printing Office

Washington: 1985
For sale by the Superintendent of Documents,

U.S. Government Printing Office. Washington, DC 20402 


\section{Contents}

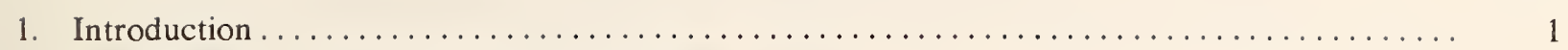

2. Design of the Fracture Mechanics Data Base $\ldots \ldots \ldots \ldots \ldots \ldots \ldots \ldots$

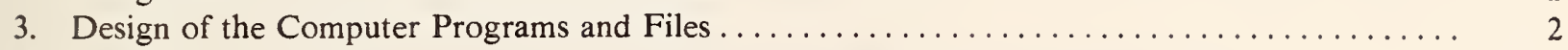

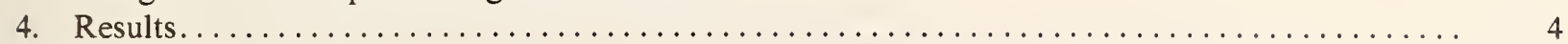

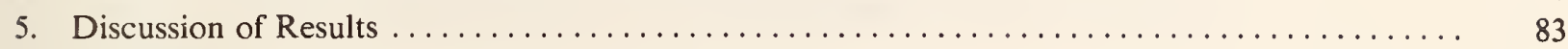

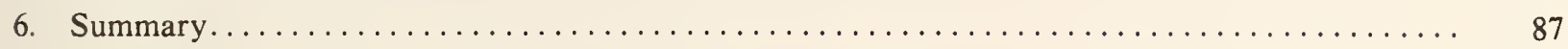





\title{
A Computerized Fracture Mechanics Database for Oxide Glasses
}

\author{
S. W. Freiman and T. L. Baker \\ Inorganic Materials Division, National Bureau of Standards, Gaithersburg, MD 20899 \\ and \\ J. B. Wachtman, Jr. \\ Rutgers University, Center for Ceramics Research. Piscataway, NJ 08854
}

\begin{abstract}
Values of critical fracture toughness $\left(K_{\mathrm{IC}}\right)$, fracture energy $(\gamma)$, subcritical crack growth exponents $(n)$ and Young's modulus $(E)$, are compiled and tabulated for a wide variety of oxide glasses. A computerized data retrieval system has been formulated to allow for selection of data by either glass composition, investigator, or experimental technique, and year. Plotting routines allow $K_{\mathrm{IC}}$ or $\gamma$ to be plotted versus either the mole \% of a particular component or the Young's modulus of the glass. A few illustrations are given to demonstrate trends in $K_{\mathrm{IC}}$ and $\gamma$ as a function of composition and elastic modulus.
\end{abstract}

Key words: crack growth exponents; elastic modulus; fracture database; fracture mechanics; fracture toughness; oxide glasses.

\section{Introduction}

The strength of glass and other brittle materials has long been known to depend on the size and character of flaws. The strength of a particular specimen of glass depends on both the severity of the most serious flaw (an extrinsic property of the glass) and the resistance of the glass to flaw propagation (thought to be an intrinsic property of the glass). The theory of linear elastic fracture mechanics predicts that for a simple crack of length $C$ the strength $S$ will be given by

$$
S=K_{\mathrm{I}} /\left(Y C^{1 / 2}\right)
$$

where $Y$ is a numerical constant that depends on flaw location and geometry and $K_{\text {IC }}$ is the critical stress intensity factor, usually taken to be an intrinsic property of the material.

An entire theory and methodology of design has been developed for brittle materials based on linear elastic fracture mechanics. It is recognized that $K_{\mathrm{IC}}$, will change with glass composition. However, all of the aspects of composition which affect the critical stress intensity factor are not sufficiently well understood to achieve the goal of being able to predict those glasses which have optimum fracture toughness as well as possess the other properties required by the application. Accordingly, there is both scientific and technological interest in examining the whole body of fracture mechanics data for glasses to see how well critical stress intensity values can be understood in terms of the character of the glasses.

The foregoing discussion relates to cases in which no environmentally enhanced slow crack propagation occurs prior to sudden failure, i.e. in inert environments, or to rapid-fracture measurements in which any slow crack propagation has a minimal effect on the strength. The phenomenon of moisture-assisted slow crack growth occurs to some extent in every oxide glass of which we are aware, and so must be accounted for in structural design. The rate of crack propagation is a strong function of the stress intensity factor, $K_{\mathrm{l}}$, at the flaw. The external factors of crack size and shape can be taken into account through eq (1). An equation widely used to fit crack growth data over large ranges of crack propagation rates is:

$$
V=A K_{\mathrm{I}}{ }^{n}
$$

where $V$ is the crack velocity and $A$ and $n$ are empirical constants that depend on glass composition as well as the external environment. A methodology for safe-life design with materials that undergo slow crack propaga- 
tion has been developed based on eqs (1) and (2). Again it is recognized that the constants $A$ and $n$ may vary if the composition varies within a family of glasses. As before, it is hoped that the factors affecting the above constants can be sufficiently understood, specified, and controlled to permit reliable engineering use.

The time is ripe for the development of a comprehensive compilation of the available fracture mechanics data on glasses. On one level, it is appropriate to inquire if the accumulated data are sufficiently consistent to form a useful guide in the choice of glass compositions for load-bearing applications. On another level, one can ask if the existing data show trends, such as dependence on composition, useful in developing new glasses or in further understanding fracture behavior. In addition, such a compilation is the first step in evaluating the quality of this data. The present paper describes a computerized fracture mechanics data base and associated computer programs which permit extension and modification of the data base as well as selection, plotting and curve fitting. Some preliminary results of correlations of fracture energy, $\gamma$, and critical stress intensity factor $K_{1 \mathrm{c}}$ with composition and elastic modulus are presented.

\section{Design of the Fracture Mechanics Data Base}

The form of the fracture mechanics data base was designed with several ideas in mind. The following information was included:

1. Primary parameters such as $K_{1 \mathrm{C}}, n$, etc., required for engineering design for glass structure.

2. Secondary parameters, e.g. elastic modulus.

3. Test methods and environmental factors.

4. Manufacturers and engineering designations for commercial glasses.

5. References.

The design is intended to be as open-ended as possible and the data base should be capable of expansion in the number of records it contains. ${ }^{1}$ The data base should be computer searchable in detail; i.e., for each record, each type of information is formatted to be available for analysis without further personal judgment being required. The data base is in a form generally accessible for computer research by other users; i.e., it is in a format and language generally available and likely to remain in use.

For this data base, fracture energy and the related quantity, critical fracture toughness, were chosen as the primary variables. The slow crack-propagation constant, $n$, and Young's modulus, $E$, were also compiled when available. A survey of published literature as well as private sources, i.e., government reports and corporate data, suggested that about 300 measurements are available on reasonably well-characterized glasses. For each measurement, the number of associated items making up a record can be as large as 25 . An expansion of a factor of 2 in the number of records in the next few years seems possible, and was taken as a data base design goal. The resulting size, though substantial in terms of data bases to be manually developed and analyzed, is not very large by computer standards. Flexibility rather than efficiency in use of computer memory or speed was therefore taken as the primary goal.

\section{Design of the Computer Programs and Files}

In anticipation of possible future use by a variety of persons with minimal programming knowledge, all programs were written in menu-driven style and in a simple language (Basic). In anticipation of possible use in small organizations which might lack large center computers, the programs and files were subdivided into units which can be run on the current generation of stand-alone laboratory microcomputers. That is, the computer only needs Basic, a minimum of 128 kilobytes of memory, and floppy disk storage of at least 160 kilobytes per diskette. To keep within these limits, the file-creating program was kept separate from the file-analysis program.

The file creating program has been designed to ask the keyboard operation for the following information for each record:

1. The record number

2. The first previous record number for the same glass

3. A generic description of the glass (e.g., aluminosilicate)

4. A specific description of the glass (e.g., a commercial specification) 'The term record is taken to mean the complete set of information associated with each independent measurement of a fracture mechanics
parameter. 
5. Manufacturers name when appropriate

6. Indication of whether composition will be input in mole percent $(M)$ or weight percent $(W){ }^{2}$

7. The percentage of each of the following in mole or weight percent:
a. $\mathrm{Al}_{2} \mathrm{O}_{3}$
b. $\mathrm{B}_{2} \mathrm{O}_{3}$
c. $\mathrm{CaO}$
d. $\mathrm{MgO}$
e. $\mathrm{Na}_{2} \mathrm{O}$
f. $\mathrm{K}_{2} \mathrm{O}$
g. $\mathrm{PbO}_{2}$
h. $\mathrm{SiO}_{2}$

8. The percentage of any two other oxides specified by the user

9. Young's modulus in units of $\mathrm{MPa}$

10. The fracture mechanics data itself:

a. the test method (see table 1 for abbreviations used for the test methods).

b. the critical stress intensity in units of $\mathrm{MPa} \mathrm{m} \mathrm{m}^{1 / 2}$. $^{3}$

c. the fracture energy in units of $\mathrm{J} / \mathrm{m}^{2}{ }^{3}$

11. Same as 10 but for a second measurement method when appropriate.

12. The crack growth exponent, $n$.

13. The test environment. ${ }^{4}$

14. The reference.

15. Comments.

Table 1. Abbreviations used in the computerized data base for the test methods used.

\begin{tabular}{ll}
\hline \hline Abbreviation & \multicolumn{1}{c}{ Test method } \\
\hline NB & Notched Beam \\
DCB & Double Cantilever Beam \\
AMDCB & Applied Moment Double Cantilever Beam \\
DT & Double Torsion \\
Ind. Crk. Len. & Indentation Crack Length \\
Controlled Flaw & Controlled Flaw \\
Static Fat. & Static Fatigue \\
Dynamic Fat. & Dynamic Fatigue \\
Short bar & Short Bar \\
\hline
\end{tabular}

Table 2. List of environments used by various authors

air
air, $x \% \mathrm{RH}$ (i.e. air with $x \%$ relative humidity)
$\mathrm{H}_{2} \mathrm{O}$
$\mathrm{D} . \mathrm{I} . \mathrm{H}_{2} \mathrm{O}$ (i.e. deionized water)
$\mathrm{N}_{2}$ (l) at $-196^{\circ} \mathrm{C}$ (i.e. liquid nitrogen at $-196^{\circ} \mathrm{C}$ )
$\mathrm{Heptane}$
$\mathrm{N}_{2}$ (l) (i.e. liquid nitrogen)
$\mathrm{N}_{2}$ (g) (i.e. nitrogen gas)
$\mathrm{Vacuum}$
Toluene
$\mathrm{Mineral}$ oil
$1 \mathrm{M} \mathrm{Cs}$ in D.I. $\mathrm{H}_{2} \mathrm{O}$ (i.e. $1 \mathrm{Molar} \mathrm{Cs}$ in deionized water)
$1 \mathrm{M} \mathrm{Li}$ in D.I. $\mathrm{H}_{2} \mathrm{O}$ (i.e. $1 \mathrm{Molar} \mathrm{Li}$ in deionized water)
$6 \mathrm{~N} \mathrm{NaOH} \mathrm{(i.e.} 6 \mathrm{Normal} \mathrm{NaOH}$ )
$6 \mathrm{~N} \mathrm{HCl}$ (i.e. $6 \mathrm{Normal} \mathrm{HCl}$ )

${ }^{2}$ When the input is in weight percent, a subroutine in the program enables the user to convert these values to mole percent, and a $\mathrm{C}$ is placed in Item 6 to indicate the calculation has been done.

${ }^{3}$ When either b or $\mathrm{c}$ alone is given and a value for Young's modulus is present, the program calculates the other from the identity $\left(K_{\mathrm{IC}}=\right.$ $2 E \gamma)^{1 / 2}$ where $E$ is Young's modulus.

${ }^{4}$ Both the relative humidity of air, the chemical activity of water in solutions and the $\mathrm{pH}$ value in solutions are known to affect the slow crack propagation parameters and may affect other fracture mechanics parameters unless the test is conducted very rapidly. The various environments that have been used are listed in table 2. 
To reduce effort in keyboarding, the program allows duplication of earlier data (such as the reference) by a single keystroke when appropriate. Each record is written to permanent storage on a diskette before entry of the next record.

The file-analysis program as currently written provides for a search of the file for any combination of the following keys:

1. Generic material

2. Specific material designation

3. Presence of a first specified oxide

4. Presence of a second specified oxide

5. Presence of a third specified oxide

6. Test techniques

7. Principal author

8. Year

Following the search and selection of the appropriate data, this program provides the option of plotting with the critical stress intensity factor, the fracture energy, or the crack velocity parameter as the independent variable. Any of these parameters may be plotted as a function of Young's modulus, or the mole percentage of one of the specified oxides. Provision for fitting and plotting a straight line or a quadratic least squares fit to the data is also provided. Also, Young's modulus may be plotted as a function of composition.

\section{Results}

The current data base consists of 291 separate records, some of which contain measurements of $\gamma$ or $K_{\mathrm{IC}}$ obtained by more than one technique. In some instances, a record will contain a value of the crack growth exponent, $n$, instead of $\gamma$ or $K_{\mathrm{IC}}$. The entire list of records is given in table 3. Because table 3 is not ordered with respect to composition, author etc., finding a specific set of data could be difficult. Therefore, we have constructed additional tables 4-7 which contain the four major compositional categories of these glasses, i.e. silicate $\left(\mathrm{SiO}_{2}\right)$, borate $\left(\mathrm{B}_{2} \mathrm{O}_{3}\right)$, phosphate $\left(\mathrm{P}_{2} \mathrm{O}_{3}\right)$, and germanate $\left(\mathrm{GeO}_{2}\right)$. Within each table the data is listed in order of decreasing amounts of the primary constituent of the glass. At the end of each different glass composition is the reference indicating the source of the data. Each reference, which is included in its entirety in table 3, has been abbreviated and is broken down in the following manner: "WIE74/1" refers to a paper which has Wiederhorn as the primary author and is the first of two papers written by him in 1974. If there is only one paper by a particular author in one year, no /\# will be suffixed to the reference. 
Table 3. Fracture mechanics parameters for oxide glasses

ASH82

Specific Material =

Manufacturer $=$

$\% \mathrm{Al}_{2} \mathrm{O}_{3}=$

$\% \mathrm{~B}_{2} \mathrm{O}_{3}=$

$\% \mathrm{~K}_{2} \mathrm{O}=$

$\% \mathrm{Na}_{2} \mathrm{O}=$

Other Formula $=\mathrm{P}_{2} \mathrm{O}_{5}$

Other $\%=50$

Other $\%=$

Other Formula =

Young's Modulus $=5.85 E 4$

1st Technique $=$ Ind. Crk. Len.

$K_{\text {IC }}=.7$

$\% \mathrm{CaO}=50$

$\% \mathrm{MgO}=$

$\% \mathrm{PbO}=$

$\% \mathrm{SiO}_{2}=$

2nd Technique $=$

$K_{\mathrm{IC}}=$

Gamma $=4.19$

$n=$

Environment $=$ air

Reference = ASHIZUKA,M.,BRADT,R., JACTAW, Vol. 65, No. 5, 1982

Comments $=$

Specific Material =

Manufacturer =

$\% \mathrm{Al}_{2} \mathrm{O}_{3}=$

$\% \mathrm{~B}_{2} \mathrm{O}_{3}=$

$\% \mathrm{CaO}=$

$\% \mathrm{PbO}=$

$\% \mathrm{Na}_{2} \mathrm{O}=$

$\% \mathrm{~K}_{2} \mathrm{O}=$

Other Formula $=\mathrm{SrO}$

Other $\%=50$

Other Formula $=\mathrm{P}_{2} \mathrm{O}_{5}$

Other $\%=50$

Gamma =

Young's Modulus $=5.0 \mathrm{E} 4$

1st Technique $=$ Ind. Crk. Len.

$K_{\mathrm{IC}}=.61$

$K_{\mathrm{IC}}=$

$\mathrm{Gamma}=3.72$

2nd Technique $=$

Gamma $=$

$n=$

Environment $=$ air

Reference = ASHIZUKA,M.,BRADT,R., JACTAW, Vol. 65, No. 5, 1982

Comments $=$

Specific Material =

Manufacturer =

$\% \mathrm{Al}_{2} \mathrm{O}_{3}=$

$\% \mathrm{~B}_{2} \mathrm{O}_{3}=$

$\% \mathrm{~K}_{2} \mathrm{O}=$

$\% \mathrm{Na}_{2} \mathrm{O}=$

Other $\%=50$

$\% \mathrm{CaO}=$

$\% \mathrm{PbO}=$

Other Formula $=\mathrm{BaO}$

Other $\%=50$

$\% \mathrm{MgO}=$

$\% \mathrm{SiO}_{2}=$

Other Formula $=\mathrm{P}_{2} \mathrm{O}_{5}$

Young's Modulus $=4.32 \mathrm{E} 4$

1 st Technique $=$ Ind. Crk. Len.

$K_{\text {IC }}=.49$

2nd Technique $=$

$K_{\text {IC }}=$

Gamma $=2.78$

Gamma $=$

$n=$

Environment $=$ air

Reference $=$ ASHIZUKA,M.,BRADT,R., JACTAW, Vol. 65, No. 5, 1982

Comments $=$

Specific Material $=$

Manufacturer $=$

$\% \mathrm{Al}_{2} \mathrm{O}_{3}=$

$\% \mathrm{~B}_{2} \mathrm{O}_{3}=$

$\% \mathrm{~K}_{2} \mathrm{O}=$

$\% \mathrm{Na}_{2} \mathrm{O}=$

Other $\%=50$

$\% \mathrm{CaO}=$

$\% \mathrm{MgO}=50$

Other Formula $=\mathrm{P}_{2} \mathrm{O}_{5}$

Other $\%=$

$\% \mathrm{PbO}=$

$\% \mathrm{SiO}_{2}=$

Other Formula =

Young's Modulus $=5.4 E 4$

1 st Technique $=$ Ind. Crk. Len.

$K_{\mathrm{lC}}=.95$

2nd Technique $=$

$K_{\text {IC }}=$

Gamma $=8.36$

$n=$

Environment $=$ air

Reference = ASHIZUKA,M.,BRADT,R., JACTAW, Vol. 65, No., 5, 1982

Comments $=$ 
Specific Material =

Manufacturer $=$

$\% \mathrm{Al}_{2} \mathrm{O}_{3}=$

$\% \mathrm{Na}_{2} \mathrm{O}=$

Other Formula $=\mathrm{ZnO}$

$\% \mathrm{~B}_{2} \mathrm{O}_{3}=$

$\% \mathrm{~K}_{2} \mathrm{O}=$

Other $\%=50$

Other Formula $=\mathrm{P}_{2} \mathrm{O}_{5}$

Other $\%=50$
$\% \mathrm{CaO}=$

$\% \mathrm{PbO}=$

$K_{\text {IC }}=.6$

$K_{\text {IC }}=$
$\% \mathrm{MgO}=$

$\% \mathrm{SiO}_{2}=$

Young's Modulus $=4.24 E 4$

1st Technique $=$ Ind. Crk. Len.

2nd Technique $=$

$n=$

Environment $=$ air

Reference = ASHIZUKA,M.,BRADT,R., JACTAW, Vol. 65, No. 5, 1982

Comments $=$

Specific Material $=$

Manufacturer $=$

$\% \mathrm{Al}_{2} \mathrm{O}_{3}=$

$\% \mathrm{Na}_{2} \mathrm{O}=50$

$\% \mathrm{~B}_{2} \mathrm{O}_{3}=$

$\% \mathrm{~K}_{2} \mathrm{O}=$

Other Formula $=\mathrm{P}_{2} \mathrm{O}_{5}$

Other Formula =

Young's Modulus $=3.45 E 4$

1st Technique $=$ Ind. Crk. Len.

2nd Technique $=$

$n=$

Environment $=$ air

Reference = ASHIZUKA,M.,BRADT,R., JACTAW, Vol. 65, No. 5, 1982

Comments $=$

Specific Material =

Manufacturer =

$\% \mathrm{Al}_{2} \mathrm{O}_{3}=$

$\% \mathrm{Na}_{2} \mathrm{O}=$

$\mathrm{B}_{2} \mathrm{O}_{3}=$

$\% \mathrm{~K}_{2} \mathrm{O}=$

Other $\%=50$

Other Formula $=\mathrm{Li}_{2} \mathrm{O}$

Other $\%=50$

Other Formula $=\mathrm{P}_{2} \mathrm{O}_{5}$

Young's Modulus = $4.9 \mathrm{E} 4$

1st Technique $=$ Ind. Crk. Len.

2nd Technique $=$

$n=$

Environment $=$ air

Reference = ASHIZUKA,M.,BRADT,R., JACTAW, Vol. 65, No. 5, 1982

Comments $=$

\section{BRU77}

Specific Material $=$

Manufacturer $=$

$\% \mathrm{Al}_{2} \mathrm{O}_{3}=1.16$

$\% \mathrm{Na}_{2} \mathrm{O}=13.4$

Other Formula $=$

Other Formula =

Young's Modulus =

1st Technique $=\mathrm{DT}$

2nd Technique $=$

$n=$

Environment $=$ Toluene

Reference = BRUCE,J.,KOEPKE,B., JACTAW, Vol. 60, No. 5-6, 1977

Comments $=$
$\% \mathrm{~B}_{2} \mathrm{O}_{3}=$

$\% \mathrm{~K}_{2} \mathrm{O}=.632$

Other $\%=$

Other $\%=$
$K_{\mathrm{IC}}=.35$

$K_{\mathrm{IC}}=$

$\% \mathrm{CaO}=$

$\% \mathrm{PbO}=$

$K_{\mathrm{IC}}=.57$

$K_{\mathrm{IC}}=$

$\% \mathrm{CaO}=7.43$

$\% \mathrm{PbO}=$

$\% \mathrm{MgO}=5.91$

$\% \mathrm{SiO}_{2}=71.3$
$\% \mathrm{MgO}=$

$\% \mathrm{SiO}_{2}=$

Gamma $=3.32$

Gamma =

Gamma $=4.25$

Gamma =

$\% \mathrm{MgO}=$

$\% \mathrm{SiO}_{2}=$

Gamma $=1.78$

Gamma $=$

$$
\begin{array}{ll}
K_{\mathrm{IC}}=.77 & \text { Gamma }= \\
K_{\mathrm{IC}}= & \text { Gamma }=
\end{array}
$$


Specific Material =

Manufacturer $=$

$\% \mathrm{Al}_{2} \mathrm{O}_{3}=1.16$

$\% \mathrm{~B}_{2} \mathrm{O}_{3}=$

$\% \mathrm{~K}_{2} \mathrm{O}=.632$

Other \% =

Other Formula =

Other Formula =

Young's Modulus =

1st Technique $=\mathrm{DT}$

2nd Technique $=$

$n=$

Environment $=$ Mineral oil

Reference = BRUCE,J.,KOEPKE,B., JACTAW, Vol. 60, No. 5-6, 1977

Comments $=$

Specific Material $=$

Manufacturer $=$

$\% \mathrm{Al}_{2} \mathrm{O}_{3}=1.16$

$\% \mathrm{Na}_{2} \mathrm{O}=13.4$

Other Formula =

Other Formula $=$

Young's Modulus =

1st Technique $=\mathrm{DT}$

2nd Technique $=$

$n=$

Environment $=$ air

Reference $=$ BRUCE,J.,KOEPKE,B., JACTAW, Vol. 60, No. 5-6, 1977

Comments $=$

\section{CHA58}

Specific Material $=0080$

Manufacturer $=$ Corning Glass

$\% \mathrm{Al}_{2} \mathrm{O}_{3}=1.76$

$\% \mathrm{Na}_{2} \mathrm{O}=16.4$

Other Formula $=$

Other Formula $=$

Young's Modulus =
$\% \mathrm{~B}_{2} \mathrm{O}_{3}=$

$\% \mathrm{~K}_{2} \mathrm{O}=.632$

Other $\%=$

Other $\%=$
$\% \mathrm{CaO}=7.43$

$\% \mathrm{PbO}=$

$K_{\mathrm{IC}}=.77$

$K_{\text {IC }}=$
$\% \mathrm{MgO}=5.91$

$\% \mathrm{SiO}_{2}=71.3$ 1st Technique $=$ Dynamic Fat.

2nd Technique $=$
$\% \mathrm{~B}_{2} \mathrm{O}_{3}=$

$\% \mathrm{~K}_{2} \mathrm{O}=$

Other \% =

Other $\%=$
$\% \mathrm{CaO}=7.43$

$\% \mathrm{PbO}=$

$\% \mathrm{MgO}=5.91$

$\% \mathrm{SiO}_{2}=71.3$

$$
K_{\mathrm{IC}}=.76
$$$$
K_{\mathrm{IC}}=
$$

Gamma $=$ Gamma $=$

$\% \mathrm{CaO}=5.35$

$\% \mathrm{MgO}=4.46$

$\% \mathrm{PbO}=$

Gamma $=$

Gamma $=$

Gamma $=$

Gamma $=$

$n=16$

Environment $=$ air, $50 \% \mathrm{RH}$

Reference $=$ CHARLES,R.J., Jour. of Appl. Phys., 29, 1657-62, 1958

Comments $=$ Surface condition-abraded

\section{EAG78}

Specific Material =

Manufacturer $=$

\% $\mathrm{Al}_{2} \mathrm{O}_{3}=$

$\% \mathrm{Na}_{2} \mathrm{O}=40$

Other Formula $=$

Other Formula =

Young's Modulus $=6.0 E 4$
$\% \mathrm{~B}_{2} \mathrm{O}_{3}=$
$\% \mathrm{~K}_{2} \mathrm{O}=$
Other $\%=$
Other $\%=$

1st Technique $=\mathrm{NB}$

2nd Technique $=$

$K_{\text {IC }}=$

$K_{\text {IC }}=$

$n=$

Environment $=$ air

Reference = EAGAN,R.,SWEARENGEN,J.JACTAW, Vol 61, No 1-2, 27-30, 1978

Comments $=$

$$
\begin{array}{ll}
K_{\mathrm{IC}}=.62 & \text { Gamma }=3.15 \\
K_{\mathrm{IC}}= & \text { Gamma }=
\end{array}
$$

$$
\begin{array}{ll}
\% \mathrm{CaO}= & \% \mathrm{MgO}= \\
\% \mathrm{PbO}= & \% \mathrm{SiO}_{2}=60
\end{array}
$$


Specific Material = Manufacturer $=$
$\% \mathrm{Al}_{2} \mathrm{O}_{3}=8$
$\% \mathrm{~B}_{2} \mathrm{O}_{3}=$
$\% \mathrm{CaO}=$
$\% \mathrm{MgO}=$
$\% \mathrm{Na}_{2} \mathrm{O}=32$
Other Formula =
$\% \mathrm{~K}_{2} \mathrm{O}=$
$\% \mathrm{PbO}=$
$\% \mathrm{SiO}_{2}=60$
Other \% =
Other $\%=$
Other Formula =
Young's Modulus $=6.9 E 4$
1st Technique $=\mathrm{NB}$
$K_{\mathrm{IC}}=.64$
Gamma $=2.97$
2nd Technique $=$
$K_{\mathrm{IC}}=$
Gamma $=$

$n=$

Environment $=$ air

Reference = EAGAN,R.,SWEARENGEN,J.,JACTAW, Vol 61, No 1-2, 27-30, 1978

Comments $=$

Specific Material =

Manufacturer $=$

$\% \mathrm{Al}_{2} \mathrm{O}_{3}=16.2$

$\% \quad \mathrm{Na}_{2} \mathrm{O}=23.8$

Other Formula $=$

$\% \mathrm{~B}_{2} \mathrm{O}_{3}=$

$\% \mathrm{~K}_{2} \mathrm{O}=$

Other $\%=$

Other $\%=$
$\% \mathrm{CaO}=$

$\% \mathrm{PbO}=$

$K_{\mathrm{IC}}=.62$

$K_{\mathrm{IC}}=$
$\% \mathrm{MgO}=$

$\% \mathrm{SiO}_{2}=60$

Other Formula =

Young's Modulus $=7.25 E 4$

Ist Technique $=\mathrm{NB}$

2nd Technique $=$

$n=$

Environment $=$ air

Reference = EAGAN,R.,SWEARENGEN,J.,JACTAW, Vol 61, No 1-2, 27-30, 1978

Comments $=$

Specific Material =

Manufacturer $=$

$\% \mathrm{Al}_{2} \mathrm{O}_{3}=20$

$\% \mathrm{Na}_{2} \mathrm{O}=20$

$\% \mathrm{~B}_{2} \mathrm{O}_{3}=$

$\% \mathrm{~K}_{2} \mathrm{O}=$

Other $\%=$

Other Formula =

Other Formula =

Young's Modulus $=7.3 E 4$

1st Technique $=\mathrm{NB}$

2nd Technique $=$

$n=$

Environment $=$ air

Reference = EAGAN,R.,SWEARENGEN,J.,JACTAW, Vol 61, No 1-2, 27-30, 1978

Comments $=$

Specific Material $=$

Manufacturer $=$

$\% \mathrm{Al}_{2} \mathrm{O}_{3}=22.8$

$\% \mathrm{Na}_{2} \mathrm{O}=17.2$

Other Formula =

Other Formula =

Young's Modulus $=7.8 E 4$

1 st Technique $=\mathrm{NB}$

2nd Technique $=$

Other $\%=$
$\% \mathrm{CaO}=$

$\% \mathrm{PbO}=$

$K_{\mathrm{IC}}=.67$

$K_{\mathrm{IC}}=$
$K_{\text {IC }}=.70$

$K_{\mathrm{IC}}=$
$\% \mathrm{CaO}=$

$\% \mathrm{PbO}=$

Other $\%=$

Other $\%=$
Gamma $=2.61$

Gamma $=$

$n=$

Environment $=$ air

Reference = EAGAN,R.,SWEARENGEN,J.,JACTAW, Vol 61, No 1-2, 27-30, 1978

Comments $=$

$\% \mathrm{MgO}=$

$\% \mathrm{SiO}_{2}=60$

Gamma $=3.10$

Gamma $=$ 
Specific Material $=$

Manufacturer $=$

$\% \mathrm{Al}_{2} \mathrm{O}_{3}=$

$\% \mathrm{Na}_{2} \mathrm{O}=20$

Other Formula =

$\% \mathrm{~B}_{2} \mathrm{O}_{3}=$

$\% \mathrm{~K}_{2} \mathrm{O}=$

Other \% =

Other $\%=$

Other Formula =

Young's Modulus $=6.1 E 4$

1st Technique $=\mathrm{NB}$

2nd Technique $=$

$K_{\mathrm{IC}}=.58$

$\% \mathrm{CaO}=$

$\% \mathrm{MgO}=$

$\% \mathrm{PbO}=$

$\% \mathrm{SiO}_{2}=80$

$n=$

$K_{\mathrm{IC}}=$

Gamma $=2.76$

Environment $=$ air

Reference = EAGAN,R.,SWEARENGEN,J.,JACTAW, Vol 61, No 1-2, 27-30, 1978

Comments $=$

Specific Material $=$

Manufacturer $=$

$\% \mathrm{Al}_{2} \mathrm{O}_{3}=5$

$\% \mathrm{~B}_{2} \mathrm{O}_{3}=$

$\% \mathrm{Na}_{2} \mathrm{O}=20$

Other Formula =

$\% \mathrm{~K}_{2} \mathrm{O}=$

Other \% =

Other Formula =

Other $\%=$

Gamma =

Young's Modulus $=6.4 E 4$

1 st Technique $=\mathrm{NB}$

2nd Technique $=$

$K_{\mathrm{IC}}=.63$

$\% \mathrm{CaO}=$

$\% \mathrm{MgO}=$

$\% \mathrm{PbO}=$

$\% \mathrm{SiO}_{2}=75$

$n=$

Environment $=$ air

Reference = EAGAN,R.,SWEARENGEN,J.,JACTAW, Vol 61, No 1-2, 27-30, 1978

Comments $=$

Specific Material $=$

Manufacturer $=$

$\% \mathrm{Al}_{2} \mathrm{O}_{3}=10$

$\% \mathrm{Na}_{2} \mathrm{O}=20$

Other Formula $=$

Other Formula =

Young's Modulus $=6.9 E 4$

1 st Technique $=\mathrm{NB}$

2nd Technique $=$

$K_{\mathrm{IC}}=$

Gamma $=3.10$

Gamma $=$

$n=$

Environment $=$ air

Reference = EAGAN,R.,SWEARENGEN,J.,JACTAW, Vol 61, No 1-2, 27-30, 1978

Comments $=$

Specific Material $=$

Manufacturer $=$

$\% \mathrm{Al}_{2} \mathrm{O}_{3}=20$

$\% \mathrm{~B}_{2} \mathrm{O}_{3}=$

$\% \mathrm{~K}_{2} \mathrm{O}=$

$\% \mathrm{CaO}=$

$\% \mathrm{MgO}=$

Other \% =

$\% \mathrm{PbO}=$

$\% \mathrm{SiO}_{2}=70$

$\% \mathrm{Na}_{2} \mathrm{O}=20$

Other Formula =

Other Formula =

Young's Modulus = 7.25 E4

Other $\%=$

$$
\begin{array}{ll}
K_{\mathrm{IC}}=.67 & \text { Gamma }=3.25 \\
K_{\mathrm{IC}}= & \text { Gamma }=
\end{array}
$$

1st Technique $=\mathrm{NB}$

2nd Technique $=$

$n=$

Environment $=$ air

Reference = EAGAN,R.,SWEARENGEN,J.,JACTAW, Vol 61, No 1-2, 27-30, 1978

Comments $=$

$$
\begin{array}{ll}
K_{\mathrm{IC}}=.67 & \text { Gamma }=3.10 \\
K_{\mathrm{IC}}= & \text { Gamma }=
\end{array}
$$

$$
\begin{array}{ll}
\% \mathrm{CaO}= & \% \mathrm{MgO}= \\
\% \mathrm{PbO}= & \% \mathrm{SiO}_{2}=60
\end{array}
$$

Other $\%=$

Other $\%=$ 
Specific Material $=$

Manufacturer $=$

$\% \mathrm{Al}_{2} \mathrm{O}_{3}=24$

$\% \mathrm{~B}_{2} \mathrm{O}_{3}=$

$\% \mathrm{~K}_{2} \mathrm{O}=$

Other $\%=$

Other Formula =

Other Formula =

Young's Modulus $=7.75 E 4$

1st Technique $=\mathrm{NB}$

2nd Technique $=$

$n=$

Environment $=$ air

Reference = EAGAN,R.,SWEARENGEN,J.JACTAW, Vol 61, No 1-2, 27-30, 1978

Comments $=$

Specific Material =

Manufacturer =

$\% \quad \mathrm{Al}_{2} \mathrm{O}_{3}=32$

$\% \mathrm{Na}_{2} \mathrm{O}=$

Other Formula =

Other Formula =

Young's Modulus $=9.3 E 4$

lst Technique $=\mathrm{NB}$

2nd Technique $=$

$n=$

Environment $=$ air

Reference = EAGAN,R.,SWEARENGEN,J.,JACTAW, Vol 61, No 1-2, 27-30, 1978

Comments $=$

Specific Material =

Manufacturer $=$

$\% \mathrm{Al}_{2} \mathrm{O}_{3}=13.3$

$\% \mathrm{Na}_{2} \mathrm{O}=$

Other Formula =

Other Formula =

Young's Modulus $=9.2 E 4$

1st Technique $=\mathrm{NB}$

2nd Technique $=$
$\% \mathrm{~B}_{2} \mathrm{O}_{3}=$

$\% \mathrm{~K}_{2} \mathrm{O}=$

Other \% =

Other $\%=$
$\% \mathrm{CaO}=$

$\% \mathrm{PbO}=$

$K_{\text {IC }}=.67$

$K_{\text {IC }}=$
Gamma $=2.85$

Gamma =
$\% \mathrm{SiO}_{2}=56$
$\% \mathrm{MgO}=$
$\% \mathrm{~B}_{2} \mathrm{O}_{3}=$

$\% \mathrm{~K}_{2} \mathrm{O}=$

Other \% =

Other $\%=$
$K_{\text {IC }}=.6$

$K_{\mathrm{lC}}=$

$\% \mathrm{CaO}=8$

$\% \mathrm{PbO}=$

$\% \mathrm{MgO}=$

$\% \mathrm{SiO}_{2}=60$

Gamma $=$
$K_{\mathrm{IC}}=.65$

$\% \mathrm{CaO}=26.7$

$\% \mathrm{PbO}=$

Gamma $=1.94$

Gamma $=2.26$

Gamma =

$n=$

Environment $=$ air

Reference = EAGAN,R.,SWEARENGEN,J.JACTAW, Vol 61, No 1-2, 27-30, 1978

Comments $=$

Specific Material $=$

Manufacturer =

$\% \mathrm{Al}_{2} \mathrm{O}_{3}=20$

$\% \mathrm{Na}_{2} \mathrm{O}=$

Other Formula =

Other Formula $=$

Young's Modulus $=9.35 E 4$

1st Technique $=$ NB

2nd Technique $=$

$n=$

Environment $=$ air

Reference = EAGAN,R.,SWEARENGEN,J.,JACTAW, Vol 61, No 1-2, 27-30, 1978

Comments $=$

$K_{\text {IC }}=.66$

$K_{\mathrm{IC}}=$

$\% \mathrm{CaO}=20$

$\% \mathrm{PbO}=$

\% $\mathrm{MgO}=$

$\% \mathrm{SiO}_{2}=60$

Other $\%=$

Other $\%=$ 
Specific Material =

Manufacturer $=$

$\% \mathrm{Al}_{2} \mathrm{O}_{3}=22.8$

$\% \mathrm{~B}_{2} \mathrm{O}_{3}=$

$\% \mathrm{~K}_{2} \mathrm{O}=$

Other \% =

Other $\%=$

Other Formula =

Other Formula =

Young's Modulus $=9.8 E 4$

1st Technique $=\mathrm{NB}$

2nd Technique $=$

$n=$

Environment $=$ air

Reference = EAGAN,R.,SWEARENGEN,J.,JACTAW, Vol 61, No 1-2, 27-30, 1978

Comments $=$

Specific Material $=$

Manufacturer =

$\% \mathrm{Al}_{2} \mathrm{O}_{3}=$

$\% \mathrm{Na}_{2} \mathrm{O}=40$

$\% \mathrm{~B}_{2} \mathrm{O}_{3}=$

$\% \mathrm{~K}_{2} \mathrm{O}=$

Other \% =

Other $\%=$
$\% \mathrm{CaO}=17.2$

$\% \mathrm{PbO}=$

$K_{\mathrm{IC}}=.66$

$K_{\mathrm{IC}}=$
$\% \mathrm{MgO}=$

$\% \mathrm{SiO}_{2}=60$

Other Formula $=$

Young's Modulus $=6.1 E 4$

1 st Technique $=\mathrm{NB}$

2nd Technique $=$

$n=$

Environment $=$ air

Reference = EAGAN,R.,SWEARENGEN,J.,JACTAW, Vol 61, No 1-2, 27-30, 1978

Comments $=$

Specific Material $=$

Manufacturer =

$\% \mathrm{Al}_{2} \mathrm{O}_{3}=$

$\% \mathrm{Na}_{2} \mathrm{O}=32$

Other Formula =

Other Formula =

Young's Modulus $=6.5 E 4$

1st Technique $=\mathrm{NB}$

2nd Technique $=$

$\% \mathrm{CaO}=$

$\% \mathrm{PbO}=$

$K_{\mathrm{IC}}=.63$

$K_{\text {IC }}=$

Gamma $=2.19$

Gamma $=$

$n=$

Environment $=$ air

Reference = EAGAN,R.,SWEARENGEN,J.,JACTAW, Vol 61, No 1-2, 27-30, 1978

Comments $=$

Specific Material =

Manufacturer =

$\% \mathrm{Al}_{2} \mathrm{O}_{3}=$

$\% \mathrm{Na}_{2} \mathrm{O}=23.8$

Other Formula =

$\% \mathrm{~B}_{2} \mathrm{O}_{3}=8$

$\% \mathrm{~K}_{2} \mathrm{O}=$

Other \% =

Other $\%=$

$$
\begin{array}{ll}
\% \mathrm{CaO}= & \% \mathrm{MgO}= \\
\% \mathrm{PbO}= & \% \mathrm{SiO}_{2}=60
\end{array}
$$

$K_{\text {IC }}=.75$

$K_{\mathrm{IC}}=$

Gamma $=4.33$

Gamma =

Other Formula =

Young's Modulus $=8.4 \mathrm{E} 4$

1st Technique $=\mathrm{NB}$

2nd Technique $=$

$$
\begin{array}{ll}
\% & \mathrm{~B}_{2} \mathrm{O}_{3}=16.2 \\
\% & \mathrm{~K}_{2} \mathrm{O}= \\
& \text { Other \%= } \\
& \text { Other \% }=
\end{array}
$$

$\% \mathrm{CaO}=$

$\% \mathrm{PbO}=$
Gamma $=3.20$

Gamma $=$

$n=$

Environment $=$ air

Reference = EAGAN,R.,SWEARENGEN,J.,JACTAW, Vol 61, No 1-2, 27-30, 1978

Comments $=$

$K_{\mathrm{IC}}=.94$

$K_{\mathrm{IC}}=$
Gamma $=5.26$

Gamma $=$ 
Specific Material =

Manufacturer $=$

$\% \mathrm{Al}_{2} \mathrm{O}_{3}=$

$\% \mathrm{Na}_{2} \mathrm{O}=20$

Other Formula =

$\% \mathrm{~B}_{2} \mathrm{O}_{3}=20$

$\% \mathrm{~K}_{2} \mathrm{O}=$

Other $\%=$

Other $\%=$

$$
\% \mathrm{CaO}=
$$

$\% \mathrm{PbO}=$

$\% \mathrm{MgO}=$

$\% \mathrm{SiO}_{2}=60$

Other Formula =

Young's Modulus $=8.2 E 4$

1st Technique $=\mathrm{NB}$

2nd Technique $=$

$K_{\text {IC }}=.88$

$K_{\mathrm{IC}}=$

Gamma $=4.72$

$n=$

Environment $=$ air

Reference = EAGAN,R.,SWEARENGEN,J.,JACTAW, Vol 61, No 1-2, 27-30, 1978

Comments $=$

Specific Material =

Manufacturer $=$

$\% \mathrm{Al}_{2} \mathrm{O}_{3}=$

$\% \mathrm{Na}_{2} \mathrm{O}=17.2$

Other Formula $=$

Other Formula $=$

Young's Modulus $=8.6 E 4$

1st Technique $=\mathrm{NB}$

2nd Technique $=$

$\% \mathrm{~B}_{2} \mathrm{O}_{3}=22.8$

$\% \mathrm{~K}_{2} \mathrm{O}=$

Other $\%=$

Other $\%=$
Gamma $=$

$n=$

Environment $=$ air

Reference = EAGAN,R.,SWEARENGEN,J.,JACTAW, Vol 61, No 1-2, 27-30, 1978

Comments $=$

\section{FRE83}

Specific Material =

Manufacturer $=$

$\% \mathrm{Al}_{2} \mathrm{O}_{3}=$

$\% \mathrm{Na}_{2} \mathrm{O}=$

Other Formula $=\mathrm{Li}_{2} \mathrm{O}$

Other Formula =

Young's Modulus =

1st Technique $=\mathrm{DCB}$

2nd Technique $=$

$n=11$

Environment $=\mathrm{H}_{2} \mathrm{O}$

Reference $=$ FREIMAN,S.W., Private Communications, 1983

Comments $=$

Specific Material = Low Iron Float

Manufacturer $=$

$\% \mathrm{Al}_{2} \mathrm{O}_{3}=1.16$

$\% \mathrm{Na}_{2} \mathrm{O}=13.4$

Other Formula $=$

Other Formula $=$

Young's Modulus =

1st Technique $=\mathrm{DCB}$

2nd Technique $=$

$n=19.3$

Environment $=\mathrm{H}_{2} \mathrm{O}$

Reference $=$ FREIMAN, S.W., et al, Submitted to JACTAW, 1983

Comments $=$
$\% \mathrm{~B}_{2} \mathrm{O}_{3}=$

$\% \mathrm{~K}_{2} \mathrm{O}=$

Other $\%=33$

Other $\%=$
Other $\%=$

Other $\%=$
$\% \mathrm{CaO}=$

$\% \mathrm{PbO}=$

$\% \mathrm{MgO}=$

$\% \mathrm{SiO}_{2}=60$

$$
K_{\mathrm{IC}}=.92
$$$$
K_{\mathrm{IC}}=
$$

Gamma $=4.89$

Gamma $=$

$$
\begin{array}{ll}
K_{\mathrm{IC}}= & \text { Gamma }= \\
K_{\mathrm{IC}}= & \text { Gamma }=
\end{array}
$$
$\% \mathrm{CaO}=$
$\% \mathrm{PbO}=$
$\% \mathrm{MgO}=$
$\% \mathrm{SiO}_{2}=67$
$\% \mathrm{~B}_{2} \mathrm{O}_{3}=$
$\% \mathrm{~K}_{2} \mathrm{O}=.632$
$\% \mathrm{CaO}=7.43$
$\% \mathrm{MgO}=5.91$
$\% \mathrm{PbO}=$
$\% \mathrm{SiO}_{2}=71.3$

$$
\begin{array}{ll}
K_{\mathrm{lC}}= & \text { Gamma }= \\
K_{\mathrm{lC}}= & \text { Gamma }=
\end{array}
$$


Specific Material $=$ Low Iron Float

Manufacturer $=$

$\% \mathrm{Al}_{2} \mathrm{O}_{3}=1.16$

$\% \mathrm{~B}_{2} \mathrm{O}_{3}=$

$\% \mathrm{Na}_{2} \mathrm{O}=13.4$

$\% \mathrm{~K}_{2} \mathrm{O}=.632$

$\% \mathrm{CaO}=7.43$

$\% \mathrm{MgO}=5.91$

Other Formula =

Other $\%=$

$\% \mathrm{PbO}=$

$\% \mathrm{SiO}_{2}=71.3$

Other Formula =

Other $\%=$

Young's Modulus =

1st Technique $=$ Dynamic Fat.

$K_{\mathrm{IC}}=$

Gamma =

2nd Technique $=$

$K_{\mathrm{IC}}=$

Gamma $=$

$n=15.2$

Environment $=\mathrm{H}_{2} \mathrm{O}$

Reference = FREIMAN, S.W., et al, Submitted to JACTAW, 1983

Comments $=$

Specific Material $=7809$

Manufacturer $=$ Corning Glass

$\% \mathrm{Al}_{2} \mathrm{O}_{3}=5.72 \quad \% \mathrm{~B}_{2} \mathrm{O}_{3}=7.45$

$\% \mathrm{~K}_{2} \mathrm{O}=3.44$

$\% \mathrm{CaO}=2.31$

$\% \mathrm{MgO}=9.41$

$\% \mathrm{Na}_{2} \mathrm{O}=9.41$

Other $\%=.405$

$\% \mathrm{PbO}=$

$\% \mathrm{SiO}_{2}=71.2$

Other Formula $=\mathrm{TiO}_{2}$

Other $\%=<.1 \mathrm{~W} \%$

Other Formula $=\mathrm{Fe}_{2} \mathrm{O}_{3}$

$\%$ Young's Modulus =

1st Technique $=\mathrm{DCB}$

2nd Technique $=$

$n=25.3$

Environment $=\mathrm{H}_{2} \mathrm{O}$

Reference = FREIMAN, S.W., et al, Submitted to JACTAW, 1983

Comments $=$

Specific Material $=7809$

Manufacturer $=$ Corning Glass

$\% \mathrm{Al}_{2} \mathrm{O}_{3}=5.72 \quad \% \mathrm{~B}_{2} \mathrm{O}_{3}=7.45$

$\% \mathrm{~K}_{2} \mathrm{O}=3.44$

$\% \mathrm{CaO}=2.31$

$\% \mathrm{MgO}=9.41$

$\% \mathrm{Na}_{2} \mathrm{O}=9.41$

Other $\%=.405$

$\% \mathrm{PbO}=$

$\% \mathrm{SiO}_{2}=71.2$

Other Formula $=\mathrm{TiO}_{2}$

Other $\%=<.1 \mathrm{~W} \%$

Other Formula $=\mathrm{Fe}_{2} \mathrm{O}_{3}$

Young's Modulus =

1st Technique $=$ Dynamic Fat

2nd Technique $=$

$n=31.4$

$K_{\mathrm{IC}}=$

$K_{\mathrm{IC}}=$

Gamma $=$

Gamma $=$

Environment $=\mathrm{H}_{2} \mathrm{O}$

Reference = FREIMAN, S.W., et al, Submitted to JACTAW, 1983

Comments $=$

Specific Material $=0317$

Manufacturer $=$ Corning Glass

$\% \mathrm{Al}_{2} \mathrm{O}_{3}=18.8$

$\% \mathrm{~B}_{2} \mathrm{O}_{3}=8.08$

$\% \mathrm{Na}_{2} \mathrm{O}=$

$\% \mathrm{~K}_{2} \mathrm{O}=$

$\% \mathrm{CaO}=$

$\% \mathrm{MgO}=$

Other Formula =

Other $\%=$

$\% \mathrm{PbO}=$

$\% \mathrm{SiO}_{2}=73.0$

Other Formula =

Young's Modulus =

Other $\%=$

1st Technique $=$ DCB

2nd Technique $=$

$n=25.1$

$K_{\mathrm{IC}}=$

Gamma $=$

$K_{\mathrm{IC}}=$

Gamma $=$

Environment $=\mathrm{H}_{2} \mathrm{O}$

Reference = FREIMAN, S.W., et al, Submitted to JACTAW, 1983

Comments $=\%$ composition $-9.5 \%$ modifiers 
Specific Material $=0317$

Manufacturer $=$ Corning Glass
$\% \mathrm{Al}_{2} \mathrm{O}_{3}=18.8$
$\% \mathrm{~B}_{2} \mathrm{O}_{3}=8.08$
$\% \mathrm{Na}_{2} \mathrm{O}=$
$\% \mathrm{~K}_{2} \mathrm{O}=$
Other Formula =
Other $\%=$
Other Formula =
Other $\%=$

$\% \mathrm{CaO}=$

$\% \mathrm{MgO}=$

$\% \mathrm{PbO}=$

$\% \mathrm{SiO}_{2}=73.0$

Young's Modulus =

1st Technique $=$ Dynamic Fat.

$K_{\mathrm{IC}}=$

$K_{\mathrm{IC}}=$

Gamma =

2nd Technique $=$

$n=32.6$

Environment $=\mathrm{H}_{2} \mathrm{O}$

Reference $=$ FREIMAN, S.W., et al, Submitted to JACTAW, 1983

Comments $=\%$ composition $-9.5 \%$ modifier

\section{FRE84}

Specific Material $=8244$

Manufacturer $=$ NBS

$\% \mathrm{Al}_{2} \mathrm{O}_{3}=\quad \% \mathrm{~B}_{2} \mathrm{O}_{3 .}=$

$\% \mathrm{Na}_{2} \mathrm{O}=\quad \% \mathrm{~K}_{2} \mathrm{O}=$

Other Formula $=\mathrm{P}_{2} \mathrm{O}_{5} \quad$ Other $\%=74.6$

Other Formula $=\mathrm{La}_{2} \mathrm{O}_{3} \quad$ Other $\%=25$

$\% \mathrm{CaO}=$

$\% \mathrm{PbO}=$

$\% \mathrm{MgO}=$

$\% \mathrm{SiO}_{2}=$

Young's Modulus $=6.05 E 4$

1 st Technique $=$ Ind. Crk. Len.

$K_{\mathrm{IC}}=.58$

2nd Technique $=$

$K_{\text {IC }}=$

Gamma $=2.78$

$n=$

Environment $=$ Heptane

Reference $=$ FREIMAN,S.W., Private Communications, 1984

Comments $=3$ rd Other Oxide- $0.5 \% \quad \mathrm{Nd}_{2} \mathrm{O}_{3}$, \& Young's Modulus is estimated

Specific Material $=8245$

Manufacturer $=$ NBS

$\% \mathrm{Al}_{2} \mathrm{O}_{3}=25$

$\% \mathrm{Na}_{2} \mathrm{O}=$

$\% \mathrm{~B}_{2} \mathrm{O}_{3}=$

$\% \mathrm{~K}_{2} \mathrm{O}=$

Other Formula $=\mathrm{P}_{2} \mathrm{O}_{5} \quad$ Other $\%=74.6$

Other Formula $=\mathrm{Nd}_{2} \mathrm{O}_{3} \quad$ Other $\%=0.5$

Young's Modulus $=8.7 E 4$

1st Technique $=$ Ind. Crk. Len.

2nd Technique $=$

$n=$

Environment $=$ Heptane

Reference $=$ FREIMAN,S.W., Private Communications, 1984

Comments $=$ Young's Modulus is estimated

Specific Material $=9023$

Manufacturer $=\mathrm{GTE}$

$\% \mathrm{Al}_{2} \mathrm{O}_{3}=25$

$\% \mathrm{Na}_{2} \mathrm{O}=$

$\% \mathrm{~B}_{2} \mathrm{O}_{3}=$

$\% \mathrm{~K}_{2} \mathrm{O}=$

Other Formula $=\mathrm{P}_{2} \mathrm{O}_{5} \quad$ Other $\%=74.6$

$K_{\text {IC }}=.58$

$K_{\mathrm{lc}}=$

$\% \mathrm{CaO}=$

$\% \mathrm{PbO}=$

$\% \mathrm{MgO}=$

$\% \mathrm{SiO}_{2}=$

$\% \mathrm{CaO}=$

$\% \mathrm{MgO}=$

$\% \mathrm{PbO}=$

Gamma $=$

Other Formula $=\mathrm{Nd}_{2} \mathrm{O}_{3} \quad$ Other $\%=0.4$

Young's Modulus $=8.7 \mathrm{E} 4$

1st Technique $=$ Ind. Crk. Len.

2nd Technique $=$

$K_{\mathrm{IC}}=.72$

Gamma $=1.93$

Gamma $=2.98$

Gamma $=$

$n=$

Environment $=$ Heptane

Reference $=$ FREIMAN,S.W., Private Communications, 1984

Comments $=$ Young's Modulus is estimated 
Specific Material $=$ UP63-6771

Manufacturer $=$

$\% \mathrm{Al}_{2} \mathrm{O}_{3}=$

$\% \mathrm{Na}_{2} \mathrm{O}=$

Other Formula $=\mathrm{P}_{2} \mathrm{O}_{5}$

Other Formula =

Young's Modulus $=4.67 E 4$

$\% \mathrm{~B}_{2} \mathrm{O}_{3}=$

$\% \mathrm{~K}_{2} \mathrm{O}=$

Other $\%=70$

Other $\%=$

1st Technique $=$ Ind. Crk. Len.

2nd Technique $=$

$n=$

Environment $=\mathrm{N}_{2}(\mathrm{~g})$

Reference $=$ FREIMAN,S.W., Private Communications, 1984

Comments $=$ Compo.- $+10 \%\left(\mathrm{La}_{2} \mathrm{O}_{3}+\mathrm{Nd}_{2} \mathrm{O}_{3}\right)$

Specific Material = UP37-6830

Manufacturer =
$\% \mathrm{Al}_{2} \mathrm{O}_{3}=$
$\% \mathrm{~B}_{2} \mathrm{O}_{3}=$
$\% \mathrm{Na}_{2} \mathrm{O}=$
$\% \mathrm{~K}_{2} \mathrm{O}=12$
Other Formula $=\mathrm{P}_{2} \mathrm{O}_{5}$
Other $\%=65$
Other Formula =
Other $\%=$

$$
\begin{aligned}
& K_{\mathrm{IC}}=.33 \\
& K_{\mathrm{IC}}=
\end{aligned}
$$

$\% \mathrm{MgO}=20$

$\% \mathrm{SiO}_{2}=$

Gamma $=1.17$

Gamma =

Gamma $=1.14$

$$
\begin{aligned}
& \% \mathrm{CaO}= \\
& \% \mathrm{PbO}=
\end{aligned}
$$

$K_{\mathrm{IC}}=.35$

$K_{\text {IC }}=$
Gamma $=$

$\% \mathrm{SiO}_{2}=$

$\% \mathrm{CaO}=$

$\% \mathrm{PbO}=$

$K_{\text {IC }}=.33$

$K_{\mathrm{IC}}=$

Gamma $=$

$\% \mathrm{MgO}=$

$\% \mathrm{SiO}_{2}=$

Gamma $=1.23$

$\begin{array}{ll}\text { Other Formula }=\mathrm{P}_{2} \mathrm{O}_{5} & \text { Other } \%=62 \\ \text { Other Formula }= & \text { Other } \%=\end{array}$

Young's Modulus $=4.42 E 4$

1 st Technique $=$ Ind. Crk. Len.

2nd Technique $=$

$n=$

Environment $=\mathrm{N}_{2}(\mathrm{~g})$

Reference $=$ FREIMAN,S.W., Private Communications, 1984

Comments $=$ Compo. $-+13 \%\left(\mathrm{La}_{2} \mathrm{O}_{3}+\mathrm{Nd}_{2} \mathrm{O}_{3}\right)$

Specific Material $=$ MJ-4-5288

Manufacturer $=$

$\% \mathrm{Al}_{2} \mathrm{O}_{3}=15$

$\% \mathrm{Na}_{2} \mathrm{O}=$

$\% \mathrm{~B}_{2} \mathrm{O}_{3}=$

$\% \mathrm{~K}_{2} \mathrm{O}=$

Other Formula $=\mathrm{P}_{2} \mathrm{O}_{5}$

Other $\%=69$

Other Formula =

Other $\%=$

Young's Modulus $=6.72 E 4$

1st Technique $=$ Ind. Crk. Len.

2nd Technique $=$

$K_{\mathrm{IC}}=.55$

$\% \mathrm{CaO}=$

$\% \mathrm{PbO}=$

$\% \mathrm{MgO}=15$

$\% \mathrm{SiO}_{2}=$

$n=$

$K_{\mathrm{IC}}=$

Gamma $=2.25$

Gamma $=$

Environment $=\mathrm{N}_{2}(\mathrm{~g})$

Reference $=$ FREIMAN,S.W., Private Communications, 1984

Comments $=$ Compo.- $+2 \%\left(\mathrm{La}_{2} \mathrm{O}_{3}+\mathrm{Nd}_{2} \mathrm{O}_{3}\right)$ 
Specific Material = UP86-6961

Manufacturer $=$

$\% \mathrm{Al}_{2} \mathrm{O}_{3}=9.4$

$\% \mathrm{~B}_{2} \mathrm{O}_{3}=$

$\% \mathrm{Na}_{2} \mathrm{O}=$

$\% \mathrm{~K}_{2} \mathrm{O}=9.4$

$\% \mathrm{CaO}=$

$\% \mathrm{MgO}=$

Other Formula $=\mathrm{P}_{2} \mathrm{O}_{5}$

Other $\%=75.3$

$\% \mathrm{PbO}=$

$\% \mathrm{SiO}_{2}=$

Other Formula $=\mathrm{La}_{2} \mathrm{O}_{3} \quad$ Other $\%=1.6$

Young's Modulus $=6.37 E 4$

1st Technique $=$ Ind. Crk. Len.

$K_{\mathrm{IC}}=.37$

$K_{\mathrm{IC}}=$

Gamma $=1.08$

2nd Technique $=$

Gamma $=$

$n=$

Environment $=$ Heptane

Reference = FREIMAN,S.W., Private Communications, 1984

Comments = Compo.- 3 rd Other Oxide- $4.3 \% \quad \mathrm{Nd}_{2} \mathrm{O}_{3}$

Specific Material $=$ UP77-6945

Manufacturer $=$

$\% \mathrm{Al}_{2} \mathrm{O}_{3}=16.5$

$\% \mathrm{Na}_{2} \mathrm{O}=$

$\% \mathrm{~B}_{2} \mathrm{O}_{3}=$

$\% \mathrm{~K}_{2} \mathrm{O}=12.5$

Other Formula $=\mathrm{P}_{2} \mathrm{O}_{5} \quad$ Other $\%=65.1$

Other Formula $=\mathrm{La}_{2} \mathrm{O}_{3} \quad$ Other $\%=1.7$

Young's Modulus $=6.55 E 4$

1st Technique $=$ Ind. Crk. Len.

2nd Technique $=$

$K_{\mathrm{IC}}=.45$

$\% \mathrm{CaO}=$

$\% \mathrm{PbO}=$

$\% \mathrm{MgO}=$

$\% \mathrm{SiO}_{2}=$

$n=$

Environment $=$ Heptane

Reference $=$ FREIMAN,S.W., Private Communications, 1984

Comments $=$ Compo.- 3 rd Other Oxide- $4.2 \% \quad \mathrm{Nd}_{2} \mathrm{O}_{3}$

Specific Material = UP69-6897

Manufacturer =

$\% \mathrm{Al}_{2} \mathrm{O}_{3}=11.8$

$\% \mathrm{Na}_{2} \mathrm{O}=$

$\% \mathrm{~B}_{2} \mathrm{O}_{3}=$

$\% \mathrm{~K}_{2} \mathrm{O}=23.6$

Other Formula $=\mathrm{P}_{2} \mathrm{O}_{5} \quad$ Other $\%=59$

Other Formula $=\mathrm{La}_{2} \mathrm{O}_{3} \quad$ Other $\%=1.5$

Gamma $=1.55$

Gamma $=$

Young's Modulus $=5.37 E 4$

1st Technique $=$ Ind. Crk. Len.

$K_{\mathrm{IC}}=.33$

$\% \mathrm{CaO}=$

$\% \mathrm{PbO}=$

$\% \mathrm{MgO}=$

$\% \mathrm{SiO}_{2}=$

2nd Technique $=$

$K_{\mathrm{IC}}=$

Gamma $=1.01$

$n=$

Environment $=$ Heptane

Reference = FREIMAN,S.W., Private Communications, 1984

Comments $=$ Compo.- 3 rd Other Oxide- $4.1 \% \quad \mathrm{Nd}_{2} \mathrm{O}_{3}$

Specific Material $=$ UP70-6937

Manufacturer $=$

$\% \mathrm{Al}_{2} \mathrm{O}_{3}=15.9$

$\% \cdot \mathrm{Na}_{2} \mathrm{O}=$

$\% \mathrm{~B}_{2} \mathrm{O}_{3}=$

$\% \mathrm{~K}_{2} \mathrm{O}=15.9$

Other Formula $=\mathrm{P}_{2} \mathrm{O}_{5} \quad$ Other $\%=62.7$

Other Formula $=\mathrm{La}_{2} \mathrm{O}_{3} \quad$ Other $\%=1.4$

Young's Modulus $=6.21 E 4$

1 st Technique $=$ Ind. Crk. Len.

$K_{\mathrm{IC}}=.41$

$\% \mathrm{CaO}=$

$\% \mathrm{PbO}=$

Gamma $=$

2nd Technique $=$

$K_{\mathrm{IC}}=$

Gamma $=1.35$

$n=$

Environment $=$ Heptane

Reference $=$ FREIMAN,S.W., Private Communications, 1984

Comments $=$ Compo.- 3 rd Other Oxide- $4.1 \% \quad \mathrm{Nd}_{2} \mathrm{O}_{3}$ 
Manufacturer $=$

$\begin{array}{ll}\% \mathrm{Al}_{2} \mathrm{O}_{3}=9.4 & \% \mathrm{~B}_{2} \mathrm{O}_{3}= \\ \% \mathrm{Na}_{2} \mathrm{O}= & \% \mathrm{~K}_{2} \mathrm{O}=28.3\end{array}$

Other Formula $=\mathrm{P}_{2} \mathrm{O}_{5} \quad$ Other $\%=56.7$

Other Formula $=\mathrm{La}_{2} \mathrm{O}_{3} \quad$ Other $\%=1.5$

Young's Modulus $=4.94 E 4$

lst Technique $=$ Ind. Crk. Len.

2nd Technique $=$

$n=$

Environment $=$ Heptane

Reference $=$ FREIMAN,S.W., Private Communications, 1984

Comments = Compo. -3 rd Other Oxide- $4.0 \% \quad \mathrm{Nd}_{2} \mathrm{O}_{3}$

Specific Material $=$ UP84-6957

Manufacturer $=$

$\% \mathrm{Al}_{2} \mathrm{O}_{3}=9.4$

$\% \mathrm{Na}_{2} \mathrm{O}=$

$\% \mathrm{~B}_{2} \mathrm{O}_{3}=$

$\% \mathrm{~K}_{2} \mathrm{O}=18.8$

Other Formula $=\mathrm{P}_{2} \mathrm{O}_{5}$

Other $\%=65.9$

Other Formula $=\mathrm{La}_{2} \mathrm{O}_{3}$

Other $\%=1.7$

Young's Modulus $=5.38 E 4$

1st Technique $=$ Ind. Crk. Len.

2nd Technique $=$

$n=$

Environment $=$ Heptane

Reference $=$ FREIMAN,S.W., Private Communications, 1984

Comments $=$ Compo. -3 rd Other Oxide- $4.2 \% \quad \mathrm{Nd}_{2} \mathrm{O}_{3}$

Specific Material = UP85-6959

Manufacturer $=$

$\% \mathrm{Al}_{2} \mathrm{O}_{3}=9.4$

$\% \mathrm{~B}_{2} \mathrm{O}_{3}=$

$\% \mathrm{Na}_{2} \mathrm{O}=$

$\% \mathrm{~K}_{2} \mathrm{O}=14.1$

Other Formula $=\mathrm{P}_{2} \mathrm{O}_{5}$

Other $\%=70.6$

Other Formula $=\mathrm{La}_{2} \mathrm{O}_{3} \quad$ Other \% $=1.6$

Young's Modulus $=5.68 E 4$

1st Technique $=$ Ind. Crk. Len.

2nd Technique $=$

$n=$

Environment $=$ Heptane

Reference $=$ FREIMAN,S.W., Private Communications, 1984

Comments $=$ Compo.- 3 rd Other Oxide- $4.2 \% \quad \mathrm{Nd}_{2} \mathrm{O}_{3}$

Specific Material = UP83-6955

Manufacturer $=$

$\% \mathrm{Al}_{2} \mathrm{O}_{3}=18.8$

$\% \mathrm{Na}_{2} \mathrm{O}=$

$\% \mathrm{~B}_{2} \mathrm{O}_{3}=$

$\% \mathrm{~K}_{2} \mathrm{O}=9.4$

Other Formula $=\mathrm{P}_{2} \mathrm{O}_{5} \quad$ Other \% $=65.9$

Other Formula $=\mathrm{La}_{2} \mathrm{O}_{3} \quad$ Other $\%=1.7$

Young's Modulus $=6.48 E 4$

1st Technique $=$ Ind. Crk. Len.

2nd Technique $=$

$n=$

Environment $=$ Heptane

Reference $=$ FREIMAN,S.W., Private Communications, 1984

Comments = Compo.- 3 rd Other Oxide- $4.2 \% \quad \mathrm{Nd}_{2} \mathrm{O}_{3}$
$\% \mathrm{CaO}=$

$\% \mathrm{PbO}=$

$K_{\mathrm{IC}}=.31$

$K_{\mathrm{IC}}=$

Gamma $=.97$

Gamma $=$
$K_{\mathrm{IC}}=.37$

$K_{\mathrm{IC}}=$

$\% \mathrm{CaO}=$

$\% \mathrm{PbO}=$

$\% \mathrm{MgO}=$

$\% \mathrm{SiO}_{2}=$

Gamma $=1.27$

Gamma =
$\% \mathrm{CaO}=$

$\% \mathrm{PbO}=$

$K_{\mathrm{IC}}=.38$

$K_{\mathrm{IC}}=$
$\% \mathrm{MgO}=$

$\% \mathrm{SiO}_{2}=$

Gamma $=1.27$

Gamma $=$

$\% \mathrm{CaO}=$

$\% \mathrm{PbO}=$

$K_{\mathrm{IC}}=.55$

$K_{\mathrm{IC}}=$
Gamma $=2.33$

Gamma =
\% $\mathrm{MgO}=$

$\% \mathrm{SiO}_{2}=$ 


\section{GUP83}

Specific Material $=$ E-Glass

Manufacturer $=$

$\% \mathrm{Al}_{2} \mathrm{O}_{3}=13.8$

$\% \quad \mathrm{Na}_{2} \mathrm{O}=5.44$

Other Formula =

Other Formula =

$\% \mathrm{~B}_{2} \mathrm{O}_{3}=8.07$

$\% \mathrm{~K}_{2} \mathrm{O}=$

Other $\%=$

Other $\%=$
$\% \mathrm{CaO}=17.4$

$\% \mathrm{PbO}=$

$\% \mathrm{MgO}=6.66$

$\% \mathrm{SiO}_{2}=53.8$

Young's Modulus =

1st Technique $=$ Dynamic Fat.

$K_{\mathrm{IC}}=$

Gamma $=$

2nd Technique $=$

$K_{\mathrm{IC}}=$

Gamma $=$

$n=24.8$

Environment $=$ air

Reference = GUPTA, P., Frac. Mech. of Ceram., Vol. 5, 291-303, 1983

Comments $=$ Material is an E-Glass Fiber

\section{KEN74}

Specific Material =

Manufacturer $=$

$\% \mathrm{Al}_{2} \mathrm{O}_{3}=$

$\% \mathrm{Na}_{2} \mathrm{O}=33$

Other Formula =

$\% \mathrm{~B}_{2} \mathrm{O}_{3}=$

$\% \mathrm{~K}_{2} \mathrm{O}=$

Other $\%=$

Other Formula =

Young's Modulus $=8.9 E 4$

1st Technique $=\mathrm{DCB}$

2nd Technique $=$

$n=$

Other $\%=$
$\% \mathrm{CaO}=$

$\% \mathrm{PbO}=$

$K_{\text {IC }}=1.12$

$K_{\mathrm{IC}}=$
$\% \mathrm{MgO}=$

$\% \mathrm{SiO}_{2}=67$

Environment $=\mathrm{N}_{2}(1)$

Reference = KENNEDY,C.,BRADT,R.,Frac. Mech. of Cer.,883-93,Vol. 2,1974

Comments $=$

Specific Material =

Manufacturer $=$

$\% \mathrm{Al}_{2} \mathrm{O}_{3}=$

$\% \mathrm{Na}_{2} \mathrm{O}=29$

Other Formula =

Other Formula =

Young's Modulus $=8.93 E 4$

1st Technique $=\mathrm{DCB}$

2nd Technique $=$

$\% \mathrm{MgO}=$

$\% \mathrm{~K}_{2} \mathrm{O}=$

Other $\%=$

$\% \mathrm{SiO}_{2}=71$

Other $\%=$

$\mathrm{Gamma}=7.0$

Gamma $=$

$n=$

Environment $=\mathrm{N}_{2}(1)$

Reference $=$ KENNEDY,C.,BRADT,R.,Frac. Mech. of Cer.,883-93,Vol. 2,1974

Comments $=$

Specific Material $=$

Manufacturer $=$

$\% \mathrm{Al}_{2} \mathrm{O}_{3}=$

$\% \mathrm{Na}_{2} \mathrm{O}=25$

Other Formula $=$

Other Formula =

Young's Modulus $=9.1 E 4$

1st Technique $=\mathrm{DCB}$

2nd Technique $=$

$n=$

Environment $=\mathrm{N}_{2}(1)$

Reference $=$ KENNEDY,C.,BRADT,R.,Frac. Mech. of Cer.,883-93,Vol. 2,1974

Comments $=$

$$
\begin{array}{ll}
\% \mathrm{~B}_{2} \mathrm{O}_{3}= \\
\% \mathrm{~K}_{2} \mathrm{O}= \\
& \text { Other } \%= \\
& \text { Other } \%=
\end{array}
$$

$$
K_{\mathrm{IC}}=1.04
$$$$
K_{\mathrm{IC}}=
$$

$\% \mathrm{MgO}=$

$\% \mathrm{SiO}_{2}=75$

$\mathrm{Gamma}=6.0$

$\mathrm{Gamma}=$

Gamma $=5.95$

Gamma $=$ 
Specific Material =

Manufacturer $=$

$\% \mathrm{Al}_{2} \mathrm{O}_{3}=$

$\% \mathrm{Na}_{2} \mathrm{O}=20$

Other Formula =

Other Formula =

Young's Modulus $=9.25 E 4$

1st Technique $=\mathrm{DCB}$

2nd Technique $=$
$\% \mathrm{~B}_{2} \mathrm{O}_{3}=$

$\% \mathrm{~K}_{2} \mathrm{O}=$

Other $\%=$

Other $\%=$
$\% \mathrm{CaO}=$

$\% \mathrm{PbO}=$

$K_{\mathrm{IC}}=.98$

$K_{\text {IC }}=$
$\% \mathrm{MgO}=$

$\% \mathrm{SiO}_{2}=80$

Gamma $=5.2$

Gamma $=$ $n=$

Environment $=\mathrm{N}_{2}(1)$

Reference = KENNEDY,C.,BRADT,R.,Frac. Mech. of Cer.,883-93,Vol. 2,1974

Comments $=$

Specific Material $=$

Manufacturer $=$
$\% \mathrm{Al}_{2} \mathrm{O}_{3}=$

$\% \mathrm{Na}_{2} \mathrm{O}=17$

Other Formula $=$

Other Formula =

Young's Modulus $=9.35 E 4$

1st Technique $=\mathrm{DCB}$

2nd Technique $=$
$\% \mathrm{CaO}=$

$\% \mathrm{PbO}=$

Other $\%=$

Other $\%=$
$\% \mathrm{MgO}=$

$\% \mathrm{SiO}_{2}=83$

$K_{\text {IC }}=.96$

$K_{\mathrm{IC}}=$

Gamma
Gamma $=4.95$

$n=$

Environment $=\mathrm{N}_{2}(\mathrm{l})$

Reference = KENNEDY,C.,BRADT,R.,Frac. Mech. of Cer.,883-93,Vol. 2,1974

Comments $=$

Specific Material $=$

Manufacturer =

$\% \mathrm{Al}_{2} \mathrm{O}_{3}=$

$\% \mathrm{Na}_{2} \mathrm{O}=$

Other Formula $=$

Other Formula $=$

Young's Modulus $=10.1 E 4$

1st Technique $=\mathrm{DCB}$

2nd Technique $=$

$n=$

Environment $=\mathrm{N}_{2}(\mathrm{l})$

Reference = KENNEDY,C.,BRADT,R.,Frac. Mech. of Cer.,883-93,Vol. 2,1974

Comments $=$

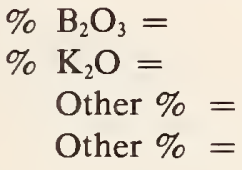

$\% \mathrm{CaO}=$

$K_{\text {IC }}=.87$

$K_{\mathrm{IC}}=$

\section{MEC74}

Specific Material $=7940$

Manufacturer $=$ Corning Glass

$\% \mathrm{Al}_{2} \mathrm{O}_{3}=$

$\% \mathrm{Na}_{2} \mathrm{O}=$

Other Formula =

Other Formula =

Young's Modulus = 7.21 E4

1st Technique $=\mathrm{AMDCB}$

2nd Technique $=$

$n=$

Environment $=$ air

Reference = MECHOLSKY,J.,RICE,R., JACTAW, Vol. 57, No. 10, 1974

Comments $=$

$\% \mathrm{~B}_{2} \mathrm{O}_{3}=$

$\% \mathrm{~K}_{2} \mathrm{O}=$

Other $\%=$

Other $\%=$
$\% \mathrm{MgO}=$

$\% \mathrm{SiO}_{2}=100$

$\% \mathrm{CaO}=$

$\% \mathrm{MgO}=$

Gamma $=3.7$

Gamma $=$
$\% \mathrm{SiO}_{2}=100$

$K_{\text {IC }}=.73$

$K_{\mathrm{IC}}=$

Gamma $=3.7$

Gamma $=$ 
Specific Material $=1723$

Manufacturer $=$ Corning Glass

$\% \mathrm{Al}_{2} \mathrm{O}_{3}=9.43 \quad \% \mathrm{~B}_{2} \mathrm{O}_{3}=4.60$

$\% \mathrm{~K}_{2} \mathrm{O}=$

$\% \mathrm{CaO}=11.4$

$\% \mathrm{PbO}=$

$\% \mathrm{MgO}=11.1$

$\% \mathrm{Na}_{2} \mathrm{O}=$

Other $\%=2.51$

Other Formula $=\mathrm{BaO}$

Other $\%=$

$\% \mathrm{SiO}_{2}=60.8$

Other Formula =

Young's Modulus $=8.91 E 4$

1 st Technique $=\mathrm{AMDCB}$

$K_{\mathrm{IC}}=.81$

2nd Technique $=$

$K_{\mathrm{IC}}=$

Gamma $=3.7$

$n=$

Environment $=$ air

Reference = MECHOLSKY,J.,RICE,R., JACTAW, Vol. 57, No. 10, 1974

Comments $=$

Specific Material $=7740$

Manufacturer $=$ Corning Glass

$\begin{array}{ll}\% \mathrm{Al}_{2} \mathrm{O}_{3}=3.36 & \% \mathrm{~B}_{2} \mathrm{O}_{3}=11.3 \\ \% \mathrm{Na}_{2} \mathrm{O}=3.26 & \% \mathrm{~K}_{2} \mathrm{O}= \\ \text { Other Formula }= & \text { Other } \%= \\ \text { Other Formula }= & \text { Other } \%=\end{array}$

$\% \mathrm{CaO}=$

$\% \mathrm{PbO}=$

Gamma $=$

Young's Modulus $=6.37 E 4$

1 st Technique $=\mathrm{AMDCB}$

2nd Technique $=$

$n=$

Environment $=$ air

Reference = MECHOLSKY,J.,RICE,R., JACTAW, Vol. 57, No. 10, 1974

Comments $=$

Specific Material $=$

Manufacturer $=$

$$
\begin{aligned}
& K_{\mathrm{IC}}=.71 \\
& K_{\mathrm{IC}}=
\end{aligned}
$$

Gamma $=4.0$

Gamma $=$

$\% \mathrm{Al}_{2} \mathrm{O}_{3}=.581$
$\% \mathrm{~B}_{2} \mathrm{O}_{3}=$

$\% \mathrm{~K}_{2} \mathrm{O}=$

Other $\%=$

Other $\%=$
Other Formula =

Other Formula =

Young's Modulus $=7.34 E 4$

1st Technique $=\mathrm{AMDCB}$

2nd Technique $=$

$n=$

Environment $=$ air

Reference = MECHOLSKY,J.,RICE,R., JACTAW, Vol. 57, No. 10, 1974

Comments $=$

\section{MEC77}

Specific Material $=$

Manufacturer =
$\% \mathrm{Al}_{2} \mathrm{O}_{3}=$
$\% \mathrm{~B}_{2} \mathrm{O}_{3}=$
$\% \mathrm{Na}_{2} \mathrm{O}=$
$\% \mathrm{~K}_{2} \mathrm{O}=$
Other Formula $=\mathrm{Li}_{2} \mathrm{O}$
Other $\%=33$
Other Formula =
Other $\%=$

$K_{\mathrm{IC}}=.72$

$K_{\mathrm{IC}}=$
$\% \mathrm{CaO}=5.28$

$\% \mathrm{PbO}=$

Young's Modulus $=5.2 \mathrm{E} 4$

1 st Technique $=\mathrm{DCB}$

2nd Technique $=$

$n=$

Environment $=$ air

Reference $=$ MECHOLSKY,J.,FREIMAN,Proc. of Int. Glass Cong.,479-83, 1977

Comments $=$

$$
\begin{array}{ll}
K_{\mathrm{IC}}=.7 & \text { Gamma }=5.0 \\
K_{\mathrm{IC}}= & \text { Gamma }=
\end{array}
$$

Gamma $=$ 
Speciric Material =

Manufacturer =

$\% \mathrm{Al}_{2} \mathrm{O}_{3}=$

$\% \mathrm{~B}_{2} \mathrm{O}_{3}=$

$\% \mathrm{~K}_{2} \mathrm{O}=$

$\% \mathrm{CaO}=$

$\% \mathrm{MgO}=$

$\% \mathrm{Na}_{2} \mathrm{O}=$

Other $\%=37.5$

$\% \mathrm{PbO}=$

$\% \mathrm{SiO}_{2}=62.5$

Other Formula $=\mathrm{BaO}$

Other $\%=$

Young's Modulus $=6.4 E 4$

1st Technique $=\mathrm{DCB}$

2nd Technique $=$

$K_{\text {IC }}=.5$

$K_{\text {IC }}=$

Gamma $=2.0$

$n=$

Environment $=$ air

Reference $=$ MECHOLSKY,J.,FREIMAN,Proc. of Int. Glass Cong.,479-83, 1977

Comments $=$

\section{MET72}

Specific Material $=$ ENK5

Manufacturer $=$ Owens-Corning
$\% \mathrm{Al}_{2} \mathrm{O}_{3}=8.55$
$\% \mathrm{~B}_{2} \mathrm{O}_{3}=8.17$
$\% \mathrm{Na}_{2} \mathrm{O}=2.14$
$\% \mathrm{~K}_{2} \mathrm{O}=2.21$
Other $\%=$
Other Formula =
Other $\%=$
Other Formula =

$\% \mathrm{CaO}=17.6$

$\% \mathrm{PbO}=$

Gamma $=$

$\% \mathrm{MgO}=6.74$

$\% \mathrm{SiO}_{2}=54.4$

Young's Modulus =

1st Technique $=$ Dynamic Fat.

2nd Technique $=$

$K_{\text {IC }}=$

Gamma $=$

$n=11$

Environment $=\mathrm{H}_{2} \mathrm{O}$

Reference = METCALFE,A.G.,SCHMITZ,G.K.,Glass Tech.,5-16,Vol. 13,No. 1,1972

Comments $=$ Material is an E-Glass Fiber

Specific Material $=$ EF5

Manufacturer $=$ Owens-Corning
$\% \mathrm{Al}_{2} \mathrm{O}_{3}=8.94$
$\% \mathrm{~B}_{2} \mathrm{O}_{3}=8.54$
$\% \mathrm{Na}_{2} \mathrm{O}=$
$\% \mathrm{~K}_{2} \mathrm{O}=$
Other $\%=$
Other Formula $=$
Other Formula $=$
Other $\%=$

$\% \mathrm{CaO}=18.4$

$\% \mathrm{PbO}=$

Young's Modulus =

1st Technique $=$ Dynamic Fat.

2nd Technique $=$

$n=19$

$K_{\text {IC }}=$

Gamma $=$

Environment $=\mathrm{H}_{2} \mathrm{O}$

Reference = METCALFE,A.G.,SCHMITZ,G.K.,Glass Tech.,5-16,Vol. 13,No. 1,1972

Comments $=$ Material is an E-Glass Fiber

Specific Material $=$ EBN0

Manufacturer $=$ Owens-Corning

$\% \mathrm{Al}_{2} \mathrm{O}_{3}=9.83 \quad \% \mathrm{~B}_{2} \mathrm{O}_{3}=$

$\% \mathrm{Na}_{2} \mathrm{O}=$

$\% \mathrm{~K}_{2} \mathrm{O}=$

$\% \mathrm{CaO}=21.3$

$\% \mathrm{PbO}=$

Gamma $=$

$K_{\mathrm{IC}}=$

Gamma $=$

Other Formula =

Other $\%=$

Other Formula =

Other $\%=$

Young's Modulus =

1st Technique $=$ Dynamic Fat.

2nd Technique $=$

$K_{\mathrm{IC}}=$

$\% \mathrm{MgO}=7.04$

$\% \mathrm{SiO}_{2}=56.9$

$n=30$

Environment $=\mathrm{H}_{2} \mathrm{O}$

Reference = METCALFE,A.G.,SCHMITZ,G.K.,Glass Tech.,5-16,Vol.13,No. 1,1972

Comments = Material is an E-Glass Fiber

$$
\begin{array}{ll}
K_{\mathrm{IC}}= & \text { Gamma }= \\
K_{\text {IC }}= & \text { Gamma }=
\end{array}
$$


Specific Material $=$ ES

Manufacturer $=$ Owens-Corning

$\% \mathrm{Al}_{2} \mathrm{O}_{3}=8.89 \quad \% \mathrm{~B}_{2} \mathrm{O}_{3}=8.53$

$\% \mathrm{Na}_{2} \mathrm{O}=.504$

Other Formula =

$\% \mathrm{~K}_{2} \mathrm{O}=$

$\% \mathrm{CaO}=18.3$

$\% \mathrm{MgO}=6.97$

Other $\%=$

Other Formula =

Other $\%=$

$\% \mathrm{PbO}=$

$\% \mathrm{SiO}_{2}=56.7$

Young's Modulus =

1st Technique $=$ Dynamic Fat.

2nd Technique $=$

$n=22$

Environment $=\mathrm{H}_{2} \mathrm{O}$

Reference = METCALFE,A.G.,SCHMITZ,G.K.,Glass Tech.,5-16,Vol. 13,No. 1,1972

Comments $=$ Material is an E-Glass Fiber

Specific Material $=$ ENO

Manufacturer $=$ Owens-Corning

$\% \mathrm{Al}_{2} \mathrm{O}_{3}=8.88 \quad \% \mathrm{~B}_{2} \mathrm{O}_{3}=8.52$

$\% \mathrm{Na}_{2} \mathrm{O}=$

$\% \mathrm{~K}_{2} \mathrm{O}=$

$\% \mathrm{CaO}=18.3$

$K_{\mathrm{IC}}=$

Gamma =

$K_{\mathrm{IC}}=$

Gamma $=$

Other Formula =

Other Formula =

Other $\%=$

$\% \mathrm{PbO}=$

$\% \mathrm{MgO}=6.97$

Other $\%=$

$\% \mathrm{SiO}_{2}=57.2$

Young's Modulus =

1st Technique $=$ Dynamic Fat.

2nd Technique $=$

$n=22$

Environment $=\mathrm{H}_{2} \mathrm{O}$

Reference = METCALFE,A.G.,SCHMITZ,G.K.,Glass Tech.,5-16,Vol. 13,No. 1,1972

Comments = Material is an E-Glass Fiber

Specific Material $=$ EN5

Manufacturer $=$ Owens-Corning

$\% \mathrm{Al}_{2} \mathrm{O}_{3}=8.45 \quad \% \mathrm{~B}_{2} \mathrm{O}_{3}=8.07$

$\% \mathrm{Na}_{2} \mathrm{O}=5.44$

$\% \mathrm{~K}_{2} \mathrm{O}=$

$\% \mathrm{CaO}=17.4$

$K_{\mathrm{IC}}=$

Gamma $=$

$K_{\mathrm{IC}}=$

Gamma $=$

Other Formula =

Other $\%=$

Other Formula =

Other $\%=$

$\% \mathrm{PbO}=$

$\% \mathrm{MgO}=6.66$

$\% \mathrm{SiO}_{2}=53.8$

Young's Modulus =

lst Technique $=$ Dynamic Fat.

2nd Technique $=$

$n=20$

Environment $=\mathrm{H}_{2} \mathrm{O}$

Reference = METCALFE,A.G.,SCHMITZ,G.K.,Glass Tech.,5-16,Vol. 13,No. 1,1972

Comments $=$ Material is an E-Glass Fiber

Specific Material $=$ EK5

Manufacturer $=$ Owens-Corning

$\%, \mathrm{Al}_{2} \mathrm{O}_{3}=8.61 \quad \% \mathrm{~B}_{2} \mathrm{O}_{3}=8.23$

$\% \mathrm{Na}_{2} \mathrm{O}=\quad \% \mathrm{~K}_{2} \mathrm{O}=3.64$

Other Formula $=\quad$ Other $\%=$

$K_{\mathrm{IC}}=\quad$ Gamma $=$

$K_{\mathrm{IC}}=\quad$ Gamma $=$

Other Formula $=$

Other $\%=$

Young's Modulus =

1st Technique $=$ Dynamic Fat.

2nd Technique $=$

$n=17$

Environment $=\mathrm{H}_{2} \mathrm{O}$

Reference = METCALFE,A.G.,SCHMITZ,G.K.,Glass Tech.,5-16,Vol. 13,No. 1,1972

Comments $=$ Material is an E-Glass Fiber

$\begin{array}{ll}K_{\mathrm{IC}}= & \text { Gamma }= \\ K_{\mathrm{IC}}= & \text { Gamma }=\end{array}$


Specific Material $=$

Manufacturer $=$

$\% \mathrm{Al}_{2} \mathrm{O}_{3}=$

$\% \mathrm{~B}_{2} \mathrm{O}_{3}=100$

$\% \mathrm{~K}_{2} \mathrm{O}=$

Other \% =

Other Formula =

Other Formula =

Young's Modulus =

1st Technique $=$ Ind. Crk. Len.

2nd Technique $=$

$n=$

Environment $=$ air

Reference = MIYATA,N.,JINNO,H.J. of Non-Cryst. Sol. 38 \& 39,391-96,1980

Comments $=$

Specific Material =

Manufacturer $=$

$\% \mathrm{Al}_{2} \mathrm{O}_{3}=$

$\% \mathrm{~B}_{2} \mathrm{O}_{3}=98.4$

$\% \mathrm{~K}_{2} \mathrm{O}=$

$\% \mathrm{CaO}=$

$\% \mathrm{PbO}=1.5$

Other $\%=$

Other Formula =

Other Formula =

Young's Modulus =

1st Technique $=$ Ind. Crk. Len

2nd Technique $=$

$n=$

Environment $=$ air

Reference = MIYATA,N.,JINNO,H.,J. of Non-Cryst. Sol. 38 \& 39,391-96,1980

Comments $=$

Specific Material $=$

Manufacturer =

$\% \mathrm{Al}_{2} \mathrm{O}_{3}=$

$\% \mathrm{Na}_{2} \mathrm{O}=$

Other Formula =

Other Formula =

Young's Modulus =

1st Technique $=$ Ind. Crk. Len.

$\% \mathrm{~B}_{2} \mathrm{O}_{3}=96.8$

$\% \mathrm{~K}_{2} \mathrm{O}=$

Other $\%=$

$K_{\mathrm{IC}}=.87$

$K_{\mathrm{IC}}=$

$K_{\mathrm{IC}}=.89$

$K_{\text {IC }}=$

$\% \mathrm{CaO}=$

$\% \mathrm{PbO}=$

$\% \mathrm{MgO}=$

$\% \mathrm{SiO}_{2}=$

Other $\%=$

Gamma =

Gamma =

Other $\%=$

Gamma $=$

Gamma $=$

$\% \mathrm{MgO}=$

$\% \mathrm{SiO}_{2}=$
$\% \mathrm{CaO}=$

$\% \mathrm{PbO}=3.13$

$\% \mathrm{MgO}=$

$\% \mathrm{SiO}_{2}=$

$$
K_{\mathrm{IC}}=.8
$$$$
K_{\mathrm{IC}}=
$$

Gamma $=$

Gamma $=$

2nd Technique $=$

$n=$

Environment $=$ air

Reference = MIYATA,N.,JINNO,H.,J. of Non-Cryst. Sol. 38 \& 39,391-96,1980

Comments $=$

Specific Material =

Manufacturer =

$\% \mathrm{Al}_{2} \mathrm{O}_{3}=$

$\% \mathrm{Na}_{2} \mathrm{O}=$

Other Formula =

Other Formula =

Young's Modulus =

lst Technique $=$ Ind. Crk. Len.

2nd Technique $=\mathrm{NB}$

$\% \mathrm{~B}_{2} \mathrm{O}_{3}=95.8$

$\% \mathrm{~K}_{2} \mathrm{O}=$

Other \% =

Other $\%=$

$n=$

Environment $=$ air

Reference = MIYATA,N.,JINNO,H.,J. of Non-Cryst. Sol. 38 \& 39,391-96,1980

Comments $=$

\footnotetext{
$K_{\text {IC }}=.77$

$K_{\mathrm{IC}}=.82$
}

$\% \mathrm{CaO}=$

$\% \mathrm{PbO}=4.16$

$\% \mathrm{MgO}=$

$\% \mathrm{SiO}_{2}=$

Gamma $=$

Gamma $=$ 
$\% \mathrm{~B}_{2} \mathrm{O}_{3}=94.3$

$\% \mathrm{Na}_{2} \mathrm{O}=$

Other Formula =

Other Formula =

Young's Modulus =

1st Technique $=$ Ind. Crk. Len.

2nd Technique $=\mathrm{NB}$

$\% \mathrm{~K}_{2} \mathrm{O}=$

Other \% =

Other $\%=$
$\% \mathrm{CaO}=$

$\% \mathrm{PbO}=5.62$

$\% \mathrm{MgO}=$

$\% \mathrm{SiO}_{2}=$

$K_{\mathrm{IC}}=.78$

$K_{\mathrm{IC}}=.84$
Gamma $=$

Gamma $=$

$n=$

Environment $=$ air

Reference = MIYATA,N.,JINNO,H.,J. of Non-Cryst. Sol. 38 \& 39,391-96,1980

Comments $=$

Specific Material $=$

Manufacturer $=$

$\% \mathrm{Al}_{2} \mathrm{O}_{3}=$

$\% \mathrm{Na}_{2} \mathrm{O}=$

Other Formula =

Other Formula =

Young's Modulus =

1st Technique $=$ Ind. Crk. Len.

2nd Technique $=\mathrm{NB}$

$\% \mathrm{~B}_{2} \mathrm{O}_{3}=87.9$

$\% \mathrm{~K}_{2} \mathrm{O}=$

Other $\%=$

Other $\%=$
$\% \mathrm{CaO}=$

$\% \mathrm{PbO}=12.0$

$\% \mathrm{MgO}=$

$\% \mathrm{SiO}_{2}=$

$K_{\mathrm{IC}}=1.63$

$K_{\mathrm{IC}}=1.59$

$n=$

Environment $=$ air

Reference = MIYATA,N.,JINNO,H.,J. of Non-Cryst. Sol. 38 \& 39,391-96,1980

Comments $=$

Specific Material $=$

Manufacturer $=$

$\% \mathrm{Al}_{2} \mathrm{O}_{3}=$

Other Formula =

Other Formula =
$\% \mathrm{CaO}=$

$\% \mathrm{PbO}=14.0$

Other \% =
Gamma $=$ Gamma

Young's Modulus =

1st Technique $=$ Ind. Crk. Len.

2nd Technique $=\mathrm{NB}$

$n=$

Environment $=$ air

Reference = MIYATA,N.,JINNO,H.,J. of Non-Cryst. Sol. 38 \& 39,391-96,1980

Comments $=$

Specific Material $=$

Manufacturer =

$\% \mathrm{Al}_{2} \mathrm{O}_{3}=$

$\% \mathrm{Na}_{2} \mathrm{O}=$

Other Formula $=$

Other Formula =

Young's Modulus =

1st Technique $=$ Ind. Crk. Len.

2nd Technique $=$ NB

$n=$

Environment $=$ air

Reference = MIYATA,N.,JINNO,H.,J. of Non-Cryst. Sol. 38 \& 39,391-96,1980

Comments $=$

$$
\begin{array}{ll}
\% \mathrm{~B}_{2} \mathrm{O}_{3}=83.7 \\
\% \mathrm{~K}_{2} \mathrm{O}= \\
& \text { Other \% }= \\
& \text { Other \% }=
\end{array}
$$

$\% \mathrm{~B}_{2} \mathrm{O}_{3}=85.9$

$\% \mathrm{~K}_{2} \mathrm{O}=$

Other $\%=$

$K_{\text {IC }}=1.39$

$K_{\mathrm{IC}}=1.38$

Gamma $=$

Gamma =
$\% \mathrm{CaO}=$

$\% \mathrm{PbO}=16.2$

$K_{\mathrm{IC}}=1.29$

$K_{\text {IC }}=1.33$
$\% \mathrm{MgO}=$

$\% \mathrm{SiO}_{2}=$ 
Specific Material $=$

Manufacturer =
$\% \mathrm{Al}_{2} \mathrm{O}_{3}=$
$\% \mathrm{~B}_{2} \mathrm{O}_{3}=80.7$
$\% \mathrm{Na}_{2} \mathrm{O}=$
$\% \mathrm{~K}_{2} \mathrm{O}=$
Other Formula =
Other \% =
Other Formula =
Other $\%=$

$\% \mathrm{CaO}=$

$\% \mathrm{MgO}=$

$\% \mathrm{PbO}=19.2 \quad \% \mathrm{SiO}_{2}=$

Young's Modulus =

1st Technique $=$ Ind. Crk. Len.

$K_{\mathrm{IC}}=.95$

$K_{\mathrm{IC}}=.91$

Gamma $=$

2nd Technique $=\mathrm{NB}$

Gamma $=$

$n=$

Environment $=$ air

Reference = MIYATA,N.,JINNO,H.,J. of Non-Cryst. Sol. 38 \& 39,391-96, 1980

Comments $=$

MIY81

Specific Material $=$

Manufacturer $=$

$\% \mathrm{Al}_{2} \mathrm{O}_{3}=$

$\% \mathrm{~B}_{2} \mathrm{O}_{3}=100$

$\% \mathrm{CaO}=$

$\% \mathrm{MgO}=$

$\% \mathrm{Na}_{2} \mathrm{O}=$

$\% \mathrm{~K}_{2} \mathrm{O}=$

$\% \mathrm{PbO}=$

$\% \mathrm{SiO}_{2}=$

Other Formula =

Other Formula $=$

Other $\%=$

Other $\%=$

Young's Modulus $=1.73 E 4$

1 st Technique $=$ Ind. Crk. Len.

$K_{\mathrm{IC}}=.96$

Gamma $=26.64$

2nd Technique $=$

$K_{\mathrm{IC}}=$

Gamma $=$

$n=$

Environment $=$ air

Reference = MIYATA,H.,JINNO,N.,Journ. of Mat. Sci. 16,2205-17,1981

Comments $=$

Specific Material =

Manufacturer =

$\% \mathrm{Al}_{2} \mathrm{O}_{3}=$

$\% \mathrm{~B}_{2} \mathrm{O}_{3}=98.4$

$\% \mathrm{CaO}=$

$\% \mathrm{PbO}=1.50$

$\% \mathrm{MgO}=$

$\% \mathrm{Na}_{2} \mathrm{O}=$

$\% \mathrm{~K}_{2} \mathrm{O}=$

$\% \mathrm{SiO}_{2}=$

Other Formula $=$

Other $\%=$

Other Formula =

Other $\%=$

Young's Modulus $=1.87 E 4$

1st Technique $=$ Ind. Crk. Len.

$K_{\mathrm{IC}}=.96$

$K_{\mathrm{lC}}=$

Gamma $=24.64$

2nd Technique $=$

$n=$

Environment $=$ air

Reference = MIYATA,H.,JINNO,N.,Journ. of Mat. Sci. 16,2205-17,1981

Comments $=$

Specific Material =

Manufacturer =

$\% \mathrm{Al}_{2} \mathrm{O}_{3}=$

$\% \mathrm{~B}_{2} \mathrm{O}_{3}=96.8$

$\% \mathrm{CaO}=$

$\% \mathrm{PbO}=3.13$

$\% \mathrm{MgO}=$

$\% \mathrm{Na}_{2} \mathrm{O}=$

$\% \mathrm{~K}_{2} \mathrm{O}=$

Other $\%=$

Gamma $=$

Other Formula =

Other Formula =

Other $\%=$

$\% \mathrm{SiO}_{2}=$

Young's Modulus $=2.02 E 4$

1st Technique $=$ Ind. Crk. Len.

$K_{\mathrm{IC}}=.88$

Gamma $=19.17$

2nd Technique $=$

$K_{\mathrm{IC}}=$

Gamma $=$

$n=$

Environment $=$ air

Reference = MIYATA,H.,JINNO,N.,Journ. of Mat. Sci. 16,2205-17,1981

Comments $=$ 
Specific Material =

Manufacturer $=$

$\% \mathrm{Al}_{2} \mathrm{O}_{3}=$

$\% \mathrm{~B}_{2} \mathrm{O}_{3}=95.8$

$\% \mathrm{CaO}=$

$\% \mathrm{PbO}=4.16$

$\% \mathrm{MgO}=$

$\% \mathrm{Na}_{2} \mathrm{O}=$

$\% \mathrm{~K}_{2} \mathrm{O}=$

Other $\%=$

Other Formula =

Other $\%=$

$\% \mathrm{SiO}_{2}=$

Other Formula =

Young's Modulus $=2.1 E 4$

1st Technique $=$ Ind. Crk. Len.

$K_{\mathrm{IC}}=.87$

$K_{\mathrm{lC}}=.82$

Gamma $=18.02$

2nd Technique $=\mathrm{NB}$

Gamma $=16.01$

$n=$

Environment $=$ air

Reference = MIYATA,H.,JINNO,N.,Journ. of Mat. Sci. 16,2205-17,1981

Comments $=$

Specific Material =

Manufacturer $=$

$\% \mathrm{Al}_{2} \mathrm{O}_{3}=$
$\% \mathrm{Na}_{2} \mathrm{O}=$

$\% \mathrm{~B}_{2} \mathrm{O}_{3}=94.3$

$\% \mathrm{CaO}=$

$\% \mathrm{PbO}=5.62$

$\% \mathrm{MgO}=$

Other Formula =

$\% \mathrm{~K}_{2} \mathrm{O}=$

Other $\%=$

Other Formula =

Other $\%=$

$\% \mathrm{SiO}_{2}=$

Young's Modulus $=2.23 E 4$

1st Technique $=$ Ind. Crk. Len.

$K_{\mathrm{IC}}=.88$

$K_{\mathrm{lC}}=.85$

Gamma $=17.36$

2nd Technique $=\mathrm{NB}$

Gamma $=16.20$

$n=$

Environment $=$ air

Reference = MIYATA,H.,JINNO,N.,Journ. of Mat. Sci. 16,2205-17,1981

Comments $=$

Specific Material =

Manufacturer =

$\% \mathrm{Al}_{2} \mathrm{O}_{3}=$

$\% \mathrm{~B}_{2} \mathrm{O}_{3}=87.9$

$\% \mathrm{~K}_{2} \mathrm{O}=$

$\% \mathrm{CaO}=$

$\% \mathrm{PbO}=12$

$\% \mathrm{MgO}=$

\% $\mathrm{Na}_{2} \mathrm{O}=$

Other $\%=$

Other Formula =

Other $\%=$

$\% \mathrm{SiO}_{2}=$

Other Formula =

Young's Modulus $=3.12 E 4$

1st Technique $=$ Ind. Crk. Len.

$K_{1 \mathrm{C}}=1.75$

Gamma $=49.08$

2nd Technique $=\mathrm{NB}$

$K_{\text {IC }}=1.6$

Gamma $=41.03$

$n=$

Environment $=$ air

Reference = MIYATA,H.,JINNO,N.,Journ. of Mat. Sci. 16,2205-17,1981

Comments $=$

Specific Material $=$

Manufacturer $=$

$\% \cdot \mathrm{Al}_{2} \mathrm{O}_{3}=$

$\% \mathrm{~B}_{2} \mathrm{O}_{3}=85.9$

$\% \mathrm{~K}_{2} \mathrm{O}=$

$\% \mathrm{CaO}=$

$\% \mathrm{PbO}=14.0$

$\% \mathrm{MgO}=$

$\% \mathrm{Na}_{2} \mathrm{O}=$

Other $\%=$

Other Formula =

Other $\%=$

$\% \mathrm{SiO}_{2}=$

Young's Modulus $=3.62 E 4$

$1 \mathrm{st}$ Technique $=$ Ind. Crk. Len.

$K_{\text {IC }}=1.52$

$K_{\text {IC }}=1.37$

Gamma $=31.91$

2nd Technique $=\mathrm{NB}$

Gamma $=25.92$

$n=$

Environment $=$ air

Reference = MIYATA,H.,JINNO,N.,Journ. of Mat. Sci. 16,2205-17,1981

Comments $=$ 
Specific Material =

Manufacturer =
$\% \mathrm{Al}_{2} \mathrm{O}_{3}=$
$\% \mathrm{~B}_{2} \mathrm{O}_{3}=83.7$
$\% \mathrm{Na}_{2} \mathrm{O}=$
$\% \mathrm{~K}_{2} \mathrm{O}=$
Other Formula =
Other $\%=$
Other Formula =
Other $\%=$

$\% \mathrm{CaO}=$

$\% \mathrm{MgO}=$

$\% \mathrm{PbO}=16.2$

$\% \mathrm{SiO}_{2}=$

Young's Modulus $=4.45 E 4$

1 st Technique $=$ Ind. Crk. Len.

$K_{\mathrm{IC}}=1.4$

$\mathrm{Gamma}=22.02$

2nd Technique $=\mathrm{NB}$

$K_{\text {IC }}=1.35$

Gamma $=20.48$

$n=$

Environment $=$ air

Reference = MIYATA,H.,JINNO,N.,Journ. of Mat. Sci. 16,2205-17,1981

Comments $=$

Specific Material $=$

Manufacturer $=$

$\begin{array}{ll}\% \mathrm{Al}_{2} \mathrm{O}_{3}= & \% \mathrm{~B}_{2} \mathrm{O}_{3}=80.7 \\ \% \mathrm{Na}_{2} \mathrm{O}= & \% \mathrm{~K}_{2} \mathrm{O}= \\ \text { Other Formula }= & \text { Other \%= } \\ \text { Other Formula }= & \text { Other \% }=\end{array}$

$\% \mathrm{CaO}=$

$\% \mathrm{PbO}=19.2$

$\% \mathrm{MgO}=$
$\% \mathrm{SiO}_{2}=$

Young's Modulus $=6.04 E 4$

1st Technique $=$ Ind. Crk. Len.

$K_{\mathrm{IC}}=1.05$

Gamma $=9.13$

2nd Technique $=\mathrm{NB}$

$K_{\mathrm{IC}}=.93$

Gamma $=7.16$

$n=$

Environment $=$ air

Reference = MIYATA,H.,JINNO,N.,Journ. of Mat. Sci. 16,2205-17,1981

Comments $=$

MIY83

Specific Material $=$

Manufacturer =

$\% \mathrm{Al}_{2} \mathrm{O}_{3}=$

$\% \mathrm{Na}_{2} \mathrm{O}=14.5$

Other Formula $=$

Other Formula =
$\% \mathrm{~B}_{2} \mathrm{O}_{3}=$

$\% \mathrm{~K}_{2} \mathrm{O}=$

Other $\%=$

Other $\%=$
$\% \mathrm{CaO}=5.36 \quad \% \mathrm{MgO}=$

$\% \mathrm{PbO}=\quad \% \mathrm{SiO}_{2}=80.0$

Young's Modulus $=6.19 E 4$

1st Technique $=\mathrm{NB}$

2nd Technique $=$

$n=$

Environment $=$ air

Reference = MIYATA,N.,TANIGAWA,K.,Frac. Mech. of Cer.,Vol. 5,609-23,1983

Comments $=$ Test Tech. - introduced a sharp crack at the notch

Specific Material =

Manufacturer $=$

$\% \mathrm{Al}_{2} \mathrm{O}_{3}=$

$\% \mathrm{Na}_{2} \mathrm{O}=19.4$

Other Formula =

Other Formula =

Young's Modulus $=6.28 E 4$

1st Technique $=\mathrm{NB}$

2nd Technique $=$

$K_{\mathrm{IC}}=.8$

$K_{\mathrm{IC}}=$

Gamma $=5.17$

Gamma =
$K_{\mathrm{IC}}=.8$

$K_{\mathrm{IC}}=$

$\% \mathrm{CaO}=5.37$

$\% \mathrm{PbO}=$

$\% \mathrm{MgO}=$

$\% \mathrm{SiO}_{2}=75.1$

Other $\%=$

Other $\%=$

$n=$

Environment $=$ air

Reference = MIYATA,N.,TANIGAWA,K.,Frac. Mech. of Cer.,Vol. 5,609-23,1983

Comments $=$ Test Tech. - introduced a sharp crack at the notch 
Specific Material $=$

Manufacturer =

$\% \mathrm{Al}_{2} \mathrm{O}_{3}=$

$\% \mathrm{Na}_{2} \mathrm{O}=24.3$

Other Formula =

$\% \mathrm{~B}_{2} \mathrm{O}_{3}=$

$\% \mathrm{~K}_{2} \mathrm{O}=$

Other $\%=$

Other $\%=$
$\% \quad \mathrm{CaO}=5.37$

$\% \mathrm{PbO}=$
$\% \mathrm{MgO}=$

$\% \mathrm{SiO}_{2}=70.2$

$K_{\mathrm{IC}}=.8$

$K_{\text {IC }}=$

Gamma $=5.02$

Gamma =

$n=$

Environment $=$ air

Reference = MIYATA,N.,TANIGAWA,K.,Frac. Mech. of Cer., Vol. 5,609-23,1983

Comments $=$ Test Tech. - introduced a sharp crack at the notch

Specific Material =

Manufacturer $=$

$\% \mathrm{Al}_{2} \mathrm{O}_{3}=$

$\% \mathrm{Na}_{2} \mathrm{O}=29.2$

Other Formula =

Other Formula =

$\% \mathrm{~B}_{2} \mathrm{O}_{3}=$

$\% \mathrm{~K}_{2} \mathrm{O}=$ Other \% =

Other $\%=$
$\% \mathrm{CaO}=5.38$

$\% \mathrm{PbO}=$

$\% \mathrm{MgO}=$

$\% \mathrm{SiO}_{2}=65.3$

$K_{\mathrm{IC}}=.8$

$K_{\mathrm{IC}}=$

Gamma $=4.98$

Gamma $=$

$n=$

Environment $=$ air

Reference = MIYATA,N.,TANIGAWA,K.,Frac. Mech. of Cer.,Vol. 5,609-23,1983

Comments $=$ Test Tech.- introduced a sharp crack at the notch

\section{MOU59}

Specific Material =

Manufacturer =

$\% \mathrm{Al}_{2} \mathrm{O}_{3}=1.16$

$\% \mathrm{Na}_{2} \mathrm{O}=13.4$

Other Formula =

Other Formula =

Young's Modulus =

1st Technique = Static Fat.
$\% \mathrm{~B}_{2} \mathrm{O}_{3}=$

$\% \mathrm{~K}_{2} \mathrm{O}=.632$

Other $\%=$

Other $\%=$
$\% \mathrm{CaO}=7.43$

$\% \mathrm{PbO}=$

$\% \mathrm{MgO}=5.91$

$\% \mathrm{SiO}_{2}=71.3$

$K_{\mathrm{IC}}=$

$K_{\mathrm{IC}}=$

$n=13$

Environment $=\mathrm{H}_{2} \mathrm{O}$

Reference $=$ MOULD,R.E.,SOUTHWICK,R.D.,JACTAW,42,542-47,582-92,1959

Comments $=$ Surface condition- abraded
Gamma $=$
Gamma $=$

\section{PRO67}

Specific Material =

Manufacturer $=$

$\% \mathrm{Al}_{2} \mathrm{O}_{3}=$

$\% \mathrm{Na}_{2} \mathrm{O}=$

Other Formula $=$

Other Formula $=$

Young's Modulus =

1st Technique $=$ Static Fat.

$\% \mathrm{~B}_{2} \mathrm{O}_{3}=$

$\% \mathrm{~K}_{2} \mathrm{O}=$

Other \% =

Other $\%=$
$\% \mathrm{CaO}=$

$\% \mathrm{PbO}=$

$K_{\text {IC }}=$

$K_{\mathrm{IC}}=$

2nd Technique $=$

$n=19.2$

Environment $=$ air, $50 \% \mathrm{RH}$

Reference = PROCTER,B.A.,Proc. Roy. Soc. Lond.,Ser. A,297,534-57,1967

Comments $=$ Surface condition- as-drawn fiber 
Specific Material =

Manufacturer $=$

$\% \mathrm{Al}_{2} \mathrm{O}_{3}=$

$\% \mathrm{Na}_{2} \mathrm{O}=$

Other Formula $=$

Other Formula =

Young's Modulus =

1st Technique $=$ Dynamic Fat.

2nd Technique $=$

$n=27$

Environment $=$ air, $50 \% \mathrm{RH}$

Reference $=$ PROCTER,B.A.,Proc. Roy. Soc. Lond.,Ser. A,297,534-57,1967

Comments $=$ Surface condition- as-drawn fiber

\section{RIT69}

Specific Material $=$

Manufacturer $=$

$\% \mathrm{Al}_{2} \mathrm{O}_{3}=1.16$

$\% \mathrm{Na}_{2} \mathrm{O}=13.4$

Other Formula =

Other Formula =

Young's Modulus =

1st Technique $=$ Dynamic Fat.

2nd Technique $=$

$n=13$

Environment $=$ air, $50 \% \mathrm{RH}$

Reference = RITTER,J.E.,Jr., Jour. of Appl. Phys., 40, 340-44, 1969

Comments $=$ Surface condition- acid etch and abraded

RIT71

Specific Material $=7740$

Manufacturer $=$ Corning Glass

$\% \mathrm{Al}_{2} \mathrm{O}_{3}=1.21$

$\% \mathrm{~B}_{2} \mathrm{O}_{3}=12.4$

$\% \mathrm{Na}_{2} \mathrm{O}=3.99$

Other Formula $=$

Other Formula =

$\% \mathrm{~K}_{2} \mathrm{O}=$

$\% \mathrm{CaO}=$

$\% \mathrm{PbO}=$

$\% \mathrm{CaO}=7.43$

$\% \mathrm{PbO}=$

$\% \mathrm{MgO}=5.91$

$\% \mathrm{SiO}_{2}=71.3$

Other $\%=$

$K_{\text {IC }}=$

$K_{\mathrm{IC}}=$

Other $\%=$

Other $\%=$

Young's Modulus =

1st Technique $=$ Dynamic Fat.

2nd Technique $=$

$n=27.4$

Environment $=$ air, $100 \% \mathrm{RH}$

Reference $=$ RITTER,J.E.,Jr.,SHERBOURNE,C.L.,JACTAW,54,601-05, 1971

Comments $=$ Surface condition- abraded

Specific Material =

Manufacturer $=$

$\% \mathrm{Al}_{2} \mathrm{O}_{3}=$

\% $\mathrm{Na}_{2} \mathrm{O}=$

Other Formula =

Other Formula =

Young's Modulus =

1st Technique $=$ Dynamic Fat.

$\% \mathrm{~B}_{2} \mathrm{O}_{3}=$

$\% \mathrm{~K}_{2} \mathrm{O}=$

$\% \mathrm{CaO}=$

$\% \mathrm{PbO}=$

$K_{\mathrm{IC}}=$

$K_{\mathrm{IC}}=$

Other $\%=$

Other $\%=$
Gamma =

Gamma $=$
Gamma $=$

Gamma $=$
$\% \mathrm{MgO}=$

$\% \mathrm{SiO}_{2}=82.3$

Gamma $=$

Gamma $=$

2nd Technique $=$

$n=37.8$

Environment $=$ air, $100 \% \mathrm{RH}$

Reference = RITTER,J.E.,Jr.,SHERBOURNE,C.L.,JACTAW,54,601-05, 1971

Comments $=$ Surface condition- abrade

$K_{\mathrm{IC}}=$

$K_{\mathrm{IC}}=$ 
Specific Material $=\mathrm{R} 6$

Manufacturer $=$ Owens-Illinois
$\% \mathrm{Al}_{2} \mathrm{O}_{3}=1.78$
$\% \mathrm{~B}_{2} \mathrm{O}_{3}=1.74$
$\% \mathrm{~K}_{2} \mathrm{O}=.645$
$\% \mathrm{CaO}=5.42$
$\% \mathrm{PbO}=$
$\% \mathrm{Na}_{2} \mathrm{O}=14.7$
Other $\%=.793$
Other Formula $=\mathrm{BaO}$
Other $\%=$
Other Formula $=$
Young's Modulus =
1st Technique $=$ Static Fat.
2nd Technique $=$
$K_{\mathrm{IC}}=$
$K_{\mathrm{IC}}=$
$n=32$
Environment $=$ air, $50 \% \mathrm{RH}$
Reference $=$ RITTER,J.E.,Jr.,SHERBOURNE,C.L.,JACTAW,54,601-05, 1971
Comments $=$ Surface condition- acid etch

$\% \mathrm{MgO}=6.03$

$\% \mathrm{SiO}_{2}=68.8$

Gamma $=$

\section{RIT73}

Specific Material $=7740$

Manufacturer $=$ Corning Glass

$\% \mathrm{Al}_{2} \mathrm{O}_{3}=1.21$

$\% \mathrm{~B}_{2} \mathrm{O}_{3}=12.4$

$\% \mathrm{~K}_{2} \mathrm{O}=$

$\% \mathrm{CaO}=$

$\% \mathrm{PbO}=$

Gamma $=$

$\% \mathrm{Na}_{2} \mathrm{O}=3.99$

Other Formula =

Other $\%=$

Other Formula =

Other $\%=$

Young's Modulus =

1st Technique $=$ Dynamic Fat.

2nd Technique $=$

$n=16$

Environment $=$ air, $100 \% \mathrm{RH}$

Reference = RITTER,J.E.,Jr.,MANTHURUTHIL,J.,Glass Tech., 14,60-64,1973

Comments $=$ Surface condition- abraded

Specific Material $=7740$

Manufacturer $=$ Corning Glass

$\% \mathrm{Al}_{2} \mathrm{O}_{3}=1.21$

$\% \mathrm{~B}_{2} \mathrm{O}_{3}=12.4$

$\% \mathrm{~K}_{2} \mathrm{O}=$

$\% \mathrm{CaO}=$

$\% \mathrm{MgO}=$

$\% \mathrm{Na}_{2} \mathrm{O}=3.99$

Other $\%=$

$\% \mathrm{PbO}=$

$\% \mathrm{MgO}=$

$\% \mathrm{SiO}_{2}=82.3$

Other Formula =

Other Formula =

Other $\%=$

$K_{\text {IC }}=$

$K_{\mathrm{IC}}=$

Gamma $=$

Gamma $=$

Young's Modulus =

\section{RIT75}

1st Technique $=$ Dynamic Fat.

2nd Technique $=$

$n=22$

Environment $=$ air, $100 \% \mathrm{RH}$

Reference $=$ RITTER,J.E.,Jr.,MANTHURUTHIL,J.,Glass Tech., 14,60-64,1973

Comments $=$ Surface condition- acid polished

Specific Material = R6

Manufacturer $=$ Owens-Illinois

$\% \mathrm{Al}_{2} \mathrm{O}_{3}=1.78 \quad \% \mathrm{~B}_{2} \mathrm{O}_{3}=1.74$

$\% \mathrm{Na}_{2} \mathrm{O}=14.7 \quad \% \mathrm{~K}_{2} \mathrm{O}=.645$

Other Formula $=\mathrm{BaO} \quad$ Other $\%=.793$

Other Formula =

Other $\%=$

$K_{1 \mathrm{C}}=$

$K_{\mathrm{IC}}=$

Young's Modulus =

1st Technique $=$ Dynamic Fat.

2nd Technique $=$

$n=19.5$

Environment $=6 \mathrm{~N} \mathrm{NaOH}$

Reference = RITTER,J.E.,Jr.,LAPORTE,R.P., JACTAW, 58, 265-67, 1975

Comments $=$ Surface condition- abraded

$$
\begin{array}{ll}
\% \mathrm{CaO}=5.42 & \% \mathrm{MgO}=6.03 \\
\% \mathrm{PbO}= & \% \mathrm{SiO}_{2}=68.8
\end{array}
$$

$K_{\mathrm{IC}}=$

$K_{\mathrm{IC}}=$

Gamma $=$

Gamma $=$ 
Specific Material $=\mathrm{R} 6$

Manufacturer $=$ Owens-Illinois
$\% \mathrm{Al}_{2} \mathrm{O}_{3}=1.78$
$\% \mathrm{~B}_{2} \mathrm{O}_{3}=1.74$
$\% \mathrm{CaO}=5.42$
$\% \mathrm{Na}_{2} \mathrm{O}=14.7$
$\% \mathrm{~K}_{2} \mathrm{O}=.645$
$\% \mathrm{PbO}=$
Other Formula $=\mathrm{BaO}$
Other $\%=.793$
Other Formula =
Other $\%=$
Young's Modulus =
1st Technique $=$ Dynamic Fat.
$K_{\mathrm{IC}}=$
Gamma $=$
2nd Technique $=$
$K_{\text {IC }}=$
Gamma $=$

$\% \mathrm{MgO}=6.03$

$\% \mathrm{SiO}_{2}=68.8$

$n=13$

Environment $=\mathrm{H}_{2} \mathrm{O}$

Reference = RITTER,J.E.,Jr.,LAPORTE,R.P., JACTAW, 58, 265-67, 1975

Comments $=$ Surface condition- abraded

Specific Material $=\mathrm{R} 6$

Manufacturer = Owens-Illinois
$\% \mathrm{Al}_{2} \mathrm{O}_{3}=1.78$
$\% \mathrm{~B}_{2} \mathrm{O}_{3}=1.74$
$\% \mathrm{CaO}=5.42$
$\% \mathrm{Na}_{2} \mathrm{O}=14.7$
$\% \mathrm{~K}_{2} \mathrm{O}=.645$
$\% \mathrm{PbO}=$
Other Formula $=\mathrm{BaO}$
Other $\%=.793$
Other Formula =
Other $\%=$
Young's Modulus =
1st Technique $=$ Dynamic Fat.
$K_{\mathrm{IC}}=$
Gamma $=$
2nd Technique $=$
$K_{\mathrm{IC}}=$
Gamma $=$

$\% \mathrm{MgO}=6.03$

$\% \mathrm{SiO}_{2}=68.8$

$n=25.1$

Environment $=6 \mathrm{~N} \mathrm{HCl}$

Reference $=$ RITTER,J.E.,Jr.,LAPORTE,R.P., JACTAW, 58, 265-67, 1975

Comments $=$ Surface condition- abraded

Specific Material $=\mathrm{R} 6$

Manufacturer $=$ Owens-Illinois
$\% \mathrm{Al}_{2} \mathrm{O}_{3}=1.78$
$\% \mathrm{~B}_{2} \mathrm{O}_{3}=1.74$
$\% \mathrm{CaO}=5.42$
$\% \mathrm{PbO}=$
$\% \mathrm{Na}_{2} \mathrm{O}=14.7$
$\% \mathrm{~K}_{2} \mathrm{O}=.645$
Other Formula $=\mathrm{BaO}$
Other $\%=.793$
Other Formula =
Other $\%=$
Young's Modulus =
1st Technique $=$ Dynamic Fat.
2nd Technique $=$
Gamma $=$
Gamma $=$

$\% \mathrm{MgO}=6.03$

$\% \mathrm{SiO}_{2}=68.8$

$n=19.3$

Environment $=6 \mathrm{~N} \mathrm{NaOH}$

Reference = RITTER,J.E.,Jr.,LAPORTE,R.P., JACTAW, 58, 265-67, 1975

Comments $=$ Surface condition- acid polished

Specific Material $=\mathrm{R} 6$

Manufacturer $=$ Owens-Illinois

$\% \mathrm{Al}_{2} \mathrm{O}_{3}=1.78$

$\% \mathrm{Na}_{2} \mathrm{O}=14.7$

$\% \mathrm{~B}_{2} \mathrm{O}_{3}=1.74$

$\% \mathrm{~K}_{2} \mathrm{O}=.645$

Other Formula $=\mathrm{BaO}$

Other Formula =

Other $\%=.793$

Other $\%=$

Young's Modulus =

1st Technique = Dynamic Fat.

2nd Technique $=$

$n=16.9$

Environment $=\mathrm{H}_{2} \mathrm{O}$

Reference = RITTER,J.E.,Jr.,LAPORTE,R.P., JACTAW, 58, 265-67, 1975

Comments $=$ Surface condition- acid polished

$K_{\mathrm{IC}}=$

$K_{\mathrm{IC}}=$

$\% \mathrm{MgO}=6.03$

$\% \mathrm{PbO}=$

$\% \mathrm{SiO}_{2}=68.8$

Gamma $=$

Gamma $=$ 
Specific Material $=\mathrm{R} 6$

Manufacturer $=$ Owens-Illinois

$\% \mathrm{Al}_{2} \mathrm{O}_{3}=1.78 \quad \% \mathrm{~B}_{2} \mathrm{O}_{3}=1.74$

$\% \mathrm{CaO}=5.42$

$\% \mathrm{PbO}=$

$\% \mathrm{MgO}=6.03$

$\% \mathrm{Na}_{2} \mathrm{O}=14.7$

$\% \mathrm{~K}_{2} \mathrm{O}=.645$

$\% \mathrm{SiO}_{2}=68.8$

Other Formula $=\mathrm{BaO}$

Other $\%=.793$

Other Formula $=$

Other $\%=$

Young's Modulus =

1st Technique $=$ Dynamic Fat.

2nd Technique $=$

$K_{\mathrm{IC}}=$

Gamma $=$

$K_{\mathrm{IC}}=$

Gamma $=$

$n=17.8$

Environment $=6 \mathrm{~N} \mathrm{HCl}$

Reference = RITTER,J.E.,Jr.,LAPORTE,R.P., JACTAW, 58, 265-67, 1975

Comments $=$ Surface condition- acid polished

Specific Material $=7740$

Manufacturer $=$ Corning Glass

$\% \mathrm{Al}_{2} \mathrm{O}_{3}=1.21$

$\% \mathrm{~B}_{2} \mathrm{O}_{3}=12.4$

$\% \mathrm{CaO}=$

$\% \mathrm{PbO}=$

$\% \mathrm{MgO}=$

$\% \mathrm{Na}_{2} \mathrm{O}=3.99$

$\% \mathrm{~K}_{2} \mathrm{O}=$

Other $\%=$

Other Formula =

Other $\%=$

$\% \mathrm{SiO}_{2}=82.3$

Young's Modulus =

lst Technique $=$ Dynamic Fat.

2nd Technique $=$

$n=22.6$

Environment $=6 \mathrm{~N} \mathrm{NaOH}$

Reference $=$ RITTER,J.E.,Jr.,LAPORTE,R.P., JACTAW, 58, 265-67, 1975

Comments $=$ Surface condition- abraded

Specific Material $=7740$

Manufacturer $=$ Corning Glass

$\% \mathrm{Al}_{2} \mathrm{O}_{3}=1.21$

$\% \mathrm{Na}_{2} \mathrm{O}=3.99$

\% $\mathrm{B}_{2} \mathrm{O}_{3}=12.4$

$\% \mathrm{CaO}=$

$K_{\text {IC }}=$

Gamma $=$

$K_{\text {IC }}=$

Gamma $=$

Other Formula =

$\% \mathrm{~K}_{2} \mathrm{O}=$

$\% \mathrm{PbO}=$

$\% \mathrm{MgO}=$

Other $\%=$

Other Formula =

Other $\%=$

$\% \mathrm{SiO}_{2}=82.3$

Young's Modulus =

1st Technique $=$ Dynamic Fat.

$K_{\mathrm{IC}}=$

Gamma $=$

2nd Technique $=$

$K_{\text {IC }}=$

Gamma $=$

$n=35.1$

Environment $=\mathrm{H}_{2} \mathrm{O}$

Reference = RITTER,J.E.,Jr.,LAPORTE,R.P., JACTAW, 58, 265-67, 1975

Comments $=$ Surface condition- abraded

Specific Material $=7740$

Manufacturer $=$ Corning Glass

$\% \mathrm{Al}_{2} \mathrm{O}_{3}=1.21 \quad \% \mathrm{~B}_{2} \mathrm{O}_{3}=12.4$

$\% \mathrm{~K}_{2} \mathrm{O}=$

$\% \mathrm{CaO}=$

$\% \mathrm{PbO}=$

$\% \mathrm{MgO}=$

$\% \mathrm{Na}_{2} \mathrm{O}=3.99$

Other $\%=$

Other Formula =

Other $\%=$

$\% \mathrm{SiO}_{2}=82.3$

Other Formula =

Young's Modulus =

1st Technique = Dynamic Fat.

$K_{\text {IC }}=$

$K_{\mathrm{IC}}=$

Gamma $=$

2nd Technique $=$

$n=26.9$

Environment $=6 \mathrm{~N} \mathrm{HCl}$

Reference = RITTER,J.E.,Jr.,LAPORTE,R.P., JACTAW, 58, 265-67, 1975

Comments $=$ Surface condition- abraded 
Specific Material $=7740$

Manufacturer $=$ Corning Glass

$\begin{array}{ll}\% \mathrm{Al}_{2} \mathrm{O}_{3}=1.21 & \% \mathrm{~B}_{2} \mathrm{O}_{3}=12.4 \\ \% \mathrm{Na}_{2} \mathrm{O}=3.99 & \% \mathrm{~K}_{2} \mathrm{O}= \\ \text { Other Formula }= & \text { Other \%= } \\ \text { Other Formula }= & \text { Other \% }=\end{array}$

$\% \mathrm{CaO}=$

$\% \mathrm{PbO}=$

$K_{\mathrm{IC}}=$

$K_{\mathrm{IC}}=$

2nd Technique $=$

$n=21.1$

Environment $=6 \mathrm{~N} \mathrm{NaOH}$

Reference = RITTER,J.E.,Jr.,LAPORTE,R.P., JACTAW, 58, 265-67, 1975

Comments $=$ Surface condition- acid polished

Specific Material $=7740$

Manufacturer $=$ Corning Glass

$\% \mathrm{Al}_{2} \mathrm{O}_{3}=1.21$

$\% \mathrm{Na}_{2} \mathrm{O}=3.99$

Other Formula =

$\% \mathrm{~B}_{2} \mathrm{O}_{3}=12.4$

$\% \mathrm{~K}_{2} \mathrm{O}=$

Other $\%=$

$\% \mathrm{CaO}=$

$\% \mathrm{PbO}=$

Other $\%=$

Other Formula =

Young's Modulus =

1st Technique $=$ Dynamic Fat.

2nd Technique $=$

$K_{\mathrm{IC}}=$

$K_{\mathrm{IC}}=$

$n=39.8$

Environment $=\mathrm{H}_{2} \mathrm{O}$

Reference = RITTER,J.E.,Jr.,LAPORTE,R.P., JACTAW, 58, 265-67, 1975

Comments $=$ Surface condition- acid polished

Specific Material $=7740$

Manufacturer $=$ Corning Glass

$\% \mathrm{Al}_{2} \mathrm{O}_{3}=1.21$

$\% \mathrm{~B}_{2} \mathrm{O}_{3}=12.4$

$\% \mathrm{Na}_{2} \mathrm{O}=3.99$

Other Formula =

Other Formula =

$\% \mathrm{~K}_{2} \mathrm{O}=$

Other $\%=$

Young's Modulus =

Other $\%=$

$\% \mathrm{CaO}=$

$\% \mathrm{PbO}=$

$\% \mathrm{MgO}=$

$\% \mathrm{SiO}_{2}=82.3$

1st Technique $=$ Dynamic Fat.

2nd Technique $=$

$n=64.9$

Environment $=6 \mathrm{~N} \mathrm{HCl}$

Reference = RITTER,J.E.,Jr.,LAPORTE,R.P., JACTAW, 58, 265-67, 1975

Comments $=$ Surface condition- acid polished

Specific Material $=7740$

Manufacturer $=$ Corning Glass

$\% \mathrm{Al}_{2} \mathrm{O}_{3}=1.21$

$\% \mathrm{Na}_{2} \mathrm{O}=3.99$

Other Formula =

Other Formula =

Young's Modulus =

1st Technique $=\mathrm{DCB}$

2nd Technique $=$

$n=34.1$

$\% \mathrm{~B}_{2} \mathrm{O}_{3}=12.4$

$\% \mathrm{~K}_{2} \mathrm{O}=$

Other \% =

Other $\%=$

$K_{\text {IC }}=$

$K_{\mathrm{IC}}=$

Gamma $=$

Gamma $=$
Gamma =

Gamma $=$
Gamma = Gamma $=$

Environment $=\mathrm{H}_{2} \mathrm{O}$

Reference = RITTER,J.E.,Jr.,LAPORTE,R.P., JACTAW, 58, 265-67, 1975

Comments $=$ 
Specific Material $=7740$

Manufacturer $=$ Corning Glass
$\% \mathrm{Al}_{2} \mathrm{O}_{3}=1.21$
$\% \mathrm{~B}_{2} \mathrm{O}_{3}=12.4$
$\% \quad \mathrm{Na}_{2} \mathrm{O}=3.99$
$\% \mathrm{~K}_{2} \mathrm{O}=$
Other Formula =
Other $\%=$
Other Formula =
Other $\%=$

$\% \mathrm{CaO}=$

$\% \mathrm{MgO}=$

$\% \mathrm{PbO}=$

$\% \mathrm{SiO}_{2}=82.3$

Young's Modulus =

1st Technique $=\mathrm{DCB}$

$K_{\mathrm{IC}}=$

Gamma $=$

2nd Technique $=$

$K_{\mathrm{IC}}=$

Gamma =

$n=22.7$

Environment $=6 \mathrm{~N} \mathrm{NaOH}$

Reference = RITTER,J.E.,Jr.,LAPORTE,R.P., JACTAW, 58, 265-67, 1975

Comments $=$

Specific Material $=7740$

Manufacturer $=$ Corning Glass

$\begin{array}{ll}\% \mathrm{Al}_{2} \mathrm{O}_{3}=1.21 & \% \mathrm{~B}_{2} \mathrm{O}_{3}=12.4 \\ \% \mathrm{Na}_{2} \mathrm{O}=3.99 & \% \mathrm{~K}_{2} \mathrm{O}= \\ \text { Other Formula }= & \text { Other } \%= \\ \text { Other Formula }= & \text { Other } \%=\end{array}$

$\% \mathrm{CaO}=$

$\% \mathrm{MgO}=$

$\% \mathrm{PbO}=$

$\% \mathrm{SiO}_{2}=82.3$

Young's Modulus =

1st Technique $=\mathrm{DCB}$

2nd Technique $=$

$K_{\mathrm{IC}}=$

Gamma $=$

$n=57$

$K_{\mathrm{IC}}=$

Gamma $=$

Environment $=6 \mathrm{~N} \mathrm{HCl}$

Reference = RITTER,J.E.,Jr.,LAPORTE,R.P., JACTAW, 58, 265-67, 1975

Comments $=$

SHI79

Specific Material =

Manufacturer $=$

$\% \mathrm{Al}_{2} \mathrm{O}_{3}=$

$\% \mathrm{~B}_{2} \mathrm{O}_{3}=30$

$\% \mathrm{Na}_{2} \mathrm{O}=$

$\% \mathrm{~K}_{2} \mathrm{O}=$

$\% \mathrm{CaO}=$

$\% \mathrm{PbO}=70$

$\% \mathrm{MgO}=$

Other $\%=$

$\% \mathrm{SiO}_{2}=$

Other Formula =

Other Formula =

Other $\%=$

Young's Modulus $=4.33 E 4$

1st Technique $=$ Controlled Flaw

$K_{\mathrm{IC}}=.32$

2nd Technique $=$

$K_{\mathrm{IC}}=$

Gamma $=1.16$

$n=$

Environment $=\mathrm{N}_{2}(1)$ at $-196 \mathrm{C}$

Reference $=$ SHINKAI,N.,Thesis by author at Penn. State Univ., 1979

Comments $=$

Specific Material =

Manufacturer $=$

$\begin{array}{ll}\% \mathrm{Al}_{2} \mathrm{O}_{3}= & \% \mathrm{~B}_{2} \mathrm{O}_{3}=30 \\ \% \mathrm{Na}_{2} \mathrm{O}= & \% \mathrm{~K}_{2} \mathrm{O}= \\ \text { Other Formula }= & \text { Other \%= } \\ \text { Other Formula }= & \text { Other } \%=\end{array}$

$\% \mathrm{CaO}=$

$\% \mathrm{MgO}=$

$\% \mathrm{PbO}=70 \quad \% \mathrm{SiO}_{2}=$

Young's Modulus $=4.33 E 4$

1st Technique $=$ Controlled Flaw

2nd Technique $=$

$K_{\mathrm{IC}}=.24$

Gamma $=.65$

$n=$

$K_{\mathrm{IC}}=$

Gamma $=$

Environment $=$ air

Reference $=$ SHINKAI,N.,Thesis by author at Penn. State Univ., 1979

Comments $=$ 
Specific Material =

Manufacturer $=$

$\% \mathrm{Al}_{2} \mathrm{O}_{3}=$

$\% \mathrm{Na}_{2} \mathrm{O}=$

Other Formula $=\mathrm{ZnO}$

Other Formula =

Young's Modulus

1st Technique $=$ Controlled Flaw

2nd Technique $=$

$n=$

Environment $=\mathrm{N}_{2}(1)$ at $-196 \mathrm{C}$

Reference $=$ SHINKAI,N., Thesis by author at Penn. State Univ., 1979

Comments

Specific Material $=$

Manufacturer $=$

$\% \mathrm{Al}_{2} \mathrm{O}_{3}=$

$\% \mathrm{~B}_{2} \mathrm{O}_{3}=30$

$\% \mathrm{Na}_{2} \mathrm{O}=$

$\% \mathrm{~K}_{2} \mathrm{O}=$

Other $\%=35$

Other Formula $=\mathrm{ZnO}$

Other Formula =

Young's Modulus $=6.1 E 4$

1st Technique $=$ Controlled Flaw

2nd Technique $=$

Other $\%=$

$K_{\mathrm{IC}}=.44$

$K_{\mathrm{IC}}=$
$\% \mathrm{CaO}=$

$\% \mathrm{PbO}=52.5$

$n=$

Environment $=\mathrm{N}_{2}(1)$ at $-196 \mathrm{C}$

Reference $=$ SHINKAI,N.,Thesis by author at Penn. State Univ., 1979

Comments $=$

Specific Material $=$

Manufacturer $=$

Gamma $=1.82$

Gamma $=$

$\% \mathrm{CaO}=$

$\% \mathrm{PbO}=35$

$K_{\mathrm{IC}}=.61$

$K_{\mathrm{IC}}=$

Gamma $=3.05$

Gamma $=$
$\% \mathrm{Al}_{2} \mathrm{O}_{3}=$

$\% \mathrm{~B}_{2} \mathrm{O}_{3}=30$
$\% \mathrm{~K}_{2} \mathrm{O}=$

Other Formula $=\mathrm{ZnO}$

Other Formula =

Young's Modulus $=6.1 E 4$

1st Technique $=$ Controlled Flaw

2nd Technique $=$

Other $\%=35$

Other $\%=$
$\% \mathrm{Na}_{2} \mathrm{O}=$

$\% \mathrm{CaO}=$

$\% \mathrm{PbO}=35$

$K_{\text {IC }}=.38$

$K_{\text {IC }}=$

$n=$

Environment $=$ air

Reference $=$ SHINKAI,N.,Thesis by author at Penn. State Univ., 1979

Comments $=$

Specific Material =

Manufacturer $=$

$\% \mathrm{Al}_{2} \mathrm{O}_{3}=$

$\% \mathrm{~B}_{2} \mathrm{O}_{3}=40$

$\% \mathrm{Na}_{2} \mathrm{O}=$

Other Formula =

Other Formula =

Young's Modulus $=5.55 E 4$

lst Technique $=$ Controlled Flaw

2nd Technique $=$

$\% \mathrm{~K}_{2} \mathrm{O}=$

Other $\%=$

Other $\%=$
$\% \mathrm{CaO}=$

$\% \mathrm{PbO}=60$

$K_{\mathrm{IC}}=.37$

$K_{\mathrm{IC}}=$
$\% \mathrm{MgO}=$

$\% \mathrm{SiO}_{2}=$

$n=$

Environment $=\mathrm{N}_{2}(\mathrm{l})$ at $-196 \mathrm{C}$

Reference $=$ SHINKAI,N.,Thesis by author at Penn. State Univ., 1979

Comments $=$ 
Specific Material $=$

Manufacturer $=$
$\% \mathrm{Al}_{2} \mathrm{O}_{3}=$
$\% \mathrm{~B}_{2} \mathrm{O}_{3}=40$
$\% \mathrm{CaO}=$
$\% \mathrm{Na}_{2} \mathrm{O}=$
$\% \mathrm{~K}_{2} \mathrm{O}=$
$\% \mathrm{PbO}=60$
$\% \mathrm{MgO}=$
Other Formula =
Other $\%=$
Other Formula =
Other $\%=$
$\% \mathrm{SiO}_{2}=$
Young's Modulus $=5.55 E 4$
lst Technique $=$ Controlled Flaw
$K_{\mathrm{IC}}=.36$
Gamma $=1.17$
2nd Technique $=$
$K_{\text {IC }}=$
Gamma $=$

$n=$

Environment $=$ air

Reference $=$ SHINKAI,N.,Thesis by author at Penn. State Univ., 1979

Comments $=$

Specific Material =

Manufacturer $=$

$\% \mathrm{Al}_{2} \mathrm{O}_{3}=$

$\% \mathrm{~B}_{2} \mathrm{O}_{3}=30$

$\% \mathrm{CaO}=$

$\% \mathrm{Na}_{2} \mathrm{O}=$

$\% \mathrm{~K}_{2} \mathrm{O}=$

$\% \mathrm{PbO}=52.5$

$\% \mathrm{MgO}=$

Other Formula $=\mathrm{ZnO}$

Other $\%=17.5$

Other Formula =

Other $\%=$

Young's Modulus $=5.27 E 4$

lst Technique $=$ Controlled Flaw

$K_{\mathrm{IC}}=.29$

2nd Technique $=$

$K_{\mathrm{IC}}=$

$\mathrm{Gamma}=.78$

$n=$

Environment $=$ air

Reference $=$ SHINKAI,N., Thesis by author at Penn. State Univ., 1979

Comments $=$

Specific Material $=$

Manufacturer $=$
$\% \mathrm{Al}_{2} \mathrm{O}_{3}=$
$\% \mathrm{~B}_{2} \mathrm{O}_{3}=40$
$\% \mathrm{CaO}=$
$\% \mathrm{MgO}=$
$\% \mathrm{Na}_{2} \mathrm{O}=$
$\% \mathrm{~K}_{2} \mathrm{O}=$
$\% \mathrm{PbO}=45$
$\% \mathrm{SiO}_{2}=$
Other Formula $=\mathrm{ZnO} \quad$ Other $\%=15$
Other Formula =
Other $\%=$
Young's Modulus $=6.98 E 4$
lst Technique $=$ Controlled Flaw
$K_{\mathrm{IC}}=.48$
2nd Technique $=$
$K_{\mathrm{IC}}=$
Gamma $=1.66$
Gamma $=$

Gamma $=$

$n=$

Environment $=\mathrm{N}_{2}(1)$ at $-196 \mathrm{C}$

Reference $=$ SHINKAI,N., Thesis by author at Penn. State Univ., 1979

Comments $=$

Specific Material $=$

Manufacturer =

$\% \mathrm{Al}_{2} \mathrm{O}_{3}=$

$\% \mathrm{~B}_{2} \mathrm{O}_{3}=40$

$\% \mathrm{CaO}=$

$\% \mathrm{MgO}=$

$\% \mathrm{Na}_{2} \mathrm{O}=$

$\% \mathrm{~K}_{2} \mathrm{O}=$

Other Formula $=\mathrm{ZnO}$

Other $\%=15$

$\% \mathrm{PbO}=45$

$\% \mathrm{SiO}_{2}=$

Other Formula =

Other $\%=$

Young's Modulus $=6.98 E 4$

lst Technique $=$ Controlled Flaw

$K_{\mathrm{IC}}=.43$

2nd Technique $=$

$K_{\mathrm{IC}}=$

Gamma $=1.30$

$n=$

Environment $=$ air

Reference $=$ SHINKAI,N.,Thesis by author at Penn. State Univ., 1979

Comments 
Specific Material =

Manufacturer $=$

$\% \mathrm{Al}_{2} \mathrm{O}_{3}=$

$\% \mathrm{Na}_{2} \mathrm{O}=$

Other Formula $=\mathrm{ZnO}$

Other Formula =

Young's Modulus = 7.47 E4

lst Technique $=$ Controlled Flaw

2nd Technique $=$

$n=$

Environment $=\mathrm{N}_{2}(1)$ at $-196 \mathrm{C}$

Reference $=$ SHINKAI,N.,Thesis by author at Penn. State Univ., 1979

Comments $=$

Specific Material =

Manufacturer $=$

$\% \mathrm{Al}_{2} \mathrm{O}_{3}=$

$\% \mathrm{~B}_{2} \mathrm{O}_{3}=40$

$\% \mathrm{Na}_{2} \mathrm{O}=$

$\% \mathrm{~K}_{2} \mathrm{O}=$

Other Formula $=\mathrm{ZnO}$

Other $\%=30$

$K_{\mathrm{IC}}=.61$

$K_{\mathrm{IC}}=$

$\% \mathrm{CaO}=$

$\% \mathrm{PbO}=30$

$\% \mathrm{MgO}=$

$\% \mathrm{SiO}_{2}=$

Other $\%=30$

Other $\%=$

$\% \mathrm{CaO}=$

$\% \mathrm{PbO}=30$

$\% \mathrm{MgO}=$

$\% \mathrm{SiO}_{2}=$

Gamma $=$

Other Formula =

Other $\%=$

Young's Modulus $=7.47 E 4$

1st Technique $=$ Controlled Flaw

2nd Technique $=$

$n=$

Environment $=$ air

Reference $=$ SHINKAI,N.,Thesis by author at Penn. State Univ., 1979

Comments $=$

Specific Material $=$

Manufacturer $=$

$\% \mathrm{Al}_{2} \mathrm{O}_{3}=$

$\% \mathrm{~B}_{2} \mathrm{O}_{3}=40$

$\% \mathrm{CaO}=$

$\% \mathrm{Na}_{2} \mathrm{O}=$

$\% \mathrm{~K}_{2} \mathrm{O}=$

Other Formula $=\mathrm{ZnO}$

Other $\%=45$

$\% \mathrm{PbO}=15$

Gamma $=2.47$

Other Formula =

Young's Modulus $=7.9 \mathrm{E} 4$

1st Technique $=$ Controlled Flaw

Other $\%=$

$$
K_{\mathrm{IC}}=
$$

Gamma $=2.11$

Gamma $=$

2nd Technique $=$

$=.75$

$K_{\mathrm{IC}}=$

Gamma $=3.57$

$n=$

Environment $=\mathrm{N}_{2}(\mathrm{l})$ at $-196 \mathrm{C}$

Reference $=$ SHINKAI,N.,Thesis by author at Penn. State Univ., 1979

Comments $=$

Specific Material =

Manufacturer $=$

$\% \mathrm{Al}_{2} \mathrm{O}_{3}=$

$\% \mathrm{~B}_{2} \mathrm{O}_{3}=40$

$\% \mathrm{~K}_{2} \mathrm{O}=$

$\% \mathrm{Na}_{2} \mathrm{O}=$

Other $\%=45$

Other Formula $=\mathrm{ZnO}$

Other Formula =

Young's Modulus $=7.9 E 4$

1st Technique $=$ Controlled Flaw

2nd Technique $=$

$n=$

Environment $=$ air

Reference $=$ SHINKAI,N.,Thesis by author at Penn. State Univ., 1979

Comments $=$
Other $\%=$
$K_{\mathrm{IC}}=.61$

$K_{\mathrm{IC}}=$
$\% \mathrm{CaO}=$

$\% \mathrm{PbO}=15 \quad \% \mathrm{SiO}_{2}=$
$\% \mathrm{MgO}=$

$\% \mathrm{SiO}_{2}=$
Gamma $=2.32$

Gamma $=$ 
Specific Material $=$

Manufacturer $=$

$\% \mathrm{Al}_{2} \mathrm{O}_{3}=$

$\% \mathrm{Na}_{2} \mathrm{O}=$

Other Formula $=\mathrm{ZnO}$

Other Formula =

Young's Modulus $=8.88 E 4$

1st Technique $=$ Controlled Flaw

2nd Technique $=$

$n=$

Environment $=\mathrm{N}_{2}(\mathrm{l})$ at $-196 \mathrm{C}$

Reference $=$ SHINKAI,N.,Thesis by author at Penn. State Univ., 1979

Comments $=$

Specific Material $=$

Manufacturer $=$

$\% \mathrm{Al}_{2} \mathrm{O}_{3}=$

$\% \mathrm{Na}_{2} \mathrm{O}=$

$\% \mathrm{~B}_{2} \mathrm{O}_{3}=40$

$\% \mathrm{~K}_{2} \mathrm{O}=$

Other Formula $=\mathrm{ZnO}$

Other Formula =
Other $\%=60$

$\% \mathrm{CaO}=$

$\% \mathrm{PbO}=$

Other $\%=$
$\% \mathrm{CaO}=$

$\% \mathrm{PbO}=$

$K_{\mathrm{IC}}=.89$

$K_{\mathrm{IC}}=$

Gamma $=4.45$

Gamma $=$

Young's Modulus $=8.88 E 4$

1st Technique $=$ Controlled Flaw

2nd Technique $=$

$K_{\mathrm{IC}}=.80$

$n=$

Gamma $=3.64$

Environment $=$ air

Reference $=$ SHINKAI,N.,Thesis by author at Penn. State Univ., 1979

Comments $=$

Specific Material =

Manufacturer =

$\% \mathrm{Al}_{2} \mathrm{O}_{3}=$

$\% \mathrm{~B}_{2} \mathrm{O}_{3}=50$

$\% \mathrm{Na}_{2} \mathrm{O}=$

$\% \mathrm{~K}_{2} \mathrm{O}=$

$\% \mathrm{CaO}=$

Other $\%=$

Other Formula =

Other $\%=$

$\% \mathrm{PbO}=50$

$\% \mathrm{MgO}=$

$\% \mathrm{SiO}_{2}=$

Other Formula =

Young's Modulus $=6.12 E 4$

1st Technique $=$ Controlled Flaw

2nd Technique $=$

$K_{\mathrm{IC}}=.51$

$K_{\mathrm{IC}}=$

Gamma $=2.13$

$n=$

Environment $=\mathrm{N}_{2}(1)$ at $-196 \mathrm{C}$

Reference $=$ SHINKAI,N.,Thesis by author at Penn. State Univ., 1979

Comments $=$

Specific Material $=$

Manufacturer $=$

$\% \mathrm{Al}_{2} \mathrm{O}_{3}=$

$\% \mathrm{~B}_{2} \mathrm{O}_{3}=50$

$\% \mathrm{Na}_{2} \mathrm{O}=$

Other Formula $=$

Other Formula =

$\% \mathrm{~K}_{2} \mathrm{O}=$

$\% \mathrm{CaO}=$

$\% \mathrm{PbO}=50$

$\% \mathrm{MgO}=$

Other $\%=$

Other $\%=$

Gamma $=$

Young's Modulus $=6.12 E 4$

Ist Technique $=$ Controlled Flaw

$K_{\mathrm{IC}}=.40$

$K_{\mathrm{IC}}=$

Gamma $=1.33$

2nd Technique $=$

$$
K_{\mathrm{ic}}
$$

Environment $=$ air

Reference $=$ SHINKAI,N.,Thesis by author at Penn. State Univ., 1979

Comments $=$ 
Specific Material =

Manufacturer =

$\% \mathrm{Al}_{2} \mathrm{O}_{3}=$

$\% \mathrm{~B}_{2} \mathrm{O}_{3}=50$

$\% \mathrm{CaO}=$

$\% \mathrm{PbO}=37.5$

$\% \mathrm{MgO}=$

$\% \mathrm{~K}_{2} \mathrm{O}=$

$\% \mathrm{Na}_{2} \mathrm{O}=$

Other $\%=12.5$

Other Formula $=\mathrm{ZnO}$

Other $\%=$

Other Formula =

\section{4}

1st Technique $=$ Controlled Flaw

2nd Technique $=$

$n=$

Environment $=\mathrm{N}_{2}(\mathrm{l})$ at $-196 \mathrm{C}$

Reference $=$ SHINKAI,N.,Thesis by author at Penn. State Univ., 1979

Comments $=$

Specific Material =

Manufacturer =

$\% \mathrm{Al}_{2} \mathrm{O}_{3}=$

$\% \mathrm{~B}_{2} \mathrm{O}_{3}=50$

$\% \mathrm{CaO}=$

$\% \mathrm{PbO}=37.5$

$\% \mathrm{MgO}=$

$\% \mathrm{~K}_{2} \mathrm{O}=$

$K_{\mathrm{IC}}=.63$

$K_{\mathrm{IC}}=$

Gamma $=2.74$

Gamma $=$
Other Formula $=\mathrm{ZnO}$

Other Formula =

$\%=12.5$

Other $\%=$

Young's Modulus $=7.13 E 4$

1st Technique $=$ Controlled Flaw

2nd Technique $=$

$n=$

Environment $=$ air

Reference $=$ SHINKAI,N., Thesis by author at Penn. State Univ., 1979

Comments $=$

\section{Specific Material $=$}

Manufacturer $=$

$\% \mathrm{Al}_{2} \mathrm{O}_{3}=$

$\% \mathrm{Na}_{2} \mathrm{O}=$

$\% \mathrm{~B}_{2} \mathrm{O}_{3}=50$

$\% \mathrm{~K}_{2} \mathrm{O}=$

$K_{\mathrm{IC}}=.46$

$K_{\mathrm{IC}}=$

Other Formula $=\mathrm{ZnO}$

Other $\%=25$

Other Formula =

Other $\%=$

Young's Modulus $=8.08 \mathrm{E4}$

1st Technique $=$ Controlled Flaw

2nd Technique $=$

$n=$

Environment $=\mathrm{N}_{2}(\mathrm{l})$ at $-196 \mathrm{C}$

Reference $=$ SHINKAI,N.,Thesis by author at Penn. State Univ., 1979

Comments $=$

Specific Material =

Manufacturer $=$

$\% \mathrm{Al}_{2} \mathrm{O}_{3}=$

$\% \mathrm{Na}_{2} \mathrm{O}=$

$\% \mathrm{~K}_{2} \mathrm{O}=$

$K_{\mathrm{IC}}=.77$

$K_{\mathrm{IC}}=$

$\% \mathrm{CaO}=$

$\% \mathrm{PbO}=25$

Gamma $=1.48$

Gamma $=$

Other Formula $=\mathrm{ZnO}$

Other Formula =

Young's Modulus $=8.08 E 4$

1st Technique $=$ Controlled Flaw

2nd Technique $=$

$n=$

Environment $=$ air

Reference $=$ SHINKAI,N.,Thesis by author at Penn. State Univ., 1979

Comments $=$
$\% \mathrm{~B}_{2} \mathrm{O}_{3}=50$

Other $\%=25$

$$
\% \mathrm{CaO}=
$$

$\% \mathrm{PbO}=25$

$K_{\mathrm{IC}}=.64$

$K_{\text {IC }}=$
$\% \mathrm{MgO}=$

$\% \mathrm{SiO}_{2}=$

Gamma $=3.70$

Gamma $=$
\% $\mathrm{MgO}=$

$\% \mathrm{SiO}_{2}=$

Gamma $=2.54$

Gamma $=$ 
Specific Material $=$

Manufacturer $=$

$\% \mathrm{Al}_{2} \mathrm{O}_{3}=$

$\% \mathrm{Na}_{2} \mathrm{O}=$

Other Formula $=\mathrm{ZnO}$

Other Formula =

Young's Modulus $=8.26 E 4$

lst Technique $=$ Controlled Flaw

2nd Technique $=$

$n=$

Environment $=\mathrm{N}_{2}(1)$ at $-196 \mathrm{C}$

Reference $=$ SHINKAI,N.,Thesis by author at Penn. State Univ., 1979

Comments $=$

Specific Material =

Manufacturer $=$

$\% \mathrm{Al}_{2} \mathrm{O}_{3}=$

$\% \mathrm{Na}_{2} \mathrm{O}=$

$\% \mathrm{~B}_{2} \mathrm{O}_{3}=50$

$\% \mathrm{~K}_{2} \mathrm{O}=$

Other Formula $=\mathrm{ZnO}$

Other $\%=37.5$

Other Formula =

Other $\%=$

$K_{\text {IC }}=.89$

$K_{\mathrm{IC}}=$
$\% \mathrm{CaO}=$

$\% \mathrm{PbO}=12.5 \quad \% \mathrm{SiO}_{2}=$

Young's Modulus $=8.26 E 4$

1 st Technique $=$ Controlled Flaw

Gamma $=4.80$

Gamma =

2nd Technique $=$

$K_{\mathrm{IC}}=.71$

$\% \mathrm{CaO}=$

$\% \mathrm{PbO}=12.5$

$\% \mathrm{MgO}=$

$\% \mathrm{SiO}_{2}=$

$n=$

Environment $=$ air

Reference $=$ SHINKAI,N.,Thesis by author at Penn. State Univ., 1979

Comments $=$

Specific Material =

Manufacturer $=$

$\% \mathrm{Al}_{2} \mathrm{O}_{3}=$

$\% \mathrm{~B}_{2} \mathrm{O}_{3}=50$

$\% \mathrm{Na}_{2} \mathrm{O}=$

$\% \mathrm{~K}_{2} \mathrm{O}=$

$\% \mathrm{CaO}=$

$\% \mathrm{PbO}=$

Gamma $=3.09$

Other Formula $=\mathrm{ZnO}$

Other $\%=50$

Other Formula =

Other $\%=$

Young's Modulus $=9.73 E 4$

1st Technique $=$ Controlled Flaw

$K_{\mathrm{IC}}=.88$

2nd Technique $=$

$K_{\mathrm{IC}}=$

Gamma $=$

$n=$

Environment $=\mathrm{N}_{2}(1)$ at $-196 \mathrm{C}$

Reference $=$ SHINKAI,N.,Thesis by author at Penn. State Univ., 1979

Comments $=$

Specific Material $=$

Manufacturer $=$

$\% \mathrm{Al}_{2} \mathrm{O}_{3}=$

$\% \mathrm{~B}_{2} \mathrm{O}_{3}=50$

$\% \mathrm{Na}_{2} \mathrm{O}=$

$\% \mathrm{~K}_{2} \mathrm{O}=$

$\% \mathrm{CaO}=$

$\% \mathrm{PbO}=$

$\% \mathrm{MgO}=$

$\% \mathrm{SiO}_{2}=$

Other Formula $=\mathrm{ZnO} \quad$ Other $\%=50$

Other Formula $=$

Other $\%=$

Young's Modulus $=9.73 E 4$

lst Technique $=$ Controlled Flaw

$K_{\mathrm{IC}}=.83$

$K_{\mathrm{IC}}=$

Gamma $=3.98$

Gamma $=$

2nd Technique $=$

$n=$

Environment $=$ air

Reference $=$ SHINKAI,N.,Thesis by author at Penn. State Univ., 1979

Comments $=$

Gamma $=3.57$

Gamma =

$\% \mathrm{MgO}=$

$\% \mathrm{SiO}_{2}=$ 
Specific Material =

Manufacturer $=$
$\% \mathrm{Al}_{2} \mathrm{O}_{3}=$
$\% \mathrm{~B}_{2} \mathrm{O}_{3}=60$
$\% \mathrm{CaO}=$
$\% \mathrm{MgO}=$
$\% \mathrm{Na}_{2} \mathrm{O}=$
$\% \mathrm{~K}_{2} \mathrm{O}=$
$\% \mathrm{PbO}=40$
$\% \mathrm{SiO}_{2}=$
Other Formula =
Other $\%=$
Other Formula =
Other $\%=$
Young's Modulus $=6.37 E 4$
1st Technique $=$ Controlled Flaw
$K_{\mathrm{IC}}=.80$
Gamma $=4.99$
2nd Technique $=$
$K_{\mathrm{IC}}=$
Gamma $=$

$n=$

Environment $=\mathrm{N}_{2}(1)$ at $-196 \mathrm{C}$

Reference $=$ SHINKAI,N., Thesis by author at Penn. State Univ., 1979

Comments $=$

Specific Material $=$

Manufacturer $=$

$\% \mathrm{Al}_{2} \mathrm{O}_{3}=$

$\% \mathrm{Na}_{2} \mathrm{O}=$

$\% \mathrm{~B}_{2} \mathrm{O}_{3}=60$

$\% \mathrm{~K}_{2} \mathrm{O}=$

$\% \mathrm{CaO}=$

$\% \mathrm{PbO}=40$

$\% \mathrm{MgO}=$

Other $\%=$

$\% \mathrm{SiO}_{2}=$

Other Formula =

Other $\%=$

Other Formula =

Young's Modulus $=6.37 E 4$

1st Technique $=$ Controlled Flaw

$K_{\mathrm{IC}}=.50$

Gamma $=1.92$

2nd Technique $=$

$K_{\mathrm{IC}}=$

Gamma $=$

$n=$

Environment $=$ air

Reference $=$ SHINKAI,N., Thesis by author at Penn. State Univ., 1979

Comments $=$

Specific Material =

Manufacturer $=$

$\% \mathrm{Al}_{2} \mathrm{O}_{3}=$

$\% \mathrm{~B}_{2} \mathrm{O}_{3}=60$

$\% \mathrm{Na}_{2} \mathrm{O}=$

$\% \mathrm{~K}_{2} \mathrm{O}=$

Other Formula $=\mathrm{ZnO}$

Other $\%=10$

$\% \mathrm{CaO}=$

$\% \mathrm{PbO}=30$

$\% \mathrm{MgO}=$

Other Formula =

Other $\%=$

$\% \mathrm{SiO}_{2}=$

Young's Modulus $=8.35 E 4$

1 st Technique $=$ Controlled Flaw

2nd Technique $=$

$K_{\mathrm{IC}}=.90$

$K_{\text {IC }}=$

Gamma $=4.89$

$n=$

Environment $=\mathrm{N}_{2}(1)$ at $-196 \mathrm{C}$

Reference $=$ SHINKAI,N., Thesis by author at Penn. State Univ., 1979

Comments $=$

Specific Material $=$

Manufacturer $=$

$\% \mathrm{Al}_{2} \mathrm{O}_{3}=$

$\% \mathrm{~B}_{2} \mathrm{O}_{3}=60$

$\% \mathrm{Na}_{2} \mathrm{O}=$

$\% \mathrm{~K}_{2} \mathrm{O}=$

Other Formula $=\mathrm{ZnO}$

Other $\%=10$

$\% \mathrm{CaO}=$

$\% \mathrm{MgO}=$

Other Formula =

Other $\%=$

$\% \mathrm{PbO}=30$

$\% \mathrm{SiO}_{2}=$

Young's Modulus $=8.35 E 4$

1 st Technique $=$ Controlled Flaw

2nd Technique $=$

$K_{\mathrm{IC}}=.55$

Gamma $=1.79$

$n=$

$K_{\mathrm{IC}}=$

Gamma $=$

Environment $=$ air

Reference $=$ SHINKAI,N.,Thesis by author at Penn. State Univ., 1979

Comments $=$ 
Specific Material $=$

Manufacturer $=$

$\% \mathrm{Al}_{2} \mathrm{O}_{3}=$

$\% \mathrm{Na}_{2} \mathrm{O}=$

Other Formula $=\mathrm{ZnO}$

Other Formula $=$

Young's Modulus $=8.75 E 4$

1st Technique $=$ Controlled Flaw

2nd Technique $=$

$n=$

Environment $=\mathrm{N}_{2}(1)$ at $-196 \mathrm{C}$

Reference $=$ SHINKAI,N.,Thesis by author at Penn. State Univ., 1979

Comments $=$

Specific Material =

Manufacturer =

$\% \mathrm{Al}_{2} \mathrm{O}_{3}=$

$\% \mathrm{~B}_{2} \mathrm{O}_{3}=60$

$\% \mathrm{Na}_{2} \mathrm{O}=$

$\% \mathrm{~K}_{2} \mathrm{O}=$

Other Formula $=\mathrm{ZnO}$

Other Formula =

Other $\%=20$

Other $\%=$
$\% \mathrm{CaO}=$

$\% \mathrm{PbO}=20$

$K_{\mathrm{IC}}=.96$

$K_{\mathrm{IC}}=$
$\% \mathrm{MgO}=$

$\% \mathrm{SiO}_{2}=$

Young's Modulus $=8.75 E 4$

1st Technique $=$ Controlled Flaw

2nd Technique $=$

$K_{\mathrm{IC}}=.79$

$\% \mathrm{CaO}=$

$\% \mathrm{PbO}=20$

Gamma $=5.26$

Gamma $=$

$n=$

Environment $=$ air

Reference $=$ SHINKAI,N.,Thesis by author at Penn. State Univ., 1979

Comments $=$

Specific Material $=$

Manufacturer $=$

$\% \mathrm{Al}_{2} \mathrm{O}_{3}=$

$\% \mathrm{Na}_{2} \mathrm{O}=$

Other Formula $=\mathrm{ZnO}$

Other Formula $=$

Young's Modulus

1st Technique $=$ Controlled Flaw

2nd Technique $=$

$n=$

Environment $=\mathrm{N}_{2}(\mathrm{l})$ at $-196 \mathrm{C}$

Reference $=$ SHINKAI,N.,Thesis by author at Penn. State Univ., 1979

Comments $=$

Specific Material =

Mant. acturer =

$\% \mathrm{Al}_{2} \mathrm{O}_{3}=$

$\% \mathrm{~B}_{2} \mathrm{O}_{3}=60$

$\% \mathrm{Na}_{2} \mathrm{O}=$

$\% \mathrm{~K}_{2} \mathrm{O}=$

Other Formula $=\mathrm{ZnO}$

Other Formula $=$

Other $\%=30$

$K_{\mathrm{IC}}=1.0$

$K_{\mathrm{IC}}=$

$\% \mathrm{CaO}=$

$\% \mathrm{PbO}=10$

$\% \mathrm{MgO}=$

$\% \mathrm{SiO}_{2}=$

Other $\%=30$

Other $\%=$

Other $\%=$

$\% \mathrm{CaO}=$

$\% \mathrm{PbO}=10$

$\% \mathrm{MgO}=$

$\% \mathrm{SiO}_{2}=$

Gamma $=3.55$

Gamma $=$

$\% \mathrm{MgO}=$

$\% \mathrm{SiO}_{2}=$

Young's Modulus $=8.91 E 4$

1st Technique $=$ Controlled Flaw

2nd Technique $=$

$K_{\mathrm{IC}}=.81$

$n=$

Environment $=$ air

Reference = SHINKAI,N., Thesis by author at Penn. State Univ., 1979

Comments $=$ 
Specific Material $=$

Manufacturer $=$

$\% \mathrm{Al}_{2} \mathrm{O}_{3}=$

$\% \mathrm{Na}_{2} \mathrm{O}=$

Other Formula =

Other Formula =

Young's Modulus $=5.47 \mathrm{E} 4$

1st Technique $=$ Controlled Flaw

2nd Technique $=$

$n=$

Environment $=\mathrm{N}_{2}(1)$ at $-196 \mathrm{C}$

Reference $=$ SHINKAI,N., Thesis by author at Penn. State Univ., 1979

Comments $=$

Specific Material =

Manufacturer $=$

$\% \mathrm{Al}_{2} \mathrm{O}_{3}=$

$\% \mathrm{Na}_{2} \mathrm{O}=$

Other Formula $=$

Other Formula =

Young's Modulus $=5.47 \mathrm{E} 4$

lst Technique $=$ Controlled Flaw

2nd Technique $=$

$n=$

Environment $=$ air

Reference $=$ SHINKAI,N.,Thesis by author at Penn. State Univ., 1979

Comments $=$

Specific Material $=$

Manufacturer $=$

$\% \mathrm{Al}_{2} \mathrm{O}_{3}=$

$\% \mathrm{~B}_{2} \mathrm{O}_{3}=70$

$\% \mathrm{Na}_{2} \mathrm{O}=$

$\% \mathrm{~K}_{2} \mathrm{O}=$

Other Formula $=\mathrm{ZnO}$

Other $\%=7.5$

Other Formula =

Other $\%=$

$K_{\mathrm{IC}}=.63$

$K_{\mathrm{IC}}=$

$\% \mathrm{CaO}=$

$\% \mathrm{PbO}=30$

$\% \mathrm{MgO}=$

$\% \mathrm{SiO}_{2}=$

Other $\%=$

$K_{\mathrm{IC}}=1.36$

$K_{\mathrm{IC}}=$

Gamma $=16.91$

Gamma =

Young's Modulus $=6.4 E 4$

1st Technique $=$ Controlled Flaw

2nd Technique $=$

$n=$

Environment $=\mathrm{N}_{2}(1)$ at $-196 \mathrm{C}$

Reference $=$ SHINKAI,N., Thesis by author at Penn. State Univ., 1979

Comments $=$

Specific Material $=$

Manufacturer $=$

\% $\mathrm{Al}_{2} \mathrm{O}_{3}=$

$\% \mathrm{Na}_{2} \mathrm{O}=$

$\% \mathrm{~B}_{2} \mathrm{O}_{3}=70$

$\% \mathrm{~K}_{2} \mathrm{O}=$

Other Formula $=\mathrm{ZnO}$

Other Formula =

Young's Modulus

1st Technique $=$ Controlled Flaw

2nd Technique $=$

Other $\%=7.5$

$\% \mathrm{CaO}=$

$\% \mathrm{PbO}=22.5$

Other $\%=$
$K_{\mathrm{IC}}=.77$

$K_{\text {IC }}=$
$\% \mathrm{PbO}=22.5 \quad \% \mathrm{SiO}_{2}=$

Gamma $=11.82$

Gamma =

$n=$

$\% \mathrm{MgO}=$

$\% \mathrm{SiO}_{2}=$

Environment $=$ air

Reference $=$ SHINKAI,N.,Thesis by author at Penn. State Univ., 1979

Comments $=$ 
Specific Material =

Manufacturer $=$

$\% \mathrm{Al}_{2} \mathrm{O}_{3}=$

$\% \mathrm{~B}_{2} \mathrm{O}_{3}=70$

$\% \mathrm{CaO}=$

$\% \mathrm{MgO}=$

$\% \mathrm{Na}_{2} \mathrm{O}=$

$\% \mathrm{~K}_{2} \mathrm{O}=$

$\% \mathrm{PbO}=15$

$\% \mathrm{SiO}_{2}=$

Other Formula $=\mathrm{ZnO}$

Other $\%=15$

Other Formula =

Other $\%=$

Young's Modulus $=6.97 E 4$

1st Technique $=$ Controlled Flaw

$K_{\mathrm{IC}}=1.24$

2nd Technique $=$

Gamma $=11.03$

$n=$

Environment $=\mathrm{N}_{2}(1)$ at $-196 \mathrm{C}$

Reference $=$ SHINKAI,N.,Thesis by author at Penn. State Univ., 1979

Comments $=$

Specific Material $=$

Manufacturer $=$

$\% \mathrm{Al}_{2} \mathrm{O}_{3}=$

$\% \mathrm{~B}_{2} \mathrm{O}_{3}=70$

$\% \mathrm{Na}_{2} \mathrm{O}=$

$\% \mathrm{~K}_{2} \mathrm{O}=$

$\% \mathrm{CaO}=$

$\% \mathrm{PbO}=15$

Gamma $=$

Other Formula $=\mathrm{ZnO}$

Other $\%=15$

Other Formula =

Other $\%=$

Young's Modulus $=6.97 E 4$

1st Technique $=$ Controlled Flaw

$K_{\text {IC }}=.85$

2nd Technique $=$

$K_{\mathrm{IC}}=$

Gamma $=5.20$

$n=$

Environment $=$ air

Reference $=$ SHINKAI,N.,Thesis by author at Penn. State Univ., 1979

Comments $=$

\section{SIM81}

Specific Material $=$

Manufacturer $=$

$\% \mathrm{Al}_{2} \mathrm{O}_{3}=$

$\% \mathrm{Na}_{2} \mathrm{O}=33$

Other Formula $=$

Other Formula $=$

Young's Modulus =

$$
\begin{array}{ll}
\% \mathrm{~B}_{2} \mathrm{O}_{3}= \\
\% \mathrm{~K}_{2} \mathrm{O}= \\
& \text { Other \%= } \\
& \text { Other \% = }
\end{array}
$$$$
\% \mathrm{MgO}=
$$$$
\% \mathrm{SiO}_{2}=
$$

1st Technique $=\mathrm{DCB}$

Gamma =

2nd Technique $=$

$n=21$

Environment $=$ D.I. $\mathrm{H}_{2} \mathrm{O}$

Reference $=$ SIMMONS,C.J., FREIMAN,S.W., JACTAW, 64, 683-86, 1981

Comments $=$

Specific Material $=$

Manufacturer $=$

\% $\mathrm{Al}_{2} \mathrm{O}_{3}=$

$\% \mathrm{Na}_{2} \mathrm{O}=33$

Other Formula =

Other Formula =

Young's Modulus =

1st Technique $=\mathrm{DCB}$

2nd Technique $=$

$n=21$

Environment $=1 \mathrm{M}$ Cs in D.I. $\mathrm{H}_{2} \mathrm{O}$

Reference $=$ SIMMONS,C.J., FREIMAN,S.W., JACTAW, 64, 683-86, 1981

Comments $=$

$$
\begin{aligned}
& \% \mathrm{~B}_{2} \mathrm{O}_{3}= \\
& \% \mathrm{~K}_{2} \mathrm{O}= \\
& \text { Other \% }= \\
& \text { Other \% = }
\end{aligned}
$$$$
\% \mathrm{CaO}=
$$$$
\% \mathrm{PbO}=
$$

$K_{\mathrm{IC}}=$

$K_{\mathrm{IC}}=$

Gamma $=$

Gamma $=$$$
\% \mathrm{CaO}=
$$$$
\% \mathrm{PbO}=
$$$$
\% \mathrm{MgO}=
$$

$\% \mathrm{MgO}=$

$\% \mathrm{SiO}_{2}=67$

$K_{\mathrm{IC}}=$

$K_{\mathrm{IC}}=$

Gamma =

Gamma = 
Specific Material $=$

Manufacturer =

$\% \mathrm{Al}_{2} \mathrm{O}_{3}=$

$\% \mathrm{~B}_{2} \mathrm{O}_{3}=$

$\% \mathrm{CaO}=$

$\% \mathrm{MgO}=$

$\% \mathrm{Na}_{2} \mathrm{O}=33$

$\% \mathrm{~K}_{2} \mathrm{O}=$

$\% \mathrm{PbO}=$

$\% \mathrm{SiO}_{2}=67$

Other Formula =

Other Formula =

Other \% =

Other $\%=$

Young's Modulus =

1st Technique $=\mathrm{DCB}$

$K_{\mathrm{IC}}=$

Gamma $=$

2nd Technique $=$

Gamma $=$

$n=30$

Environment $=1 \mathrm{M} \mathrm{Li}$ in D.I. $\mathrm{H}_{2} \mathrm{O}$

Reference = SIMMONS,C.J., FREIMAN,S.W., JACTAW, 64, 683-86, 1981

Comments $=$

Specific Material =

Manufacturer $=$

$\% \mathrm{Al}_{2} \mathrm{O}_{3}=$

$\% \mathrm{Na}_{2} \mathrm{O}=33$

Other Formula $=$

Other Formula =

Young's Modulus =

$$
\begin{aligned}
& \% \mathrm{~B}_{2} \mathrm{O}_{3}= \\
& \% \mathrm{~K}_{2} \mathrm{O}= \\
& \\
& \text { Other \%= } \\
& \text { Other \%= }
\end{aligned}
$$

$\% \mathrm{CaO}=$

$\% \mathrm{PbO}=$

$\% \mathrm{MgO}=$

$\% \mathrm{SiO}_{2}=67$

1st Technique $=\mathrm{DCB}$

2nd Technique $=$

$n=93$

$K_{\mathrm{IC}}=$

$\mathrm{Gamma}=$

Gamma $=$

Environment $=$ air, $30 \% \mathrm{RH}$

Reference = SIMMONS,C.J., FREIMAN,S.W., JACTAW, 64, 683-86, 1981

Comments $=$

Specific Material $=$

Manufacturer =

$\% \mathrm{Al}_{2} \mathrm{O}_{3}=$

$\% \mathrm{Na}_{2} \mathrm{O}=25$

Other Formula =

Other Formula =

Young's Modulus =

1st Technique $=\mathrm{DCB}$

$\% \mathrm{~B}_{2} \mathrm{O}_{3}=$

$\% \mathrm{~K}_{2} \mathrm{O}=$

$\mathrm{CaO}=$

$\% \mathrm{MgO}=$

Other $\%=$

$\% \mathrm{PbO}=$

$\% \mathrm{SiO}_{2}=75$

2nd Technique $=$

Other $\%=$

$n=19$

Environment $=$ D.I. $\mathrm{H}_{2} \mathrm{O}$

Reference = SIMMONS,C.J., FREIMAN,S.W., JACTAW, 64, 683-86, 1981

Comments $=$

Specific Material $=$

Manufacturer $=$

$\% \mathrm{Al}_{2} \mathrm{O}_{3}=$

$\% \mathrm{Na}_{2} \mathrm{O}=25$

Other Formula =

Other Formula =

Young's Modulus =

$K_{\mathrm{IC}}=$

$K_{\mathrm{IC}}=$

Gamma $=$

Gamma $=$

1st Technique $=$ DCB

$\% \mathrm{~B}_{2} \mathrm{O}_{3}=$

$\% \mathrm{~K}_{2} \mathrm{O}=$

Other $\%=$

Other $\%=$
$\% \mathrm{CaO}=$

$\% \mathrm{PbO}=$

$K_{\mathrm{IC}}=$

$K_{\mathrm{IC}}=$

Gamma $=$

Gamma $=$

$n=19$

Environment $=1 \mathrm{M}$ Cs in D.I. $\mathrm{H}_{2} \mathrm{O}$

Reference = SIMMONS,C.J., FREIMAN,S.W., JACTAW, 64, 683-86, 1981

Comments $=$ 
Specific Material $=$

Manufacturer $=$

$\% \mathrm{Al}_{2} \mathrm{O}_{3}=$

$\% \mathrm{Na}_{2} \mathrm{O}=25$

$\% \mathrm{~B}_{2} \mathrm{O}_{3}=$

$\% \mathrm{CaO}=$

$\% \mathrm{MgO}=$

Other Formula =

$\% \mathrm{~K}_{2} \mathrm{O}=$

$\% \mathrm{PbO}=$

$\% \mathrm{SiO}_{2}=75$

Other Formula =

Other $\%=$

Other $\%=$

Young's Modulus =

1st Technique $=\mathrm{DCB}$

Gamma $=$

2nd Technique $=$

Gamma $=$

$n=27$

Environment $=1 \mathrm{M} \mathrm{Li}$ in D.I. $\mathrm{H}_{2} \mathrm{O}$

Reference $=$ SIMMONS,C.J., FREIMAN,S.W., JACTAW, 64, 683-86, 1981

Comments $=$

Specific Material $=$

Manufacturer $=$

$\% \mathrm{Al}_{2} \mathrm{O}_{3}=$

$\% \mathrm{Na}_{2} \mathrm{O}=25$

Other Formula =

Other Formula =

Young's Modulus =

1st Technique $=\mathrm{DCB}$

2nd Technique $=$

$n=27$

$\% \mathrm{~B}_{2} \mathrm{O}_{3}=$

$\% \mathrm{~K}_{2} \mathrm{O}=$

Other $\%=$

Other $\%=$
$\% \mathrm{CaO}=$

$\% \mathrm{PbO}=$

$K_{\mathrm{IC}}=$

$K_{\mathrm{IC}}=$
$\% \mathrm{MgO}=$

$\% \mathrm{SiO}_{2}=75$

Environment $=$ air, $30 \% \mathrm{RH}$

Reference $=$ SIMMONS,C.J., FREIMAN,S.W., JACTAW, 64, 683-86, 1981

Comments $=$

Specific Material =

Manufacturer $=$

$\% \mathrm{Al}_{2} \mathrm{O}_{3}=1.75$

$\% \mathrm{Na}_{2} \mathrm{O}=13.4$

Other Formula =

Other Formula =

Young's Modulus =

1st Technique $=\mathrm{DCB}$

$\% \mathrm{~B}_{2} \mathrm{O}_{3}=$

$\% \mathrm{~K}_{2} \mathrm{O}=$

Other $\%=$

Other $\%=$
$\% \mathrm{CaO}=7.44$

$\% \mathrm{PbO}=$

$\% \mathrm{MgO}=5.91$

$\% \mathrm{SiO}_{2}=71.4$

2nd Technique $=$

$n=18.1$

Environment $=\mathrm{H}_{2} \mathrm{O}$

Reference = SIMMONS,C.J., FREIMAN,S.W., JACTAW, 64, 683-86, 1981

Comments $=$

Specific Material =

Manufacturer $=$

$\% \mathrm{Al}_{2} \mathrm{O}_{3}=$

$\% \mathrm{Na}_{2} \mathrm{O}=7$

Other Formula =

Other Formula =

Young's Modulus $=$

1st Technique $=$ DCB

2nd Technique $=$

$n=28.3$

Environment $=\mathrm{H}_{2} \mathrm{O}$

Reference $=$ SIMMONS,C.J., FREIMAN,S.W., JACTAW, 64, 683-86, 1981

Comments $=$
$\% \mathrm{~B}_{2} \mathrm{O}_{3}=23$

$\% \mathrm{~K}_{2} \mathrm{O}=$

Other $\%=$

Other $\%=$
$K_{\mathrm{IC}}=$

$K_{\mathrm{IC}}=$

Gamma $=$

Gamma $=$

Gamma $=$

Gamma = 
Specific Material =

Manufacturer =

$\% \mathrm{Al}_{2} \mathrm{O}_{3}=$

$\% \mathrm{Na}_{2} \mathrm{O}=7$
$\% \mathrm{~B}_{2} \mathrm{O}_{3}=23$

$\% \mathrm{~K}_{2} \mathrm{O}=$

Other $\%=$

Other $\%=$
$\% \mathrm{CaO}=$

$\% \mathrm{PbO}=$

$\% \mathrm{MgO}=$

$\% \mathrm{SiO}_{2}=70$

Other Formula =

Other Formula =

Young's Modulus =

1st Technique $=\mathrm{DCB}$

2nd Technique $=$

$n=31.1$

Environment $=$ air, $50 \% \mathrm{RH}$

Reference = SIMMONS,C.J., FREIMAN,S.W., JACTAW, 64, 683-86, 1981

Comments

\section{SME83}

Specific Material =

Manufacturer =

$\% \mathrm{Al}_{2} \mathrm{O}_{3}=$

$\% \mathrm{Na}_{2} \mathrm{O}=$

$\% \mathrm{~B}_{2} \mathrm{O}_{3}=$

$\% \mathrm{~K}_{2} \mathrm{O}=$

Other Formula $=\mathrm{GeO}_{2}$

Other $\%=100$

$K_{\mathrm{IC}}=$

$K_{\text {IC }}=$

Gamma $=$

Gamma =

$\% \mathrm{CaO}=$

$\% \mathrm{PbO}=$

$\% \mathrm{MgO}=$

$\% \mathrm{SiO}_{2}=$
Other Formula =

Young's Modulus $=4.65 E 4$

1st Technique $=\mathrm{NB}$

Other $\%=$

2nd Technique $=$

$n=$

Environment $=\mathrm{N}_{2}(\mathrm{~g})$

Reference $=$ SMETS,B.M.J., Private Communications, 1983

Comments $=$ Precracked samples, crosshead speed $4.310-6 \mathrm{~m} / \mathrm{s}$, dew point $50 \mathrm{C}$

Specific Material $=$

Manufacturer =

$\% \mathrm{Al}_{2} \mathrm{O}_{3}=$

$\% \mathrm{Na}_{2} \mathrm{O}=2.5$

Other Formula $=\mathrm{GeO}_{2}$

Other Formula =

Young's Modulus $=5.258 E 4$

1st Technique $=$ NB

2nd Technique $=$

$n=$

Environment $=\mathrm{N}_{2}(\mathrm{~g})$

Reference $=$ SMETS,B.M.J., Private Communications, 1983

Comments $=$ Precracked samples, crosshead speed $4.310-6 \mathrm{~m} / \mathrm{s}$, dew point $50 \mathrm{C}$

Specific Material =

Manufacturer $=$

$\% \mathrm{~B}_{2} \mathrm{O}_{3}=$

$\% \mathrm{~K}_{2} \mathrm{O}=$

Other $\%=97.5$

$K_{\text {IC }}=.61$

$K_{\text {IC }}=$

Other $\%=$

$$
K_{\text {IC }}=.69
$$$$
K_{\text {IC }}=
$$

$\% \mathrm{CaO}=$

$\% \mathrm{PbO}=$

$\% \mathrm{MgO}=$

$\% \mathrm{SiO}_{2}=$

Gamma $=3.8$

Gamma $=$

Gamma $=4.3$

Gamma $=$
$\% \mathrm{Al}_{2} \mathrm{O}_{3}=$

$\% \mathrm{Na}_{2} \mathrm{O}=5$

Other Formula $=\mathrm{GeO}_{2}$

$\% \mathrm{~B}_{2} \mathrm{O}_{3}=$

$\% \mathrm{~K}_{2} \mathrm{O}=$

Other $\%=95$

Other Formula $=$

Young's Modulus $=5.84 E 4$

1st Technique $=\mathrm{NB}$

2nd Technique $=$

$n=$

Environment $=\mathrm{N}_{2}(\mathrm{~g})$

Reference $=$ SMETS,B.M.J., Private Communications, 1983

Comments $=$ Precracked samples, crosshead speed $4.310-6 \mathrm{~m} / \mathrm{s}$, dew point $50 \mathrm{C}$
$\% \mathrm{CaO}=$

$\% \mathrm{PbO}=$

$K_{\mathrm{IC}}=.83$

$K_{\mathrm{IC}}=$ 
Specific Material =

Manufacturer $=$

$\% \mathrm{Al}_{2} \mathrm{O}_{3}=$

$\% \mathrm{Na}_{2} \mathrm{O}=7.5$

$\% \mathrm{~B}_{2} \mathrm{O}_{3}=$

$\% \mathrm{~K}_{2} \mathrm{O}=$

Other $\%=92.5$

Other $\%=$

Other Formula $=$

Young's Modulus $=6.196 E 4$

1st Technique $=\mathrm{NB}$

2nd Technique $=$

$K_{\mathrm{IC}}=.82$

$\% \mathrm{CaO}=$

$\% \mathrm{PbO}=$

$\% \mathrm{MgO}=$

$\% \mathrm{SiO}_{2}=$

$n=$

$K_{\text {IC }}=$

Gamma $=5.1$

Gamma $=$

Environment $=\mathrm{N}_{2}(\mathrm{~g})$

Reference $=$ SMETS,B.M.J., Private Communications, 1983

Comments $=$ Precracked samples, crosshead speed $4.310-6 \mathrm{~m} / \mathrm{s}$, dew point $50 \mathrm{C}$

Specific Material $=$

Manufacturer =

$\% \mathrm{Al}_{2} \mathrm{O}_{3}=$

$\% \mathrm{Na}_{2} \mathrm{O}=10$

$\% \mathrm{~B}_{2} \mathrm{O}_{3}=$

$\% \mathrm{~K}_{2} \mathrm{O}=$

Other $\%=90$

Other Formula $=\mathrm{GeO}_{2}$

Other Formula =

Other $\%=$
$\% \mathrm{CaO}=$

$\% \mathrm{PbO}=$
$\% \mathrm{MgO}=$

$\% \mathrm{SiO}_{2}=$

Young's Modulus $=6.12 E 4$

1st Technique $=\mathrm{NB}$

2nd Technique $=$

$K_{\mathrm{IC}}=.93$

$K_{\mathrm{IC}}=$

$n=$

Environment $=\mathrm{N}_{2}(\mathrm{~g})$

Reference $=$ SMETS,B.M.J., Private Communications, 1983

Comments $=$ Precracked samples, crosshead speed $4.310-6 \mathrm{~m} / \mathrm{s}$, dew point $50 \mathrm{C}$

Specific Material $=$

Manufacturer $=$

$\% \mathrm{Al}_{2} \mathrm{O}_{3}=$

$\% \mathrm{Na}_{2} \mathrm{O}=9.9$

$\% \mathrm{~B}_{2} \mathrm{O}_{3}=$

$\% \mathrm{~K}_{2} \mathrm{O}=$

Other Formula $=\mathrm{GeO}_{2}$

Other $\%=90.1$

Other Formula =

Other $\%=$

Young's Modulus $=6.903 E 4$

1 st Technique $=\mathrm{NB}$

2nd Technique $=$

$K_{\mathrm{IC}}=.84$

$K_{\mathrm{IC}}=$

$n=$

Environment $=\mathrm{N}_{2}(\mathrm{~g})$

Reference = SMETS,B.M.J., Private Communications, 1983

Comments $=$ Precracked samples, crosshead speed $4.310-6 \mathrm{~m} / \mathrm{s}$, dew point $50 \mathrm{C}$

Specific Material =

Manufacturer =

$\% \mathrm{Al}_{2} \mathrm{O}_{3}=$

$\% \mathrm{Na}_{2} \mathrm{O}=12.5$

$\% \mathrm{~B}_{2} \mathrm{O}_{3}=$

$\% \mathrm{~K}_{2} \mathrm{O}=$

Other Formula $=\mathrm{GeO}_{2}$

Other $\%=87.5$

Other Formula =

Other $\%=$

$\% \mathrm{CaO}=$

$\% \mathrm{PbO}=$
$\% \mathrm{CaO}=$

$\% \mathrm{PbO}=$

$\% \mathrm{MgO}=$

$\% \mathrm{SiO}_{2}=$

Young's Modulus $=6.752 E 4$

1st Technique $=\mathrm{NB}$

2nd Technique $=$

$K_{\mathrm{IC}}=.87$

Gamma $=6.6$

Gamma $=$

$n=$

Environment $=\mathrm{N}_{2}(\mathrm{~g})$

Reference $=$ SMETS,B.M.J., Private Communications, 1983

Comments $=$ Precracked samples, crosshead speed $4.310-6 \mathrm{~m} / \mathrm{s}$, dew point $50 \mathrm{C}$ 
Specific Material $=$

Manufacturer $=$

$\% \mathrm{Al}_{2} \mathrm{O}_{3}=$

$\% \mathrm{Na}_{2} \mathrm{O}=14$

$\% \mathrm{~B}_{2} \mathrm{O}_{3}=$

$\% \mathrm{~K}_{2} \mathrm{O}=$

Other Formula $=\mathrm{GeO}_{2}$

Other Formula =

Young's Modulus $=7.275 E 4$

1st Technique $=\mathrm{NB}$

2nd Technique $=$

$n=$

Environment $=\mathrm{N}_{2}(\mathrm{~g})$

Reference $=$ SMETS,B.M.J., Private Communications, 1983

Comments $=$ Precracked samples, crosshead speed $4.310-6 \mathrm{~m} / \mathrm{s}$, dew point $50 \mathrm{C}$

Specific Material =

Manufacturer $=$

$\% \mathrm{Al}_{2} \mathrm{O}_{3}=$

$\% \mathrm{Na}_{2} \mathrm{O}=$

$\% \mathrm{~B}_{2} \mathrm{O}_{3}=$

$\% \mathrm{~K}_{2} \mathrm{O}=2.5$

Other Formula $=\mathrm{GeO}_{2}$

Other $\%=97.5$

Other Formula =

Young's Modulus $=5.238 E 4$

1 st Technique $=\mathrm{NB}$

2nd Technique $=$

Other $\%=$
$\% \mathrm{CaO}=$

$\% \mathrm{PbO}=$

$K_{\mathrm{IC}}=.78$

$K_{\mathrm{IC}}=$
$K_{\mathrm{IC}}=.71$

$K_{\mathrm{IC}}=$
$\% \mathrm{MgO}=$

$\% \mathrm{SiO}_{2}=$

$n=$

Environment $=\mathrm{N}_{2}(\mathrm{~g})$

Reference $=$ SMETS,B.M.J., Private Communications, 1983

Comments $=$ Precracked samples, crosshead speed $4.310-6 \mathrm{~m} / \mathrm{s}$, dew point $50 \mathrm{C}$

Specific Material $=$

Manufacturer $=$
$\% \mathrm{Al}_{2} \mathrm{O}_{3}=$
$\% \mathrm{~B}_{2} \mathrm{O}_{3}=$
$\% \mathrm{Na}_{2} \mathrm{O}=$
$\% \mathrm{~K}_{2} \mathrm{O}=5$
$\% \mathrm{CaO}=$
$\% \mathrm{PbO}=$
$\% \mathrm{MgO}=$
$\% \mathrm{SiO}_{2}=$
Other Formula $=\mathrm{GeO}_{2} \quad$ Other $\%=95$
Other Formula =
Other $\%=$
Young's Modulus $=5.593 E 4$
1st Technique $=\mathrm{NB}$
2nd Technique $=$
$K_{\mathrm{IC}}=.86$
$K_{\mathrm{IC}}=$
Gamma $=6.2$
Gamma $=$

Gamma $=4.6$

Gamma $=$

Gamma $=3.9$

Gamma $=$

$\% \mathrm{MgO}=$

$\% \mathrm{SiO}_{2}=$

$n=$

Environment $=\mathrm{N}_{2}(\mathrm{~g})$

Reference $=$ SMETS,B.M.J., Private Communications, 1983

Comments $=$ Precracked samples, crosshead speed $4.310-6 \mathrm{~m} / \mathrm{s}$, dew point $50 \mathrm{C}$

Specific Material =

Manufacturer =

$\% \mathrm{Al}_{2} \mathrm{O}_{3}=\quad \% \mathrm{~B}_{2} \mathrm{O}_{3}=$

$\% \mathrm{Na}_{2} \mathrm{O}=$

$\% \mathrm{~K}_{2} \mathrm{O}=7.5$

Other Formula $=\mathrm{GeO}_{2}$

Other $\%=92.5$

Other Formula =

Other $\%=$

Young's Modulus $=5.8 E 4$

1st Technique $=\mathrm{NB}$

2nd Technique $=$

$K_{\mathrm{IC}}=.79$

$\% \mathrm{CaO}=$

$\% \mathrm{PbO}=$

$\% \mathrm{MgO}=$

$\% \mathrm{SiO}_{2}=$

$n=$

$K_{\mathrm{IC}}=$

Gamma $=5.1$

Gamma $=$

Environment $=\mathrm{N}_{2}(\mathrm{~g})$

Reference $=$ SMETS,B.M.J., Private Communications, 1983

Comments $=$ Precracked samples, crosshead speed $4.310-6 \mathrm{~m} / \mathrm{s}$, dew point $50 \mathrm{C}$ 
Specific Material =

Manufacturer =
$\% \mathrm{Al}_{2} \mathrm{O}_{3}=$
$\% \mathrm{~B}_{2} \mathrm{O}_{3}=$
$\% \mathrm{Na}_{2} \mathrm{O}=$
$\% \mathrm{~K}_{2} \mathrm{O}=10$

$\% \mathrm{CaO}=$

$\% \mathrm{MgO}=$

$\% \mathrm{PbO}=$

$\% \mathrm{SiO}_{2}=$

Other Formula $=\mathrm{GeO}_{2} \quad$ Other $\%=90$

Other Formula $=\quad$ Other $\%=$

Young's Modulus $=6.031 E 4$

1 st Technique $=\mathrm{NB}$

2nd Technique $=$

$K_{\mathrm{IC}}=.75$

$K_{\mathrm{IC}}=$

Gamma $=4.4$

$n=$

Environment $=\mathrm{N}_{2}(\mathrm{~g})$

Reference $=$ SMETS,B.M.J., Private Communications, 1983

Comments $=$ Precracked samples, crosshead speed $4.310-6 \mathrm{~m} / \mathrm{s}$, dew point $50 \mathrm{C}$

Specific Material $=$

Manufacturer $=$
$\% \mathrm{Al}_{2} \mathrm{O}_{3}=$
$\% \mathrm{~B}_{2} \mathrm{O}_{3}=$
$\% \mathrm{Na}_{2} \mathrm{O}=$
$\% \mathrm{~K}_{2} \mathrm{O}=12.5$

$\% \mathrm{CaO}=$

$\% \mathrm{PbO}=$

Gamma $=$

Other Formula $=\mathrm{GeO}_{2}$

Other $\%=87.5$

Other Formula =

Other $\%=$

Young's Modulus $=6.13 E 4$

1st Technique $=\mathrm{NB}$

2nd Technique $=$

$K_{\mathrm{IC}}=.72$

$K_{\mathrm{IC}}=$

Gamma $=3.9$

$n=$

Environment $=\mathrm{N}_{2}(\mathrm{~g})$

Reference $=$ SMETS,B.M.J., Private Communications, 1983

Comments $=$ Precracked samples, crosshead speed $4.310-6 \mathrm{~m} / \mathrm{s}$, dew point $50 \mathrm{C}$

Specific Material $=$

Manufacturer =

$\% \mathrm{Al}_{2} \mathrm{O}_{3}=$

$\% \mathrm{Na}_{2} \mathrm{O}=$

$\% \mathrm{~B}_{2} \mathrm{O}_{3}=$

$\% \mathrm{~K}_{2} \mathrm{O}=15$

Other Formula $=\mathrm{GeO}_{2}$

Other Formula $=$

Young's Modulus =

1st Technique $=\mathrm{NB}$

2nd Technique $=$

$n=$

Environment $=\mathrm{N}_{2}(\mathrm{~g})$

Reference $=$ SMETS,B.M.J., Private Communications, 1983

Comments $=$ Precracked samples, crosshead speed $4.310-6 \mathrm{~m} / \mathrm{s}$, dew point $50 \mathrm{C}$

Specific Material $=$

Manufacturer $=$

$\% \mathrm{Al}_{2} \mathrm{O}_{3}=$

$\% \mathrm{~B}_{2} \mathrm{O}_{3}=$

$\% \mathrm{Na}_{2} \mathrm{O}=$

$\% \mathrm{~K}_{2} \mathrm{O}=16.5$

Other Formula $=\mathrm{GeO}_{2}$

Other Formula =

Young's Modulus $=6.157$ E4

1st Technique $=\mathrm{NB}$

2nd Technique $=$

$n=$

Environment $=\mathrm{N}_{2}(\mathrm{~g})$

Reference $=$ SMETS,B.M.J., Private Communications, 1983

Comments $=$ Precracked samples, crosshead speed $4.310-6 \mathrm{~m} / \mathrm{s}$, dew point $50 \mathrm{C}$
$\% \mathrm{CaO}=$

$\% \mathrm{PbO}=$

$K_{\mathrm{IC}}=.67$

$K_{\mathrm{IC}}=$
\% $\mathrm{CaO}=$

$\% \mathrm{PbO}=$

$K_{\mathrm{IC}}=.62$

$K_{\mathrm{IC}}=$
Other $\%=$
$\% \mathrm{MgO}=$

$\% \mathrm{SiO}_{2}=$
$\% \mathrm{MgO}=$

$\% \mathrm{SiO}_{2}=$

Gamma $=3.4$

$\mathrm{Gamma}=$

$\% \mathrm{MgO}=$

$\% \mathrm{SiO}_{2}=$ 
Specific Material $=$

Manufacturer $=$
$\% \mathrm{Al}_{2} \mathrm{O}_{3}=$
$\% \mathrm{~B}_{2} \mathrm{O}_{3}=$
$\% \mathrm{Na}_{2} \mathrm{O}=$
$\% \mathrm{~K}_{2} \mathrm{O}=17.5$
$\% \mathrm{CaO}=$
$\% \mathrm{MgO}=$
Other Formula $=\mathrm{GeO}_{2}$
Other $\%=82.5$
$\% \mathrm{PbO}=$
$\% \mathrm{SiO}_{2}=$
Other Formula =
Other $\%=$
Young's Modulus $=6.054 E 4$
1st Technique $=\mathrm{NB}$
$K_{\text {IC }}=.62$
2nd Technique $=$
Gamma $=3.0$
Gamma $=$

$n=$

Environment $=\mathrm{N}_{2}(\mathrm{~g})$

Reference $=$ SMETS,B.M.J., Private Communications, 1983

Comments $=$ Precracked samples, crosshead speed $4.310-6 \mathrm{~m} / \mathrm{s}$, dew point $50 \mathrm{C}$

Specific Material =

Manufacturer =

$\% \mathrm{Al}_{2} \mathrm{O}_{3}=$

$\% \mathrm{Na}_{2} \mathrm{O}=$

$\% \mathrm{~B}_{2} \mathrm{O}_{3}=$

$\% \mathrm{~K}_{2} \mathrm{O}=20$

Other $\%=80$

Other Formula $=\mathrm{GeO}_{2}$

Other Formula =

Young's Modulus $=5.781 E 4$

1 st Technique $=\mathrm{NB}$

2nd Technique $=$ Other $\%=$

$n=$

Environment $=\mathrm{N}_{2}(\mathrm{~g})$

Reference $=$ SMETS,B.M.J., Private Communications, 1983

Comments $=$ Precracked samples, crosshead speed $4.310-6 \mathrm{~m} / \mathrm{s}$, dew point $50 \mathrm{C}$

Specific Material =

Manufacturer =

$\% \mathrm{Al}_{2} \mathrm{O}_{3}=$

$\% \mathrm{~B}_{2} \mathrm{O}_{3}=$

$\% \mathrm{~K}_{2} \mathrm{O}=$

$\% \mathrm{CaO}=$

$\% \mathrm{PbO}=$

$\% \mathrm{Na}_{2} \mathrm{O}=$

Other $\%=95$

$K_{\mathrm{IC}}=.65$

$K_{\text {IC }}=$

$\mathrm{Gamma}=3.4$

Gamma $=$

Other Formula $=\mathrm{GeO}_{2}$

Other $\%=$

Other Formula =

Young's Modulus $=4.866 E 4$

1st Technique $=\mathrm{NB}$

2nd Technique $=$

$K_{\mathrm{IC}}=.6$

$\% \mathrm{CaO}=$

$\% \mathrm{PbO}=5$

$\% \mathrm{MgO}=$

$\% \mathrm{SiO}_{2}=$

$n=$

Environment $=\mathrm{N}_{2}(\mathrm{~g})$

Reference $=$ SMETS,B.M.J., Private Communications, 1983

Comments $=$ Precracked samples, crosshead speed $4.310-6 \mathrm{~m} / \mathrm{s}$, dew point $50 \mathrm{C}$

Specific Material =

Manufacturer $=$

$\% \mathrm{Al}_{2} \mathrm{O}_{3}=\quad \% \mathrm{~B}_{2} \mathrm{O}_{3}=$

$\% \mathrm{~K}_{2} \mathrm{O}=$

$K_{\mathrm{IC}}=$

$\mathrm{Gamma}=3.5$

Gamma $=$

$\% \mathrm{MgO}=$

$\% \mathrm{SiO}_{2}=$

$\% \mathrm{Na}_{2} \mathrm{O}=$

$\begin{array}{ll}\text { Other Formula }=\mathrm{GeO}_{2} & \text { Other } \%=85 \\ \text { Other Formula }= & \text { Other } \%=\end{array}$

Young's Modulus $=5.414 E 4$

1st Technique $=\mathrm{NB}$

2nd Technique $=$

$K_{\mathrm{IC}}=.6$

$\% \mathrm{CaO}=$

$\% \mathrm{PbO}=15$

$\% \mathrm{MgO}=$

$\% \mathrm{SiO}_{2}=$

$n=$

Environment $=\mathrm{N}_{2}(\mathrm{~g})$

Reference $=$ SMETS,B.M.J., Private Communications, 1983

Comments $=$ Precracked samples, crosshead speed $4.310-6 \mathrm{~m} / \mathrm{s}$, dew point $50 \mathrm{C}$ 
Specific Material =

Manufacturer $=$

$\% \mathrm{Al}_{2} \mathrm{O}_{3}=$

$\tau_{c} \mathrm{Na}_{2} \mathrm{O}=20.8$

$\% \mathrm{~B}_{2} \mathrm{O}_{3}=4.2$

$\% \mathrm{~K}_{2} \mathrm{O}=$

Other \% =

Other $\%=$
$\% \mathrm{CaO}=$

$\% \mathrm{PbO}=$

$K_{\mathrm{IC}}=.84$

$K_{\mathrm{IC}}=$

Gamma $=5.19$

Gamma $=$
$\% \mathrm{MgO}=$

$\% \mathrm{SiO}_{2}=75$

$n=$

Environment $=$ air

Reference $=$ SWEARENGEN,J.,Fracture Mechanics of Cer.,Vol 4,973-87,1978

Comments $=$

Specific Material $=$

Manufacturer =

$\% \mathrm{Al}_{2} \mathrm{O}_{3}=$

$\% \mathrm{Na}_{2} \mathrm{O}=15$

Other Formula $=$

Other Formula $=$

Young's Modulus $=7.735 E 4$

1st Technique $=\mathrm{NB}$

2nd Technique $=$

$\% \mathrm{~B}_{2} \mathrm{O}_{3}=10$

$\% \mathrm{~K}_{2} \mathrm{O}=$

Other $\%=$

Other $\%=$
$\% \mathrm{CaO}=$

$\% \mathrm{PbO}=$

$K_{\mathrm{IC}}=.88$

$K_{\text {IC }}=$

$n=$

Environment $=$ air

Reference $=$ SWEARENGEN,J.,Fracture Mechanics of Cer.,Vol 4,973-87,1978

Comments $=$

Specific Material $=$

Manufacturer =

$\% \mathrm{Al}_{2} \mathrm{O}_{3}=$

$\% \mathrm{~B}_{2} \mathrm{O}_{3}=14.2$

$\% \mathrm{~K}_{2} \mathrm{O}=$

$\% \mathrm{Na}_{2} \mathrm{O}=10.8$

Other Formula =

Other Formula =

Young's Modulus

1st Technique $=\mathrm{NB}$

2nd Technique $=$

$n=$

Environment $=$ air

Reference = SWEARENGEN,J.,Fracture Mechanics of Cer.,Vol 4,973-87,1978

Commenis $=$

Specific Material $=$

Manufacturer $=$

$\% \mathrm{Al}_{2} \mathrm{O}_{3}=$

$\% \mathrm{Na}_{2} \mathrm{O}=6.2$

Other Formula $=$

Other Formula =
Other $\%=$

Other $\%=$
$\% \mathrm{CaO}=$

$\% \mathrm{PbO}=$

$K_{\mathrm{IC}}=.83$

$K_{\mathrm{IC}}=$
$\% \mathrm{~B}_{2} \mathrm{O}_{3}=18.8$

$\% \mathrm{~K}_{2} \mathrm{O}=$

Other $\%=$

Other $\%=$

Gamma $=5.01$

Gamma $=$

$\% \mathrm{MgO}=$

$\% \mathrm{SiO}_{2}=75$

Young's Modulus $=6.89 E 4$

1st Technique $=\mathrm{NB}$

2nd Technique $=$

$n=$

Environment $=$ air

$\% \mathrm{CaO}=$

$\% \mathrm{PbO}=$

$K_{\mathrm{IC}}=.83$

$K_{\mathrm{IC}}=$
$\% \mathrm{MgO}=$

$\% \mathrm{SiO}_{2}=75$

Reference = SWEARENGEN,J.,Fracture Mechanics of Cer.,Vol 4,973-87,1978

Comments $=$ 
Specific Material =

Manufacturer =

$\% \mathrm{Al}_{2} \mathrm{O}_{3}=$

\% $\mathrm{Na}_{2} \mathrm{O}=$

Other Formula =

Other Formula =

Young's Modulus =

$$
\% \mathrm{~B}_{2} \mathrm{O}_{3}=
$$$$
\% \mathrm{~K}_{2} \mathrm{O}=
$$$$
\text { Other } \%=
$$$$
\text { Other } \%=
$$

$$
\begin{aligned}
& \% \mathrm{CaO}= \\
& \% \mathrm{PbO}=
\end{aligned}
$$

$\% \mathrm{MgO}=$

$\% \mathrm{SiO}_{2}=100$

$$
\begin{aligned}
& K_{\text {IC }}= \\
& K_{\text {IC }}=
\end{aligned}
$$

2nd Technique $=$

$n=29.2$

Environment $=$ air, $2 \% \mathrm{RH}$

Reference $=$ TARIYAL,B.K., KALISH,D., Frac. Mech. of Cer. 3,161-75,1978

Comments $=$ Surface condition-plastic-clad fiber (EVA-single coating)

Specific Material $=$

Manufacturer $=$

$\% \mathrm{Al}_{2} \mathrm{O}_{3}=$

$\% \mathrm{Na}_{2} \mathrm{O}=$

Other Formula $=$

Other Formula =

Young's Modulus =

$\% \mathrm{~B}_{2} \mathrm{O}_{3}=$

$\% \mathrm{~K}_{2} \mathrm{O}=$

Other $\%=$

Other $\%=$

$\% \mathrm{CaO}=$

$\% \mathrm{PbO}=$

$K_{\text {IC }}=$

$K_{\text {IC }}=$

2nd Technique $=$

$n=21.8$

Environment $=$ air, $45 \% \mathrm{RH}$

Reference = TARIYAL,B.K., KALISH,D., Frac. Mech. of Cer. 3,161-75,1978

Comments $=$ Surface condition-plastic-clad fiber (EVA-single coating)

Specific Material $=$

Manufacturer =

$\% \mathrm{Al}_{2} \mathrm{O}_{3}=$

$\% \mathrm{Na}_{2} \mathrm{O}=$

Other Formula $=$

Other Formula =

Young's Modulus =

1st Technique $=$ Dynamic Fat.

2nd Technique $=$

$n=16.2$

Environment $=$ air, $71 \% \mathrm{RH}$

Reference = TARIYAL,B.K., KALISH,D., Frac. Mech. of Cer. 3,161-75, 1978

Comments $=$ Surface condition-plastic-clad fiber (EVA-single coating)

Specific Material $=$

Manufacturer =

$\% \mathrm{Al}_{2} \mathrm{O}_{3}=$

$\% \mathrm{Na}_{2} \mathrm{O}=$

Other Formula =

Other Formula =

Young's Modulus =
$\% \mathrm{~B}_{2} \mathrm{O}_{3}=$

$\% \mathrm{~K}_{2} \mathrm{O}=$

Other $\%=$

Other $\%=$
$\% \mathrm{CaO}=$

$\% \mathrm{PbO}=$

$K_{\text {IC }}=$

$K_{\mathrm{IC}}=$

1st Technique $=$ Dynamic Fat.

2nd Technique $=$

$n=15.3$

Environment $=$ air, $97 \% \mathrm{RH}$

Reference = TARIYAL,B.K., KALISH,D., Frac. Mech. of Cer. 3,161-75,1978

Comments $=$ Surface condition-plastic-clad fiber (EVA-single coating)

$$
\begin{array}{ll}
\% \mathrm{CaO}= & \% \mathrm{MgO}= \\
\% \mathrm{PbO}= & \% \mathrm{SiO}_{2}=100
\end{array}
$$

$K_{\mathrm{IC}}=$

$K_{\mathrm{IC}}=$

Gamma $=$

Gamma $=$ 
Specific Material =

Manufacturer $=$

$\% \mathrm{Al}_{2} \mathrm{O}_{3}=$

$\% \mathrm{Na}_{2} \mathrm{O}=$

Other Formula =

Other Formula =

Young's Modulus =
$\% \mathrm{~B}_{2} \mathrm{O}_{3}=$

$\% \mathrm{~K}_{2} \mathrm{O}=$

Other $\%=$

Other $\%=$
$\% \mathrm{CaO}=$

$\% \mathrm{PbO}=$
$\% \mathrm{MgO}=$

$\% \mathrm{SiO}_{2}=100$ 1st Technique $=$ Dynamic Fat.

2nd Technique $=$

$K_{\text {IC }}=$

$K_{\text {IC }}=$

Gamma $=$

Gamma $=$

Environment $=$ air, $45 \% \mathrm{RH}$

Reference $=$ TARIYAL,B.K., KALISH,D., Frac. Mech. of Cer. 3,161-75,1978,

Comments $=$ Surface cond.-plastic-clad fiber(silane and EVA-dual coating)

Specific Material $=$

Manufacturer $=$

$\% \mathrm{Al}_{2} \mathrm{O}_{3}=$

$\% \mathrm{Na}_{2} \mathrm{O}=$

Other Formula $=$

Other Formula =

Young's Modulus =

1st Technique $=$ Dynamic Fat.

2nd Technique $=$

$n=16.8$

Environment $=$ air, $97 \% \mathrm{RH}$

Reference $=$ TARIYAL,B.K., KALISH,D., Frac. Mech. of Cer. 3,161-75,1978

Comments $=$ Surface cond.-plastic-clad fiber(silane and EVA-dual coating)

Specific Material $=$

Manufacturer =

$\% \mathrm{Al}_{2} \mathrm{O}_{3}=$

$\% \mathrm{Na}_{2} \mathrm{O}=$

Other Formula =

Other Formula =

Young's Modulus =

1st Technique $=$ Dynamic Fat.

2nd Technique $=$

$n=27.6$

Environment $=$ air, $45 \%$ RH

Reference $=$ TARIYAL,B.K., KALISH,D., Frac. Mech. of Cer. 3,161-75,1978

Comments $=$ Surface condition-plastic-clad fiber (single coating)

Specific Material =

Manufacturer $=$

$\% \mathrm{Al}_{2} \mathrm{O}_{3}=$

$\% \mathrm{Na}_{2} \mathrm{O}=$

$\% \mathrm{~B}_{2} \mathrm{O}_{3}=$

$\% \mathrm{~K}_{2} \mathrm{O}=$

Other $\%=$

Other $\%=$

$K_{\mathrm{IC}}=$

$\% \mathrm{CaO}=$

$\% \mathrm{PbO}=$

$\% \mathrm{~B}_{2} \mathrm{O}_{3}=$

$\% \mathrm{~K}_{2} \mathrm{O}=$

$\% \mathrm{CaO}=$

$\% \mathrm{PbO}=$
$K_{\mathrm{IC}}=$

$K_{\mathrm{IC}}=$

$\% \mathrm{CaO}=$

$\% \mathrm{PbO}=$

$\% \mathrm{MgO}=$

$\% \mathrm{SiO}_{2}=100$

Gamma $=$

Gamma =

Other Formula =

Other Formula =

$\% \mathrm{MgO}=$

$\% \mathrm{SiO}_{2}=100$

Young's Modulus =

1st Technique $=$ Dynamic Fat.

$\% \mathrm{MgO}=$

Other \% =

$\% \mathrm{SiO}_{2}=100$

2nd Technique $=$

Other $\%=$

Gamma =

Gamma =

$n=25.3$

Environment $=$ air, $97 \% \mathrm{RH}$

Reference $=$ TARIYAL,B.K., KALISH,D., Frac. Mech. of Cer. 3,161-75, 1978

Comments $=$

$K_{\mathrm{IC}}=$

$K_{\mathrm{IC}}=$

Gamma $=$

Gamma $=$ 
Specific Material $=$

Manufacturer $=$

$\% \mathrm{Al}_{2} \mathrm{O}_{3}=$
$\% \mathrm{~B}_{2} \mathrm{O}_{3}=$

$\% \mathrm{~K}_{2} \mathrm{O}=$

Other $\%=$

Other $\%=$
$\% \mathrm{CaO}=$

$\% \mathrm{PbO}=$

$K_{\text {IC }}=$

$K_{\text {IC }}=$

2nd Technique $=$

$n=14.7$

Environment $=$ air, $97 \% \mathrm{RH}$

Reference = TARIYAL,B.K., KALISH,D., Frac. Mech. of Cer. 3,161-75,1978

Comments $=$ Surface condition-plastic-clad fiber

\section{VER80}

Specific Material =

Manufacturer $=$

$\begin{array}{ll}\% \mathrm{Al}_{2} \mathrm{O}_{3}= & \% \mathrm{~B}_{2} \mathrm{O}_{3}=100 \\ \% \mathrm{Na}_{2} \mathrm{O}= & \% \mathrm{~K}_{2} \mathrm{O}= \\ \text { Other Formula }= & \text { Other } \%= \\ \text { Other Formula }= & \text { Other } \%=\end{array}$

$\% \mathrm{CaO}=$

$\% \mathrm{PbO}=$

Other $\%=$
$\% \mathrm{MgO}=$

$\% \mathrm{SiO}_{2}=100$

Gamma $=$

Gamma $=$

Young's Modulus $=5.7 \mathrm{E} 4$

lst Technique $=\mathrm{NB}$

$K_{\mathrm{IC}}=1.45$

2nd Technique $=$

Gamma $=18.44$

Gamma $=$

$n=$

Environment $=$ air

Reference = VERNAZ,E., Journal of Non-Crystal. Solids 37, 359-65,1980

Comments $=$ Young's Modulus is extrapolated

Specific Material =

Manufacturer $=$

$\% \mathrm{Al}_{2} \mathrm{O}_{3}=$

$\% \mathrm{Na}_{2} \mathrm{O}=1$

Other Formula =

Other Formula =

Young's Modulus $=5.6 E 4$

1st Technique $=\mathrm{NB}$

2nd Technique $=$
$\% \mathrm{~B}_{2} \mathrm{O}_{3}=99$
$\% \mathrm{~K}_{2} \mathrm{O}=$
Other $\%=$
Other $\%=$

$\% \mathrm{CaO}=$

$\% \mathrm{MgO}=$

$\% \mathrm{PbO}=$

$\% \mathrm{MgO}=$

$\% \mathrm{SiO}_{2}=$

$n=$

Environment $=$ air

Reference = VERNAZ,E., Journal of Non-Crystal. Solids 37, 359-65,1980

Comments $=$ Young's Modulus is extrapolated

Specific Material =

Manufacturer $=$

$\% \mathrm{Al}_{2} \mathrm{O}_{3}=$

$\% \mathrm{Na}_{2} \mathrm{O}=2$

Other Formula $=$

Other Formula =

Young's Modulus $=4.9 E 4$

1st Technique $=$ NB

$K_{\text {IC }}=1.4$

Gamma $=17.5$

$K_{\text {IC }}=$

Gamma $=$

$\% \mathrm{SiO}_{2}=$

2nd Technique $=$

$K_{\text {IC }}=1.25$

$\% \mathrm{CaO}=$

$\% \mathrm{MgO}=$

$\% \mathrm{~B}_{2} \mathrm{O}_{3}=98$

$\% \mathrm{PbO}=$

$\% \mathrm{SiO}_{2}=$

$n=$

Environment $=$ air

Other $\%=$

Other $\%=$

$K_{\text {IC }}=$

Gamma $=15.94$

Gamma $=$

Reference = VERNAZ,E., Journal of Non-Crystal. Solids 37, 359-65,1980

Comments $=$ Young's Modulus is extrapolated 
Specific Material = Manufacturer =
$\% \mathrm{Al}_{2} \mathrm{O}_{3}=$
$\% \mathrm{~B}_{2} \mathrm{O}_{3}=95$
$\% \mathrm{Na}_{2} \mathrm{O}=5$
$\% \mathrm{~K}_{2} \mathrm{O}=$
Other Formula =
Other $\%=$
Other Formula =
Other $\%=$

$\% \mathrm{CaO}=$

$\% \mathrm{MgO}=$

$\% \mathrm{PbO}=$

$\% \mathrm{SiO}_{2}=$

Young's Modulus $=4.8 E 4$

1st Technique $=\mathrm{NB}$

$K_{\mathrm{IC}}=1.2$

$K_{\text {IC }}=$

Gamma $=15$

2nd Technique $=$

$n=$

Environment $=$ air

Reference $=$ VERNAZ,E., Journal of Non-Crystal. Solids 37, 359-65,1980

Comments $=$ Young's Modulus is extrapolated

Specific Material $=$

Manufacturer $=$

$\% \mathrm{Al}_{2} \mathrm{O}_{3}=$

$\% \mathrm{Na}_{2} \mathrm{O}=10$

Other Formula $=$

Other Formula $=$

Young's Modulus $=4.2 E 4$

1st Technique $=\mathrm{NB}$

$\% \quad \mathrm{~B}_{2} \mathrm{O}_{3}=90$

$\% \mathrm{~K}_{2} \mathrm{O}=$

Other $\%=$

Other $\%=$

Gamma =

2nd Technique $=$

$K_{\mathrm{IC}}=1.05$

$\% \mathrm{CaO}=$

$\% \mathrm{MgO}=$

$\% \mathrm{PbO}=$

$\% \mathrm{SiO}_{2}=$

$n=$

$K_{\mathrm{IC}}=$

Gamma $=13.13$

Gamma $=$

Environment $=$ air

Reference = VERNAZ,E., Journal of Non-Crystal. Solids 37, 359-65,1980

Comments $=$ Young's Modulus is extrapolated

Specific Material $=$

Manufacturer $=$

$\% \mathrm{Al}_{2} \mathrm{O}_{3}=$

$\% \mathrm{Na}_{2} \mathrm{O}=20$

$\% \mathrm{~B}_{2} \mathrm{O}_{3}=80$

$\% \mathrm{~K}_{2} \mathrm{O}=$

$\% \mathrm{CaO}=$

$\% \mathrm{PbO}=$

$\% \mathrm{MgO}=$

Other $\%=$

Other Formula $=$

Other $\%=$

$\% \mathrm{SiO}_{2}=$

Young's Modulus $=3.6 E 4$

1st Technique $=\mathrm{NB}$

2nd Technique $=$

$K_{\mathrm{IC}}=.95$

$K_{\mathrm{IC}}=$

$\mathrm{Gamma}=12.54$

$n=$

Environment $=$ air

Reference = VERNAZ,E., Journal of Non-Crystal. Solids 37, 359-65,1980

Comments $=$ Young's Modulus is extrapolated

Specific Material =

Manufacturer $=$

$\% \mathrm{Al}_{2} \mathrm{O}_{3}=$

$\% \mathrm{Na}_{2} \mathrm{O}=34$

Other Formula =

$\% \mathrm{~B}_{2} \mathrm{O}_{3}=66$

$\% \mathrm{~K}_{2} \mathrm{O}=$

$\% \mathrm{CaO}=$

$\% \mathrm{MgO}=$

Other $\%=$

$\% \mathrm{PbO}=$

$\% \mathrm{SiO}_{2}=$

Other Formula =

Other $\%=$

Gamma $=$

Young's Modulus $=2.8 E 4$

1st Technique $=\mathrm{NB}$

$K_{\text {IC }}=.65$

$K_{\mathrm{IC}}=$

Gamma $=7.55$

2nd Technique $=$

Gamma $=$

$n=$

Environment $=$ air

Reference $=$ VERNAZ,E., Journal of Non-Crystal. Solids 37, 359-65,1980

Comments $=$ Young's Modulus is extrapolated 
Specific Material =

Manufacturer =
$\% \mathrm{Al}_{2} \mathrm{O}_{3}=$
$\% \mathrm{~B}_{2} \mathrm{O}_{3}=90$
$\% \mathrm{CaO}=$
$\% \mathrm{MgO}=$
$\% \mathrm{Na}_{2} \mathrm{O}=10$
$\% \mathrm{~K}_{2} \mathrm{O}=$
$\% \mathrm{PbO}=$
$\% \mathrm{SiO}_{2}=$
Other Formula =
Other $\%=$
Other Formula =
Other $\%=$
Young's Modulus $=3.9 E 4$
1st Technique $=\mathrm{DT}$
$K_{\mathrm{IC}}=1.0$
$\mathrm{Gamma}=12.82$
2nd Technique $=$
$K_{\text {IC }}=$
Gamma $=$

$n=$

Environment $=$ air

Reference = VERNAZ,E., Journal of Non-Crystal. Solids 37, 359-65,1980

Comments $=$ Young's Modulus is extrapolated

Specific Material $=$

Manufacturer $=$

$\begin{array}{ll}\% \mathrm{Al}_{2} \mathrm{O}_{3}= & \% \mathrm{~B}_{2} \mathrm{O}_{3}=80 \\ \% \mathrm{Na}_{2} \mathrm{O}=20 & \% \mathrm{~K}_{2} \mathrm{O}= \\ \text { Other Formula }= & \text { Other \%= } \\ \text { Other Formula }= & \quad \text { Other } \%=\end{array}$

$\% \mathrm{CaO}=\quad \% \mathrm{MgO}=$

$\% \mathrm{PbO}=\quad \% \mathrm{SiO}_{2}=$

Young's Modulus $=3.3 \mathrm{E} 4$

1 st Technique $=\mathrm{DT}$

$K_{\mathrm{IC}}=.85$

2nd Technique $=$

$K_{1 \mathrm{C}}=$

Gamma $=10.95$

$n=$

Environment $=$ air

Reference = VERNAZ,E., Journal of Non-Crystal. Solids 37, 359-65,1980

Comments $=$ Young's Modulus is extrapolated

Specific Material $=$

Manufacturer =

$\% \mathrm{Al}_{2} \mathrm{O}_{3}=$

$\% \mathrm{Na}_{2} \mathrm{O}=25$

$\% \mathrm{~B}_{2} \mathrm{O}_{3}=75$

$\% \mathrm{~K}_{2} \mathrm{O}=$

$\% \mathrm{CaO}=$

$\% \mathrm{PbO}=$

Gamma $=$

Other Formula $=$

Other $\%=$

Other Formula =

Other $\%=$

Young's Modulus $=3.0 \mathrm{E} 4$

1st Technique $=\mathrm{DT}$

2nd Technique $=$

$n=$

Environment $=$ air

Reference = VERNAZ,E., Journal of Non-Crystal. Solids 37, 359-65,1980

Comments $=$ Young's Modulus is extrapolated

Specific Material =

Manufacturer =

$\% \mathrm{Al}_{2} \mathrm{O}_{3}=$

$\% \mathrm{Na}_{2} \mathrm{O}=34$

Other Formula =

$\% \mathrm{~B}_{2} \mathrm{O}_{3}=66$

$\% \mathrm{~K}_{2} \mathrm{O}=$

Other $\%=$

$K_{\mathrm{IC}}=.77$

$K_{\mathrm{IC}}=$

Other $\%=$
$\% \mathrm{CaO}=$

$\% \mathrm{PbO}=$

$K_{\text {IC }}=.7$

$K_{\text {IC }}=$

Gamma $=9.88$

Gamma $=$

Young's Modulus $=2.6 E 4$

1 st Technique $=\mathrm{DT}$

2nd Technique $=$

$n=$

Environment $=$ air

Reference = VERNAZ,E., Journal of Non-Crystal. Solids 37, 359-65,1980

Comments $=$ Young's Modulus is extrapolated 
Specific Material =

Manufacturer $=$

$\% \mathrm{Al}_{2} \mathrm{O}_{3}=$

$\% \mathrm{Na}_{2} \mathrm{O}=35$

Other Formula =

Other Formula =

Young's Modulus $=2.2 E 4$

1st Technique $=\mathrm{DT}$

2nd Technique $=$

$\% \mathrm{~B}_{2} \mathrm{O}_{3}=65$

$\% \mathrm{~K}_{2} \mathrm{O}=$

$\% \mathrm{CaO}=$

$\% \mathrm{MgO}=$

Other \% =

$\% \mathrm{PbO}=$

$\% \mathrm{SiO}_{2}=$

$n=$

Environment $=$ air

Reference $=$ VERNAZ,E., Journal of Non-Crystal. Solids 37, 359-65,1980

Comments $=$ Young's Modulus is extrapolated

Specific Material $=$

Manufacturer $=$

$\% \mathrm{Al}_{2} \mathrm{O}_{3}=$

$\% \mathrm{Na}_{2} \mathrm{O}=7.5$

Other Formula =

Other Formula =

Young's Modulus $=6.225 E 4$

1st Technique $=$ NB

2nd Technique $=$

Gamma $=7.38$

$K_{\mathrm{IC}}=$

Gamma $=$

$n=$

Environment $=$ air

Reference $=$ VERNAZ,E., Journal of Non-Crystal. Solids 37, 359-65,1980

Comments $=$ Young's Modulus is extrapolated

Specific Material $=$

Manufacturer $=$

$\% \mathrm{Al}_{2} \mathrm{O}_{3}=$

$\% \mathrm{Na}_{2} \mathrm{O}=12.5$

$\% \mathrm{~B}_{2} \mathrm{O}_{3}=$

$\% \mathrm{CaO}=$

$\% \mathrm{PbO}=$

$\% \mathrm{MgO}=$

Other $\%=$

Other $\%=$

$\% \mathrm{SiO}_{2}=92.5$

Other Formula =

Other Formula =

Young's Modulus $=6.2 \mathrm{E} 4$

1st Technique $=\mathrm{NB}$

$K_{\mathrm{IC}}=1.1$

$K_{\text {IC }}=$

Gamma $=9.72$

2nd Technique $=$

$K_{\text {IC }}=1.09$

$\% \mathrm{CaO}=$

$\% \mathrm{MgO}=$

$\% \mathrm{~K}_{2} \mathrm{O}=$

$\% \mathrm{PbO}=$

Gamma $=$

Other \% =

Other $\%=$

$\% \mathrm{SiO}_{2}=87.5$

$n=$

Environment $=$ air

Reference $=$ VERNAZ,E., Journal of Non-Crystal. Solids 37, 359-65,1980

Comments $=$ Young's Modulus is extrapolated

Specific Material =

Manufacturer $=$

$\% \mathrm{Al}_{2} \mathrm{O}_{3}=\quad \% \mathrm{~B}_{2} \mathrm{O}_{3}=$

$\% \mathrm{Na}_{2} \mathrm{O}=20$

Other Formula =

$\% \mathrm{~K}_{2} \mathrm{O}=$

$\% \mathrm{CaO}=$

$\% \mathrm{MgO}=$

Other $\%=$

Other Formula $=$

Other $\%=$

$\% \mathrm{PbO}=$

Gamma $=9.58$

Gamma $=$

Young's Modulus $=5.7 E 4$

1st Technique $=\mathrm{NB}$

2nd Technique $=$

$K_{\mathrm{IC}}=.87$

$K_{\mathrm{IC}}=$

Gamma $=6.64$

$n=$

Environment $=$ air

Reference $=$ VERNAZ,E., Journal of Non-Crystal. Solids 37, 359-65, 1980

Comments $=$ Young's Modulus is extrapolated 
Specific Material =

Manufacturer =

$\% \mathrm{Al}_{2} \mathrm{O}_{3}=$

$\% \mathrm{Na}_{2} \mathrm{O}=30$

Other Formula $=$

Other Formula $=$

Young's Modulus $=5.8 E 4$

1st Technique $=\mathrm{NB}$

2nd Technique $=$

$n=$

Environment $=$ air

Reference = VERNAZ,E., Journal of Non-Crystal. Solids 37, 359-65, 1980

Comments $=$ Young's Modulus is extrapolated

Specific Material $=$

Manufacture $=$

$\% \mathrm{Al}_{2} \mathrm{O}_{3}=$

$\% \mathrm{Na}_{2} \mathrm{O}=38$

Other Formula $=$

Other Formula =

Young's Modulus $=6.0 E 4$

1st Technique $=\mathrm{NB}$

2nd Technique $=$

$n=$

Environment $=$ air

Reference = VERNAZ,E., Journal of Non-Crystal. Solids 37, 359-65,1980

Comments $=$ Young's Modulus is extrapolated

Specific Material =

Manufacturer $=$

\% $\mathrm{Al}_{2} \mathrm{O}_{3}=$

$\% \mathrm{Na}_{2} \mathrm{O}=45$

Other Formula $=$

Other Formula $=$

Young's Modulus

$\% \mathrm{~B}_{2} \mathrm{O}_{3}=$

$\% \mathrm{~K}_{2} \mathrm{O}=$

Other $\%=$

Other $\%=$
$\% \mathrm{CaO}=$

$\% \mathrm{PbO}=$

$K_{\text {IC }}=.9$

$K_{\mathrm{IC}}=$

$\% \mathrm{CaO}=$

$\% \mathrm{PbO}=$

$K_{\text {IC }}=1.03$

$K_{\text {IC }}=$

$\% \mathrm{CaO}=$

$\% \mathrm{PbO}=$

$\% \mathrm{~K}_{2} \mathrm{O}=$

Other \% =

Other $\%=$

Gamma $=6.98$

Gamma $=$

$\% \mathrm{MgO}=$

$\% \mathrm{SiO}_{2}=70$

$\% \mathrm{MgO}=$

$\% \mathrm{SiO}_{2}=62$

Gamma $=8.84$

Gamma =

$\% \mathrm{MgO}=$

$\% \mathrm{SiO}_{2}=55$

1st Technique $=\mathrm{NB}$

2nd Technique $=$

$K_{\mathrm{IC}}=.9$

$K_{\mathrm{IC}}=$

Gamma $=7.01$

$n=$

Environment $=$ air

Reference = VERNAZ,E., Journal of Non-Crystal. Solids 37, 359-65,1980

Comments $=$ Young's Modulus is extrapolated

Specific Material =

Manufacturer $=$

$\% \mathrm{Al}_{2} \mathrm{O}_{3}=$

$\% \mathrm{Na}_{2} \mathrm{O}=$

Other Formula $=$

Other Formula =

Young's Modulus $=13.8 E 4$

1st Technique $=\mathrm{NB}$

2nd Technique $=$

$n=$

Environment $=$ air

Reference $=$ VERNAZ,E., Journal of Non-Crystal. Solids 37, 359-65,1980

Comments $=$ Young's Modulus is extrapolated

$$
\begin{array}{ll}
\% \mathrm{CaO}= & \% \mathrm{MgO}^{2} \\
\% \mathrm{PbO}=24 & \% \mathrm{SiO}_{2}=76
\end{array}
$$

Other $\%=$

Other $\%=$

$$
K_{\mathrm{IC}}=1.1
$$$$
K_{\mathrm{IC}}=
$$

Gamma $=4.38$

Gamma $=$ 
Specific Material =

Manufacturer =

$\% \mathrm{Al}_{2} \mathrm{O}_{3}=$

$\% \mathrm{Na}_{2} \mathrm{O}=$

Other Formula =

Other Formula $=$

Young's Modulus $=12.8 E 4$

ist Technique $=\mathrm{NB}$

2nd Technique $=$

$n=$

Environment $=$ air

Reference $=$ VERNAZ,E., Journal of Non-Crystal. Solids 37, 359-65,1980

Comments $=$ Young's Modulus is extrapolated

Specific Material $=$

Manufacturer =

$\% \mathrm{Al}_{2} \mathrm{O}_{3}=$

$\% \mathrm{Na}_{2} \mathrm{O}=$

Other Formula =

Other Formula =

Young's Modulus $=11.7 E 4$

ist Technique $=\mathrm{NB}$

2nd Technique $=$

$n=$

Environment $=$ air

Reference = VERNAZ,E., Journal of Non-Crystal. Solids 37, 359-65,1980

Comments $=$ Young's Modulus is extrapolated

Specific Material $=$

Manufacturer =
$\% \mathrm{Al}_{2} \mathrm{O}_{3}=$

$\% \mathrm{Na}_{2} \mathrm{O}=$

Other Formula $=$

Young's Modulus $=7.7 E 4$

ist Technique $=\mathrm{NB}$

2nd Technique $=$

$n=$

Environment $=$ air

Reference = VERNAZ,E., Journal of Non-Crystal. Solids 37, 359-65,1980

Comments $=$ Young's Modulus is extrapolated

Specific Material $=$

Manufacturer =
$\% \mathrm{Al}_{2} \mathrm{O}_{3}=$
$\% \mathrm{~B}_{2} \mathrm{O}_{3}=82$
$\% \mathrm{Na}_{2} \mathrm{O}=$
Other Formula $=$
Other Formula $=$
$\% \mathrm{~K}_{2} \mathrm{O}=$
Other $\%=$
Other $\%=$

Young's Modulus = $5.1 E 4$

1st Technique $=$ NB

2nd Technique $=$

$n=$

Environment $=$ air

Comments $=$ Young's Modulus is extrapolated
Other Formula $=$

Reference = VERNAZ,E., Journal of Non-Crystal. Solids 37, 359-65, 1980
$K_{\text {IC }}=1.0$

$K_{\text {IC }}=$

Gamma $=3.91$

Gamma =
$K_{\text {IC }}=.9$

$K_{\mathrm{lC}}=$

$\% \mathrm{CaO}=$

$\% \mathrm{PbO}=60$

$K_{\mathrm{IC}}=.5$

$K_{\mathrm{IC}}=$
$\% \mathrm{CaO}=$

$\% \mathrm{PbO}=18$

$K_{\text {IC }}=1.15$

$K_{\mathrm{IC}}=$
Gamma $=3.46$
$\% \mathrm{MgO}=$
Gamma =
$\% \mathrm{MgO}=$

$\% \mathrm{SiO}_{2}=60$
$\% \mathrm{SiO}_{2}=73$

$\% \mathrm{PbO}=27$

$\% \mathrm{PbO}=40$

$$
\% \mathrm{SiO}_{2}=60
$$

Other $\%=$
\% $\mathrm{MgO}=$

$\% \mathrm{SiO}_{2}=40$
Gamma $=1.62$

Gamma = 
Specific Material $=$

Manufacturer $=$

$\% \mathrm{Al}_{2} \mathrm{O}_{3}=$

$\% \mathrm{Na}_{2} \mathrm{O}=$

Other Formula $=$

Other Formula =

Young's Modulus $=4.4 E 4$

1st Technique $=\mathrm{NB}$

2nd Technique $=$

$n=$

Environment $=$ air

Reference = VERNAZ,E., Journal of Non-Crystal. Solids 37, 359-65,1980

Comments $=$ Young's Modulus is extrapolated

Specific Material $=$

Manufacturer $=$

$\% \mathrm{Al}_{2} \mathrm{O}_{3}=$

$\% \mathrm{Na}_{2} \mathrm{O}=$

Other Formula $=$

Other Formula =

Young's Modulus $=3.9 E 4$

1st Technique $=\mathrm{NB}$

2nd Technique $=$

$\% \mathrm{~B}_{2} \mathrm{O}_{3}=72$

$\% \mathrm{~K}_{2} \mathrm{O}=$

Other \% =

$\% \mathrm{CaO}=$

$\% \mathrm{PbO}=28$

$\% \mathrm{MgO}=$

$\% \mathrm{SiO}_{2}=$

Other $\%=$
$K_{\mathrm{IC}}=.85$

$K_{\mathrm{IC}}=$

Gamma $=8.21$

Gamma $=$
$K_{\mathrm{IC}}=.65$

$K_{\mathrm{IC}}=$
$\% \mathrm{CaO}=$

$\% \mathrm{PbO}=34.5$

$\% \mathrm{MgO}=$

$\% \mathrm{SiO}_{2}=$

Other \% =

Other $\%=$ $n=$

Environment $=$ air

Reference = VERNAZ,E., Journal of Non-Crystal. Solids 37, 359-65,1980

Comments $=$ Young's Modulus is extrapolated

Specific Material =

Manufacturer $=$

$\% \mathrm{Al}_{2} \mathrm{O}_{3}=$

$\% \mathrm{Na}_{2} \mathrm{O}=$

Other Formula $=$

Other Formula =

Young's Modulus $=3.15 E 4$

1st Technique $=\mathrm{NB}$

2nd Technique $=$

$n=$

Environment $=$ air

Reference = VERNAZ,E., Journal of Non-Crystal. Solids 37, 359-65,1980

Comments $=$ Young's Modulus is extrapolated

Specific Material =

Manufacturer $=$

\% $\mathrm{Al}_{2} \mathrm{O}_{3}=$

$\% \mathrm{Na}_{2} \mathrm{O}=$

Other Formula $=$

Other Formula $=$

Young's Modulus $=3.7 E 4$

1st Technique $=\mathrm{NB}$

2nd Technique $=$

$\% \mathrm{~B}_{2} \mathrm{O}_{3}=50$

$\% \mathrm{~K}_{2} \mathrm{O}=$

Other \% =

Other $\%=$
Gamma $=5.42$

Gamma $=$
$\% \mathrm{CaO}=$

$\% \mathrm{PbO}=50$

$\% \mathrm{MgO}=$

$\% \mathrm{SiO}_{2}=$

$K_{\mathrm{IC}}=.38$

$K_{\mathrm{IC}}=$

Gamma $=2.29$

Gamma =

$n=$

Environment $=$ air

Reference $=$ VERNAZ,E., Journal of Non-Crystal. Solids 37, 359-65,1980

Comments $=$ Young's Modulus is extrapolated

$$
K_{\mathrm{IC}}=.58
$$$$
K_{\mathrm{IC}}=
$$

$\% \mathrm{CaO}=$

$\% \mathrm{PbO}=60$

$\% \mathrm{MgO}=$

$\% \mathrm{SiO}_{2}=$

Other $\%=$

Other $\%=$
Gamma $=4.55$

Gamma $=$ 
Specific Material =

Manufacturer $=$

$\% \mathrm{Al}_{2} \mathrm{O}_{3}=$

$\% \mathrm{Na}_{2} \mathrm{O}=$

$\% \mathrm{~B}_{2} \mathrm{O}_{3}=50$

$\% \mathrm{~K}_{2} \mathrm{O}=$

Other Formula $=\mathrm{ZnO}$

Other Formula =

Young's Modulus $=4.0 E$

1st Technique $=\mathrm{NB}$

2nd Technique $=$

$n=$

Environment $=$ air

Reference $=$ VERNAZ,E., Journal of Non-Crystal. Solids 37, 359-65,1980

Comments $=$ Young's Modulus is extrapolated

Specific Material =

Manufacturer $=$

$\% \mathrm{Al}_{2} \mathrm{O}_{3}=$

$\% \mathrm{Na}_{2} \mathrm{O}=$

$\% \mathrm{~B}_{2} \mathrm{O}_{3}=45$

$\% \mathrm{~K}_{2} \mathrm{O}=$

Other Formula $=\mathrm{ZnO}$

Other Formula =

Young's Modulus $=4.2 E 4$

1st Technique $=\mathrm{NB}$

2nd Technique $=$

Other $\%=55$

Other $\%=$

$K_{\mathrm{IC}}=.78$
$\% \mathrm{CaO}=$

$\% \mathrm{PbO}=$

$K_{\mathrm{IC}}=.7$

$K_{\mathrm{IC}}=$

Gamma $=6.13$

Gamma =
$\% \mathrm{MgO}=$

$\% \mathrm{SiO}_{2}=$

$n=$

Environment $=$ air

Reference $=$ VERNAZ,E., Journal of Non-Crystal. Solids 37, 359-65,1980

Comments $=$ Young's Modulus is extrapolated

Specific Material =

Manufacturer $=$

$\% \mathrm{Al}_{2} \mathrm{O}_{3}=$

$\% \mathrm{Na}_{2} \mathrm{O}=$

Other Formula $=\mathrm{ZnO}$

Other Formula $=$

Young's Modulus $=4.25 E 4$

1st Technique $=\mathrm{NB}$

2nd Technique $=$

$n=$

Environment $=$ air

Reference = VERNAZ,E., Journal of Non-Crystal. Solids 37, 359-65,1980

Comments $=$ Young's Modulus is extrapolated

Specific Material $=$

Manufacturer $=$

$\% \mathrm{Al}_{2} \mathrm{O}_{3}=$

$\% \mathrm{Na}_{2} \mathrm{O}=$

$\% \mathrm{~B}_{2} \mathrm{O}_{3}=40$

$\% \mathrm{~K}_{2} \mathrm{O}=$

Other $\%=60$

Other $\%=$

$\% \mathrm{~B}_{2} \mathrm{O}_{3}=$

$\% \mathrm{~K}_{2} \mathrm{O}=$

$K_{\text {IC }}=.80$

$\% \mathrm{CaO}=$

$\% \mathrm{PbO}=$

$K_{\mathrm{IC}}=$
$K_{\mathrm{IC}}=$

$\% \mathrm{CaO}=$

$\% \mathrm{PbO}=$

$\% \mathrm{MgO}=$

$\% \mathrm{SiO}_{2}=$

Gamma $=$

Other Formula $=\mathrm{GeO}_{2}$

$\% \mathrm{MgO}=$

$\% \mathrm{SiO}_{2}=$

Gamma $=7.24$

Other Formula =

Young's Modulus $=2.65 E 4$

1st Technique $=\mathrm{NB}$

Other $\%=100$

Gamma $=7.44$

2nd Technique $=$

Other $\%=$

Gamma =

$n=$

Environment $=$ air

Reference = VERNAZ,E., Journal of Non-Crystal. Solids 37, 359-65, 1980

Comments $=$ Young's Modulus is extrapolated

$K_{\text {IC }}=.67$

$K_{\mathrm{IC}}=$

$\% \mathrm{CaO}=$

$\% \mathrm{PbO}=$

$\% \mathrm{MgO}=$

$\% \mathrm{SiO}_{2}=$

Gamma $=8.47$

Gamma $=$ 
Specific Material =

Manufacturer $=$

$\% \mathrm{Al}_{2} \mathrm{O}_{3}=$

$\% \mathrm{~B}_{2} \mathrm{O}_{3}=$

$\% \mathrm{CaO}=$

$\% \mathrm{MgO}=$

$\% \mathrm{Na}_{2} \mathrm{O}=4$

$\% \mathrm{~K}_{2} \mathrm{O}=$

$\% \mathrm{PbO}=$

$\% \mathrm{SiO}_{2}=$

Other Formula $=\mathrm{GeO}_{2} \quad$ Other $\%=96$

Other Formula =

Other $\%=$

Young's Modulus $=4.2 E 4$

1st Technique $=$ NB

2nd Technique $=$

$K_{\mathrm{IC}}=1.06$

Gamma $=13.38$

$n=$

Gamma =

Environment $=$ air

Reference = VERNAZ,E., Journal of Non-Crystal. Solids 37, 359-65,1980

Comments $=$ Young's Modulus is extrapolated

Specific Material $=$

Manufacturer $=$

$\% \mathrm{Al}_{2} \mathrm{O}_{3}=$

$\% \mathrm{~B}_{2} \mathrm{O}_{3}=$

$\% \mathrm{Na}_{2} \mathrm{O}=9$

$\% \mathrm{~K}_{2} \mathrm{O}=$

$\% \mathrm{CaO}=$

$\% \mathrm{PbO}=$

$\% \mathrm{MgO}=$

Other Formula $=\mathrm{GeO}_{2}$

Other $\%=91$

Other Formula =

Other $\%=$

Young's Modulus $=4.9 E 4$

1st Technique $=\mathrm{NB}$

2nd Technique $=$

$K_{\mathrm{IC}}=1.27$

$K_{\mathrm{IC}}=$

Gamma $=16.46$

$n=$

Environment $=$ air

Reference = VERNAZ,E., Journal of Non-Crystal. Solids 37, 359-65,1980

Comments $=$ Young's Modulus is extrapolated

Specific Material =

Manufacturer $=$

$\% \mathrm{Al}_{2} \mathrm{O}_{3}=$

$\% \mathrm{~B}_{2} \mathrm{O}_{3}=$

$\% \mathrm{Na}_{2} \mathrm{O}=10$

$\% \mathrm{~K}_{2} \mathrm{O}=$

Other Formula $=\mathrm{GeO}_{2}$

Other $\%=90$

$\% \mathrm{CaO}=$

$\% \mathrm{PbO}=$

$\% \mathrm{MgO}=$

Other Formula =

Other $\%=$

Young's Modulus $=5.2 \mathrm{E} 4$

1st Technique $=\mathrm{NB}$

2nd Technique $=$

$n=$

Environment $=$ air

Reference = VERNAZ,E., Journal of Non-Crystal. Solids 37, 359-65,1980

Comments $=$ Young's Modulus is extrapolated

Specific Material =

Manufacturer $=$

$\% \mathrm{Al}_{2} \mathrm{O}_{3}=$

$\% \mathrm{~B}_{2} \mathrm{O}_{3}=$

$\% \mathrm{Na}_{2} \mathrm{O}=12$

$\% \mathrm{~K}_{2} \mathrm{O}=$

Other Formula $=\mathrm{GeO}_{2}$

Other $\%=88$

$\% \mathrm{CaO}=$

$\% \mathrm{MgO}=$

Other Formula =

Other $\%=$

$\% \mathrm{PbO}=$

Gamma $=$

Young's Modulus $=4.7 E 4$

1st Technique $=\mathrm{NB}$

2nd Technique $=$

$K_{\text {IC }}=$

Gamma $=15.32$

$n=$

Environment $=$ air

Reference = VERNAZ,E., Journal of Non-Crystal. Solids 37, 359-65,1980

Comments $=$ Young's Modulus is extrapolated 
Specific Material =

Manufacturer $=$

$\% \mathrm{Al}_{2} \mathrm{O}_{3}=\quad \% \mathrm{~B}_{2} \mathrm{O}_{3}=$

$\% \mathrm{Na}_{2} \mathrm{O}=37 \quad \% \mathrm{~K}_{2} \mathrm{O}=$

$\% \mathrm{CaO}=$

$\% \mathrm{PbO}=$

Other $\%=63$

Other $\%=$

Other Formula =

Young's Modulus $=2.45 E 4$

1st Technique $=\mathrm{NB}$

$K_{\mathrm{IC}}=.58$

$K_{\mathrm{IC}}=$

Gamma $=6.87$

2nd Technique $=$

$n=$

Environment $=$ air

Reference $=$ VERNAZ,E., Journal of Non-Crystal. Solids 37, 359-65,1980

Comments $=$ Young's Modulus is extrapolated

Specific Material $=$

Manufacturer $=$

$\% \mathrm{Al}_{2} \mathrm{O}_{3}=$

$\% \mathrm{Na}_{2} \mathrm{O}=20$

$\% \mathrm{~B}_{2} \mathrm{O}_{3}=80$

$\% \mathrm{~K}_{2} \mathrm{O}=$

Other $\%=$

$\% \mathrm{CaO}=$

$\% \mathrm{MgO}=$

Other Formula $=$

Other $\%=$

$\% \mathrm{PbO}=$

$\% \mathrm{SiO}_{2}=$

Other Formula =

Young's Modulus $=6.0 E 4$

1st Technique $=\mathrm{NB}$

2nd Technique $=$

$K_{\mathrm{IC}}=.93$

$K_{\mathrm{IC}}=$

Gamma $=7.21$

$n=$

Environment $=$ air

Reference $=$ VERNAZ,E., Journal of Non-Crystal. Solids 37, 359-65,1980

Comments $=$ Young's Modulus is extrapolated

Specific Material $=$

Manufacturer $=$

$\% \mathrm{Al}_{2} \mathrm{O}_{3}=$

$\% \mathrm{Na}_{2} \mathrm{O}=20$

Other Formula $=$

Other Formula =

Young's Modulus $=6.0 E 4$

1st Technique $=\mathrm{NB}$

$\% \quad \mathrm{~B}_{2} \mathrm{O}_{3}=70$

$\% \mathrm{~K}_{2} \mathrm{O}=$

Other $\%=$

Other $\%=$

Gamma $=$

2nd Technique $=$

$n=$

Environment $=$ air

Reference = VERNAZ,E., Journal of Non-Crystal. Solids 37, 359-65, 1980

Comments $=$ Young's Modulus is extrapolated

Specific Material $=$

Manufacturer $=$

$\% \mathrm{Al}_{2} \mathrm{O}_{3}=$

$\% \mathrm{Na}_{2} \mathrm{O}=20$

Other Formula $=$

Other Formula =

IC $=.84$

$\% \mathrm{CaO}=$

$\% \mathrm{PbO}=$

$\% \mathrm{MgO}=$

$\% \mathrm{SiO}_{2}=10$

Young's Modulus $=6.0 \mathrm{E} 4$

1st Technique $=\mathrm{NB}$

$\% \mathrm{~B}_{2} \mathrm{O}_{3}=60$

$\% \mathrm{~K}_{2} \mathrm{O}=$

Other $\%=$

Other $\%=$

Gamma $=5.88$

Gamma $=$

2nd Technique $=$

$n=$

Environment $=$ air

Reference = VERNAZ,E., Journal of Non-Crystal. Solids 37, 359-65,1980

Comments $=$ Young's Modulus is extrapolated

$K_{\mathrm{IC}}=.83$

$K_{\mathrm{IC}}=$
$\% \mathrm{CaO}=$

$\% \mathrm{PbO}=$

$\% \mathrm{MgO}=$

$\% \mathrm{SiO}_{2}=20$

Gamma $=5.74$

Gamma $=$ 
Specific Material =

Manufacturer $=$

$\% \mathrm{Al}_{2} \mathrm{O}_{3}=$

$\% \mathrm{Na}_{2} \mathrm{O}=20$

Other Formula =

Other Formula =

Young's Modulus $=6.0 \mathrm{E} 4$

1st Technique $=\mathrm{NB}$

2nd Technique $=$

$n=$

Environment $=$ air

Reference = VERNAZ,E., Journal of Non-Crystal. Solids 37, 359-65, 1980

Comments $=$ Young's Modulus is extrapolated

Specific Material $=$

Manufacturer $=$

$\% \mathrm{Al}_{2} \mathrm{O}_{3}=$

$\% \mathrm{Na}_{2} \mathrm{O}=20$

Other Formula =

Other Formula =

Young's Modulus $=6.0 \mathrm{E} 4$

1st Technique $=\mathrm{NB}$

2nd Technique $=$

$n=$

Environment $=$ air

Reference = VERNAZ,E., Journal of Non-Crystal. Solids 37, 359-65, 1980

Comments $=$ Young's Modulus is extrapolated

Specific Material $=$

Manufacturer $=$

$\% \mathrm{Al}_{2} \mathrm{O}_{3}=$

$\% \mathrm{Na}_{2} \mathrm{O}=20$

Other Formula $=$

Other Formula =

Young's Modulus $=6.0 E 4$

1st Technique $=\mathrm{NB}$

2nd Technique $=$

$n=$

Environment $=$ air

Reference $=$ VERNAZ,E., Journal of Non-Crystal. Solids 37, 359-65, 1980

Comments $=$ Young's Modulus is extrapolated

Specific Material $=$

Manufacturer =

$\% \mathrm{Al}_{2} \mathrm{O}_{3}=$

$\% \mathrm{Na}_{2} \mathrm{O}=20$

Other Formula $=$

Other Formula =

Young's Modulus $=6.0 E 4$

1st Technique $=\mathrm{NB}$

2nd Technique $=$

$\% \mathrm{~B}_{2} \mathrm{O}_{3}=30$

$\% \mathrm{~K}_{2} \mathrm{O}=$

Other $\%=$

Other $\%=$

$$
K_{\mathrm{IC}}=1.0
$$$$
K_{\mathrm{IC}}=
$$

$\% \mathrm{CaO}=$

$\% \mathrm{PbO}=$

Gamma $=8.33$
Gamma $=$

$\% \mathrm{MgO}=$

$\% \mathrm{SiO}_{2}=30$

$K_{\mathrm{IC}}=1.0$

$K_{\text {IC }}=$
$\% \mathrm{MgO}=$

$\% \mathrm{SiO}_{2}=40$

Gamma $=8.33$

Gamma $=$
$K_{\mathrm{IC}}=1.1$

$K_{\mathrm{IC}}=$

$\% \mathrm{CaO}=$

$\% \mathrm{PbO}=$

$\% \mathrm{MgO}=$

$\% \mathrm{SiO}_{2}=50$

Gamma $=10.08$

Gamma $=$
$K_{\text {IC }}=1.08$

$K_{\text {IC }}=$

$\% \mathrm{CaO}=$

$\% \mathrm{PbO}=$

Other $\%=$

Other $\%=$
$\% \mathrm{MgO}=$

$\% \mathrm{SiO}_{2}=60$

Gamma $=9.72$

Gamma =

$n=$

Environment $=$ air

Reference = VERNAZ,E., Journal of Non-Crystal. Solids 37, 359-65,1980

Comments $=$ Young's Modulus is extrapolated 
Specific Material =

Manufacturer $=$

$\% \mathrm{Al}_{2} \mathrm{O}_{3}=$

$\% \mathrm{Na}_{2} \mathrm{O}=20$

$\% \mathrm{~B}_{2} \mathrm{O}_{3}=10$

$\% \mathrm{CaO}=$

$\% \mathrm{MgO}=$

Other Formula =

$\% \mathrm{~K}_{2} \mathrm{O}=$

$\% \mathrm{PbO}=$

$\% \mathrm{SiO}_{2}=70$

Other Formula =

Other $\%=$

Other $\%=$

Young's Modulus $=6.0 \mathrm{E} 4$

1st Technique $=\mathrm{NB}$

2nd Technique $=$

$K_{\text {IC }}=.91$

$K_{\text {IC }}=$

Gamma $=6.90$

$n=$

Environment $=$ air

Reference = VERNAZ,E., Journal of Non-Crystal. Solids 37, 359-65,1980

Comments $=$ Young's Modulus is extrapolated

Specific Material =

Manufacturer =

$\% \mathrm{Al}_{2} \mathrm{O}_{3}=$

$\% \mathrm{Na}_{2} \mathrm{O}=20$

Other Formula =

Other Formula =

$\% \mathrm{~B}_{2} \mathrm{O}_{3}=$

$\% \mathrm{~K}_{2} \mathrm{O}=$

Other $\%=$

Other $\%=$

Gamma $=$

Young's Modulus $=6.0 \mathrm{E} 4$

1st Technique $=\mathrm{NB}$

2nd Technique $=$

$K_{\mathrm{IC}}=.86$

$\% \mathrm{CaO}=$

$\% \mathrm{PbO}=$

$\% \mathrm{MgO}=$

$\% \mathrm{SiO}_{2}=80$

$n=$

$K_{\mathrm{IC}}=$

Gamma $=6.16$

Gamma =

Environment $=$ air

Reference $=$ VERNAZ,E., Journal of Non-Crystal. Solids 37, 359-65,1980

Comments $=$ Young's Modulus is extrapolated

\section{WIE69}

Specific Material $=7944$

Manufacturer $=$ Corning Glass

$\% \mathrm{Al}_{2} \mathrm{O}_{3}=\quad \% \mathrm{~B}_{2} \mathrm{O}_{3}=$

$\% \mathrm{Na}_{2} \mathrm{O}=$

$\% \mathrm{~K}_{2} \mathrm{O}=$

Other Formula $=$

Other $\%=$

Other Formula =

Other $\%=$

$\% \mathrm{CaO}=$

$\% \mathrm{PbO}=$

Young's Modulus $=7.21 E 4$

1st Technique $=\mathrm{DCB}$

2nd Technique $=$

$n=$

Environment $=\mathrm{N}_{2}(\mathrm{~g})$

Reference $=$ WIEDERHORN,S., JACTAW, Vol. 52, No. 2, 99-105, 1969

Comments $=$

Specific Material $=7900$

Manufacturer $=$ Corning Glass

$\% \mathrm{Al}_{2} \mathrm{O}_{3}=.594 \quad \% \mathrm{~B}_{2} \mathrm{O}_{3}=2.61$

$\% \mathrm{Na}_{2} \mathrm{O}=$

Other Formula $=$

Other Formula =

Young's Modulus $=6.59 E 4$

1 st Technique $=\mathrm{DCB}$

2nd Technique $=$

$\% \mathrm{~K}_{2} \mathrm{O}=$

Other $\%=$

Other $\%=$

$K_{\mathrm{IC}}=.79$

$K_{\mathrm{IC}}=$

$n=$

Environment $=\mathrm{N}_{2}(\mathrm{~g})$

Reference = WIEDERHORN,S., JACTAW, Vol. 52, No. 2, 99-105, 1969

Comments $=$
$\% \mathrm{CaO}=$

$\% \mathrm{PbO}=$

$K_{\text {IC }}=.72$

$K_{\mathrm{IC}}=$
$\% \mathrm{MgO}=$

$\% \mathrm{SiO}_{2}=99.9$

Gamma $=4.37$

Gamma $=$ 
Specific Material $=1720$

Manufacturer $=$ Corning Glass
$\% \mathrm{Al}_{2} \mathrm{O}_{3}=12.0$
$\% \mathrm{~B}_{2} \mathrm{O}_{3}=3.54$
$\% \mathrm{Na}_{2} \mathrm{O}=.994$
$\% \mathrm{~K}_{2} \mathrm{O}=$
Other Formula $=$
Other $\%=$
Other Formula =
Other $\%=$

$\% \mathrm{CaO}=6.59$

$\% \mathrm{MgO}=18.3$

$\% \mathrm{PbO}=$

$\% \mathrm{SiO}_{2}=58.4$

Young's Modulus $=8.91 E 4$

1st Technique $=\mathrm{DCB}$

$K_{\text {IC }}=.91$

2nd Technique $=$

$K_{\mathrm{IC}}=$

Gamma $=4.66$

$n=$

Environment $=\mathrm{N}_{2}(\mathrm{~g})$

Reference $=$ WIEDERHORN,S., JACTAW, Vol. 52, No. 2, 99-105, 1969

Comments $=$

Specific Material $=7740$

Manufacturer $=$ Corning Glass

$\begin{array}{ll}\% \mathrm{Al}_{2} \mathrm{O}_{3}=1.21 & \% \mathrm{~B}_{2} \mathrm{O}_{3}=12.4 \\ \% \mathrm{Na}_{2} \mathrm{O}=3.99 & \% \mathrm{~K}_{2} \mathrm{O}= \\ \text { Other Formula }= & \text { Other } \%= \\ \text { Other Formula }= & \text { Other } \%=\end{array}$

$\% \mathrm{CaO}=$

$\% \mathrm{PbO}=$

Gamma $=$

$\% \mathrm{MgO}=$

Young's Modulus $=6.37 E 4$

1st Technique $=$ DCB

2nd Technique $=$

$n=$

Environment $=\mathrm{N}_{2}(\mathrm{~g})$

Reference = WIEDERHORN,S., JACTAW, Vol. 52, No. 2, 99-105, 1969

Comments $=$

Specific Material =

Manufacturer $=$ Corning Glass

$\begin{array}{ll}\% \mathrm{Al}_{2} \mathrm{O}_{3}=1.16 & \% \mathrm{~B}_{2} \mathrm{O}_{3}= \\ \% \mathrm{Na}_{2} \mathrm{O}=13.4 & \% \mathrm{~K}_{2} \mathrm{O}=.632 \\ \text { Other Formula }= & \text { Other } \%= \\ \text { Other Formula }= & \text { Other } \%=\end{array}$

$K_{\text {IC }}=.77$

$K_{\text {IC }}=$

Gamma $=4.63$

Gamma $=$

$\% \mathrm{SiO}_{2}=82.3$

Young's Modulus $=7.34 E 4$

1st Technique $=\mathrm{DCB}$

$K_{\mathrm{IC}}=.75$

$\% \mathrm{CaO}=7.43$

$\% \mathrm{MgO}=5.91$

$\% \mathrm{PbO}=$

$\% \mathrm{SiO}_{2}=71.3$

2nd Technique $=$

$K_{\text {IC }}=$

$n=$

Environment $=\mathrm{N}_{2}(\mathrm{~g})$

Reference $=$ WIEDERHORN,S., JACTAW, Vol. 52, No. 2, 99-105, 1969

Comments $=$

Specific Material $=0041$

Manufacturer $=$ Corning Glass

$\% \mathrm{Al}_{2} \mathrm{O}_{3}=2.97 \quad \% \mathrm{~B}_{2} \mathrm{O}_{3}=$

$\% \mathrm{Na}_{2} \mathrm{O}=12.2$

$\% \mathrm{~K}_{2} \mathrm{O}=1.6$

$\% \mathrm{CaO}=$

$\% \mathrm{MgO}=$

Other Formula $=$

Other $\%=$

$\% \mathrm{PbO}=7.59$

Gamma $=3.86$

Other Formula =

Other $\%=$

Gamma $=$

Young's Modulus $=6.53 E 4$

1st Technique $=\mathrm{DCB}$

2nd Technique $=$

$K_{\text {IC }}=.68$

$K_{\mathrm{IC}}=$

Gamma $=3.52$

$n=$

Environment $=\mathrm{N}_{2}(\mathrm{~g})$

Reference $=$ WIEDERHORN,S., JACTAW, Vol. 52, No. 2, 99-105, 1969

Comments $=$ 
Specific Material $=7944$

Manufacturer $=$ Corning Glass
$\% \mathrm{Al}_{2} \mathrm{O}_{3}=$
$\% \mathrm{~B}_{2} \mathrm{O}_{3}=$
$\% \mathrm{CaO}=$
$\% \mathrm{MgO}=$
$\% \mathrm{Na}_{2} \mathrm{O}=$
$\% \mathrm{~K}_{2} \mathrm{O}=$
$\% \mathrm{PbO}=$
Other Formula $=$
Other $\%=$
Other Formula =
Other $\%=$
Young's Modulus =
1st Technique $=\mathrm{DCB}$
$K_{\mathrm{IC}}=$
Gamma $=$
2nd Technique $=$
$K_{\mathrm{IC}}=$
Gamma =

$\% \mathrm{SiO}_{2}=99.9$

$n=40.8$

Environment $=\mathrm{H}_{2} \mathrm{O}$

Reference $=$ WIEDERHORN,S.M.,BOLZ,L.,JACTAW,Vol. 53,No. 10,543-48,1970

Comments $=$

Specific Material $=1720$

Manufacturer $=$ Corning Glass

$\% \mathrm{Al}_{2} \mathrm{O}_{3}=12.0 \quad \% \mathrm{~B}_{2} \mathrm{O}_{3}=3.54$

$\% \mathrm{~K}_{2} \mathrm{O}=$

$\% \mathrm{CaO}=6.59$

$\% \mathrm{MgO}=18.3$

$\% \mathrm{Na}_{2} \mathrm{O}=.994$

Other $\%=$

$\% \mathrm{PbO}=$

$\% \mathrm{SiO}_{2}=58.4$

Other Formula =

Other $\%=$

Young's Modulus =

1st Technique $=\mathrm{DCB}$

2nd Technique $=$

$n=26.5$

Environment $=\mathrm{H}_{2} \mathrm{O}$

Reference = WIEDERHORN,S.M.,BOLZ,L.,JACTAW,Vol. 53,No. 10,543-48,1970

Comments $=$

Specific Material $=$

Manufacturer $=$ Corning Glass

$\% \mathrm{Al}_{2} \mathrm{O}_{3}=10.8 \quad \% \mathrm{~B}_{2} \mathrm{O}_{3}=$

$\% \mathrm{Na}_{2} \mathrm{O}=13.0 \quad \% \mathrm{~K}_{2} \mathrm{O}=2.33$

$K_{\mathrm{IC}}=$

Gamma $=$

$K_{\mathrm{IC}}=$

Gamma $=$

Other Formula $=\mathrm{TiO}_{2}$

Other $\%=.649$

$\% \mathrm{CaO}=.462$

$\% \mathrm{PbO}=$

$\% \mathrm{MgO}=5.79$

Other Formula $=\mathrm{As}_{2} \mathrm{O}_{3}$

Other $\%=<.1 \mathrm{~W} \%$

Young's Modulus =

1st Technique $=\mathrm{DCB}$

2nd Technique $=$

$K_{\mathrm{IC}}=$

Gamma $=$

$n=28.7$

$K_{\mathrm{IC}}=$

Gamma =

Environment $=\mathrm{H}_{2} \mathrm{O}$

Reference $=$ WIEDERHORN,S.M.,BOLZ,L.,JACTAW,Vol. 53,No. 10,543-48, 1970

Comments $=$

Specific Material $=7740$

Manufacturer $=$ Corning Glass

$\% \mathrm{Al}_{2} \mathrm{O}_{3}=1.21$

$\% \mathrm{~B}_{2} \mathrm{O}_{3}=12.4$

$\% \mathrm{CaO}=$

$\mathrm{SiO}_{2}=66.6$

$\% \mathrm{Na}_{2} \mathrm{O}=3.99$

$\% \mathrm{~K}_{2} \mathrm{O}=$

$\% \mathrm{PbO}=$

$\% \mathrm{MgO}=$

Other Formula =

Other \% =

Other Formula =

Other $\%=$

$\% \mathrm{SiO}_{2}=82.3$

Young's Modulus =

lst Technique $=\mathrm{DCB}$

2nd Technique $=$

$K_{\text {IC }}=$

Gamma $=$

$n=35.5$

$K_{\mathrm{IC}}=$

Gamma $=$

Environment $=\mathrm{H}_{2} \mathrm{O}$

Reference = WIEDERHORN,S.M.,BOLZ,L.,JACTAW,Vol. 53,No. 10,543-548, 1970

Comments $=$ 
Specific Material $=0041$

Manufacturer $=$ Corning Glass
$\% \mathrm{Al}_{2} \mathrm{O}_{3}=2.97$
$\% \mathrm{~B}_{2} \mathrm{O}_{3}=$
$\% \mathrm{Na}_{2} \mathrm{O}=12.2$
$\% \mathrm{~K}_{2} \mathrm{O}=1.60$
Other $\%=$
Other $\%=$
Other Formula =

$\% \mathrm{CaO}=$

$\% \mathrm{PbO}=7.59$

$\% \mathrm{MgO}=$

Young's Modulus =

1st Technique $=$ DCB

2nd Technique $=$

$n=25.2$

Environment $=\mathrm{H}_{2} \mathrm{O}$

Reference = WIEDERHORN,S.M.,BOLZ,L.,JACTAW,Vol. 53,No. 10,543-548,1970

Comments $=$

Specific Material =

Manufacturer $=$ Corning Glass
$\% \mathrm{Al}_{2} \mathrm{O}_{3}=1.16$
$\% \mathrm{~B}_{2} \mathrm{O}_{3}=$
$\% \mathrm{Na}_{2} \mathrm{O}=13.4$
$\% \mathrm{~K}_{2} \mathrm{O}=.632$
$\% \mathrm{CaO}=7.43$
$\% \mathrm{PbO}=$
Other Formula =
Other $\%=$
Other Formula =
Young's Modulus =
1st Technique $=$ DCB
Other $\%=$
2nd Technique $=$
$n=18.5$
Environment $=\mathrm{H}_{2} \mathrm{O}$
Reference = WIEDERHORN,S.M.,BOLZ,L.,JACTAW,Vol. 53,No.10,543-548, 1970
Comments $=$$$
K_{\mathrm{IC}}=
$$
$K_{\mathrm{IC}}=$

$\% \mathrm{SiO}_{2}=75.6$

$$
\begin{array}{ll}
K_{\mathrm{IC}}= & \text { Gamma }= \\
K_{\mathrm{IC}}= & \text { Gamma }=
\end{array}
$$

WIE73

Specific Material =

Manufacturer $=$

$\% \mathrm{Al}_{2} \mathrm{O}_{3}=1.16$

$\% \mathrm{Na}_{2} \mathrm{O}=13.4$

Other Formula =

Other Formula =

Young's Modulus =

$$
\text { Gamma }=
$$

Gamma $=$

1st Technique $=$ DCB

2nd Technique $=$

$n=19.4$

$$
\begin{array}{ll}
\% & \mathrm{~B}_{2} \mathrm{O}_{3}= \\
\% & \mathrm{~K}_{2} \mathrm{O}=.632 \\
& \text { Other \% }= \\
& \text { Other \% = }
\end{array}
$$

$\% \mathrm{CaO}=7.43$

$\% \mathrm{PbO}=$

$\% \mathrm{MgO}=5.91$

$\% \mathrm{SiO}_{2}=71.3$

$$
K_{\mathrm{IC}}=
$$$$
K_{\mathrm{IC}}=
$$

Gamma $=$

Gamma $=$

Environment $=6 \mathrm{~N} \mathrm{NaOH}$

Reference = WIEDERHORN,S.M., JOHNSON,H., JACTAW, 56, 192-97, 1973

Comments $=$

Specific Material $=$

Manufacturer $=$

$\% \mathrm{Al}_{2} \mathrm{O}_{3}=1.16$

$\% \mathrm{Na}_{2} \mathrm{O}=13.4$

Other Formula =

Other Formula =

Young's Modulus =

1st Technique $=\mathrm{DCB}$

2nd Technique $=$

$n=32$

$$
\begin{array}{ll}
\% \mathrm{~B}_{2} \mathrm{O}_{3}= \\
\% \mathrm{~K}_{2} \mathrm{O}=.632 \\
\text { Other \% }= \\
\text { Other \% = }
\end{array}
$$

$\% \mathrm{CaO}=7.43$

$\% \mathrm{PbO}=$

$\% \mathrm{MgO}=5.91$

$\% \mathrm{SiO}_{2}=71.3$

$K_{\mathrm{IC}}=$

$K_{\mathrm{IC}}=$

Gamma =

Gamma =

Environment $=6 \mathrm{~N} \mathrm{HCl}$

Reference = WIEDERHORN,S.M., JOHNSON,H., JACTAW, 56, 192-97, 1973

Comments $=$ 
Specific Material $=7940$

Manufacturer $=$ Corning Glass
$\% \mathrm{Al}_{2} \mathrm{O}_{3}=$
$\% \mathrm{~B}_{2} \mathrm{O}_{3}=$
$\% \mathrm{Na}_{2} \mathrm{O}=$
$\% \mathrm{~K}_{2} \mathrm{O}=$
Other $\%=$
Other Formula $=$
Other Formula $=$
Other $\%=$

$\% \mathrm{CaO}=$

$\% \mathrm{PbO}=$

$\% \mathrm{MgO}=$

$\% \mathrm{SiO}_{2}=100$

Young's Modulus =

1st Technique $=\mathrm{DCB}$

$K_{\mathrm{IC}}=.74$

$K_{\text {IC }}=.75$

Gamma $=$

2nd Technique $=\mathrm{NB}$

Gamma $=$

$n=$

Environment $=$ Vacuum

Reference $=$ WIEDERHORN,S., Frac. Mech. of Cer., Vol. 2, 829-40, 1974

Comments $=$

Specific Material $=7900$

Manufacturer $=$ Corning Glass

$\% \mathrm{Al}_{2} \mathrm{O}_{3}=.179 \quad \% \mathrm{~B}_{2} \mathrm{O}_{3}=2.62$

$\% \mathrm{Na}_{2} \mathrm{O}=$

$\% \mathrm{~K}_{2} \mathrm{O}=$

$\% \mathrm{CaO}=$

$\% \mathrm{PbO}=$

$\% \mathrm{MgO}=$

Other Formula =

Other $\%=$

Other Formula =

Other $\%=$

$\% \mathrm{SiO}_{2}=97.1$

Young's Modulus =

1st Technique $=\mathrm{DCB}$

$K_{\mathrm{IC}}=.7$

2nd Technique $=\mathrm{NB}$

$K_{\text {IC }}=.71$

Gamma $=$

$n=$

Environment $=$ Vacuum

Reference = WIEDERHORN,S., Frac. Mech. of Cer., Vol. 2, 829-40, 1974

Comments $=$

Specific Material = 7913

Manufacturer $=$ Corning Glass
$\% \mathrm{Al}_{2} \mathrm{O}_{3}=.297$
$\% \mathrm{~B}_{2} \mathrm{O}_{3}=2.61$
$\% \mathrm{Na}_{2} \mathrm{O}=$
$\% \mathrm{~K}_{2} \mathrm{O}=$
Other Formula $=$
Other $\%=$
Other Formula $=$
Other $\%=$

$\% \mathrm{CaO}=$

$\% \mathrm{PbO}=$

Gamma $=$

Young's Modulus =

1st Technique $=\mathrm{DCB}$

$K_{\mathrm{IC}}=.72$

2nd Technique $=$

$K_{\mathrm{IC}}=$

Gamma $=$

$n=$

Environment $=$ Vacuum

Reference = WIEDERHORN,S., Frac. Mech. of Cer., Vol. 2, 829-40, 1974

Comments $=$

Specific Material $=1723$

Manufacturer $=$ Corning Glass

$\% \mathrm{Al}_{2} \mathrm{O}_{3}=9.43 \quad \% \mathrm{~B}_{2} \mathrm{O}_{3}=4.60$

$\% \mathrm{Na}_{2} \mathrm{O}=$

$\% \mathrm{~K}_{2} \mathrm{O}=$

$\% \mathrm{CaO}=11.4$

$\% \mathrm{MgO}=11.1$

Other Formula $=\mathrm{BaO}$

Other Formula =

Other $\%=2.51$

$\% \mathrm{PbO}=$

$\% \mathrm{MgO}=$

$\% \mathrm{SiO}_{2}=97.0$

Young's Modulus =

1st Technique $=\mathrm{DCB}$

Other $\%=$

Gamma $=$

2nd Technique $=\mathrm{NB}$

$K_{\mathrm{IC}}=.85$

$K_{\text {IC }}=.84$

Gamma $=$

$n=$

Environment $=$ Vacuum

Reference $=$ WIEDERHORN,S., Frac. Mech. of Cer., Vol. 2, 829-40, 1974

Comments $=$ 
Specific Material $=7740$

Manufacturer $=$ Corning Glass

$\begin{array}{ll}\% \mathrm{Al}_{2} \mathrm{O}_{3}=1.21 & \% \mathrm{~B}_{2} \mathrm{O}_{3}=11.5 \\ \% \mathrm{Na}_{2} \mathrm{O}=3.98 & \% \mathrm{~K}_{2} \mathrm{O}= \\ \text { Other Formula }= & \text { Other } \%= \\ \text { Other Formula }= & \text { Other } \%=\end{array}$

$\% \mathrm{CaO}=\quad \% \mathrm{MgO}=$

$\% \mathrm{PbO}=\quad \% \mathrm{SiO}_{2}=83.2$

Young's Modulus =

1st Technique $=\mathrm{DCB}$

$K_{\mathrm{IC}}=.76$

Gamma $=$

2nd Technique $=\mathrm{NB}$

$K_{\mathrm{IC}}=.78$

Gamma $=$

$n=$

Environment $=$ Vacuum

Reference = WIEDERHORN,S., Frac. Mech. of Cer., Vol. 2, 829-40, 1974

Comments $=$

Specific Material $=$ BK-7

Manufacturer $=$ Schott

$\% \mathrm{Al}_{2} \mathrm{O}_{3}=$

$\% \mathrm{Na}_{2} \mathrm{O}=10.3$

Other Formula $=\mathrm{BaO}$

Other Formula $=\mathrm{CeO}$

Young's Modulus =

1st Technique $=\mathrm{DCB}$

2nd Technique $=\mathrm{NB}$

$n=$

Environment $=$ Vacuum

Reference = WIEDERHORN,S., Frac. Mech. of Cer., Vol. 2, 829-40, 1974

Comments $=$

Specific Material $=$ UBK-7

Manufacturer $=$ Schott

$\% \mathrm{Al}_{2} \mathrm{O}_{3}=$

$\% \mathrm{Na}_{2} \mathrm{O}=10.2$

Other Formula $=\mathrm{BaO}$

Other Formula =

Young's Modulus =

1st Technique $=\mathrm{DCB}$

2nd Technique $=$ NB

$\% \mathrm{~B}_{2} \mathrm{O}_{3}=10.0$

$\% \mathrm{~K}_{2} \mathrm{O}=4.74$

Other $\%=.833$

Other $\%=<.1 \mathrm{~W} \%$

$K_{\text {IC }}=.84$
$\% \mathrm{~B}_{2} \mathrm{O}_{3}=10.0$

$\% \mathrm{~K}_{2} \mathrm{O}=4.71$

Other \% $=.828$

Other $\%=$
$K_{\text {IC }}=.89$

$K_{\text {IC }}=.90$
$\% \mathrm{CaO}=.227$

$\% \mathrm{PbO}=$

$\% \mathrm{MgO}=$

$\% \mathrm{SiO}_{2}=73.3$

Gamma =

Gamma $=$

$n=$

$\% \mathrm{CaO}=.226$

$\% \mathrm{PbO}=$

$\% \mathrm{MgO}=$

$\% \mathrm{SiO}_{2}=73.9$

Environment $=$ Vacuum

Reference $=$ WIEDERHORN,S., Frac. Mech. of Cer., Vol. 2, 829-40, 1974

Comments $=$

Specific Material $=$ SF -1

Manufacturer $=$ Schott

$\% \mathrm{Al}_{2} \mathrm{O}_{3}=$
$\% \mathrm{Na}_{2} \mathrm{O}=7.15$

Other Formula $=$

Other Formula =

Young's Modulus =

1st Technique $=\mathrm{DCB}$

2nd Technique $=\mathrm{NB}$

$n=$

Environment $=$ Vacuum

Reference = WIEDERHORN,S., Frac. Mech. of Cer., Vol. 2, 829-40,1974

Comments $=$

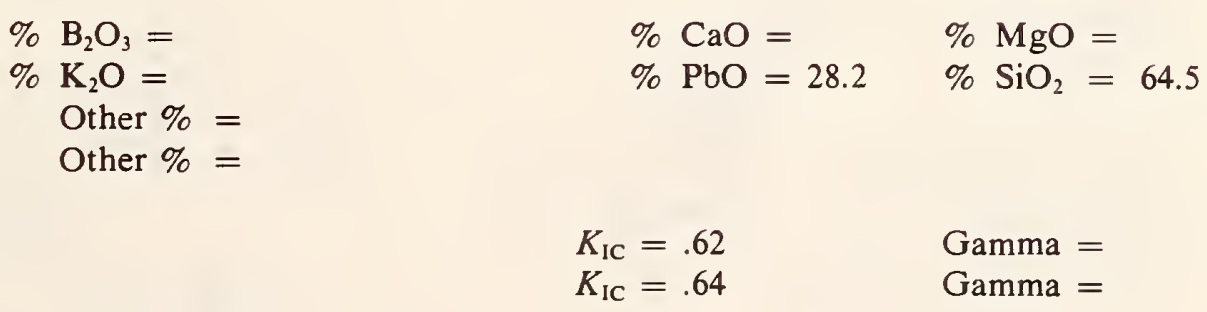

Gamma $=$

Gamma $=$ 
Specific Material $=7900$

Manufacturer $=$ Corning Glass

$\% \mathrm{Al}_{2} \mathrm{O}_{3}=.179$

$\% \mathrm{~B}_{2} \mathrm{O}_{3}=2.62$

$\% \mathrm{CaO}=$

$\% \mathrm{MgO}=$

$\% \mathrm{Na}_{2} \mathrm{O}=$

$\% \mathrm{~K}_{2} \mathrm{O}=$

$\% \mathrm{PbO}=$

$\% \mathrm{SiO}_{2}=97.1$

Other Formula =

Other $\%=$

Other Formula =

Other $\%=$

$K_{\mathrm{IC}}=$

Gamma $=$

1st Technique $=$ DCB

$K_{\mathrm{IC}}=$

Gamma $=$

2nd Technique $=$

$n=50.5$

Environment $=$ air, $100 \% \mathrm{RH}$

Reference = WIEDERHORN,S.,EVANS,A.,Frac. Mech. of Cer. 2,829-41,1974

Comments $=$

Specific Material $=1723$

Manufacturer $=$ Corning Glass

$\% \mathrm{Al}_{2} \mathrm{O}_{3}=9.43 \quad \% \mathrm{~B}_{2} \mathrm{O}_{3}=4.60$

$\% \mathrm{~K}_{2} \mathrm{O}=$

$\% \mathrm{CaO}=11.4$

$\% \mathrm{MgO}=11.1$

$\% \mathrm{Na}_{2} \mathrm{O}=$

Other $\%=2.51$

$\% \mathrm{PbO}=$

$\% \mathrm{SiO}_{2}=60.8$

Other Formula $=\mathrm{BaO}$

Other $\%=$

Young's Modulus =

1st Technique $=\mathrm{DCB}$

2nd Technique $=$

$n=46$

Environment $=$ air, $100 \% \mathrm{RH}$

Reference $=$ WIEDERHORN,S.,EVANS,A.,Frac. Mech. of Cer. 2,829-41,1974

Comments $=$

Specific Material $=\mathrm{SF}-1$

Manufacturer $=$ Schott

$\% \mathrm{Al}_{2} \mathrm{O}_{3}=$
$\% \mathrm{Na}_{2} \mathrm{O}=7.15$

$\% \mathrm{~B}_{2} \mathrm{O}_{3}=$

$\% \mathrm{CaO}=$

$K_{\text {IC }}=$

Gamma $=$

$K_{\text {IC }}=$

Gamma $=$

Other Formula $=$

Other Formula =

Young's Modulus =

$\% \mathrm{~K}_{2} \mathrm{O}=$

$\% \mathrm{PbO}=28.2$

$\% \mathrm{MgO}=$

Other $\%=$

Other $\%=$

$\% \mathrm{SiO}_{2}=64.5$

1st Technique $=$ DCB

2nd Technique $=$

$n=38$

Environment $=$ air, $100 \% \mathrm{RH}$

Reference $=$ WIEDERHORN,S.,EVANS,A.,Frac. Mech. of Cer. 2,829-41,1974

Comments $=$

\section{WIL83}

Specific Material $=$ NBP-3

Manufacturer =

$K_{\mathrm{IC}}=$

Gamma =

$K_{\mathrm{IC}}=$

Gamma =

$\% \mathrm{Al}_{2} \mathrm{O}_{3}=$

$\% \mathrm{Na}_{2} \mathrm{O}=30$

$\% \mathrm{~B}_{2} \mathrm{O}_{3}=$

$\% \mathrm{~K}_{2} \mathrm{O}=$

Other Formula $=\mathrm{BaO}$

Other $\%=20$

Other Formula $=\mathrm{P}_{2} \mathrm{O}_{5}$

Other $\%=50$

$\% \mathrm{CaO}=$

$\% \mathrm{PbO}=$

$\% \mathrm{MgO}=$

$\% \mathrm{SiO}_{2}=$

Young's Modulus =

1st Technique $=$ Short bar

2nd Technique $=$

$n=$

Environment $=$ air

Reference $=$ WILDER, J., Private Communications, 1983

Comments $=$
$K_{\mathrm{IC}}=.47$

$K_{\mathrm{IC}}=$
Gamma $=$

Gamma $=$ 
Specific Material $=$ NBP -4

Manufacturer $=$

$\% \mathrm{Al}_{2} \mathrm{O}_{3}=$

$\% \mathrm{~B}_{2} \mathrm{O}_{3}=$

$\% \mathrm{~K}_{2} \mathrm{O}=$

$\% \mathrm{CaO}=$

$\% \mathrm{MgO}=$

$\% \mathrm{Na}_{2} \mathrm{O}=25$

Other $\%=25$

$\% \mathrm{PbO}=$

$\% \mathrm{SiO}_{2}=$

Other Formula $=\mathrm{BaO}$

Other $\%=50$

Other Formula $=\mathrm{P}_{2} \mathrm{O}_{5}$
Young's Modulus $=4.3 \mathrm{E4}$

1st Technique $=$ Short bar

$K_{\mathrm{IC}}=.52$

$K_{\mathrm{IC}}=$

Gamma $=3.14$

2nd Technique $=$

$n=$

Environment $=$ air

Reference $=$ WILDER, J., Private Communications, 1983

Comments $=$ Poisson's Ratio $=.2929$

Specific Material = NBP-5

Manufacturer $=$

$\% \mathrm{Al}_{2} \mathrm{O}_{3}=$

$\% \mathrm{Na}_{2} \mathrm{O}=20$

$\% \mathrm{~B}_{2} \mathrm{O}_{3}=$

$\% \mathrm{~K}_{2} \mathrm{O}=$

Other Formula $=\mathrm{BaO}$

Other $\%=30$

Other Formula $=\mathrm{P}_{2} \mathrm{O}_{5}$

Other $\%=50$

$\% \mathrm{CaO}=$

$\% \mathrm{PbO}=$

$K_{\text {IC }}=.50$

$K_{\text {IC }}=$

1st Technique $=$ Short bar

2nd Technique $=$

$n=$

Environment $=$ air

Reference = WILDER, J., Private Communications, 1983

Comments $=$ Poisson's Ratio $=.2930$

Specific Material $=$ NBP-6

Manufacturer $=$

$\% \mathrm{Al}_{2} \mathrm{O}_{3}=$

$\% \mathrm{Na}_{2} \mathrm{O}=15$

$\% \mathrm{~B}_{2} \mathrm{O}_{3}=$

$\% \mathrm{~K}_{2} \mathrm{O}=$

Other Formula $=\mathrm{BaO}$

Other $\%=35$

Other Formula $=\mathrm{P}_{2} \mathrm{O}_{5} \quad$ Other $\%=50$

Young's Modulus $=4.5 \mathrm{E} 4$

1st Technique $=$ Short bar

2nd Technique $=$

$n=$

Environment $=$ air

Reference = WILDER, J., Private Communications, 1983

Comments $=$ Poisson's Ratio $=.2958$

Specific Material $=$ NBP-7

Manufacturer $=$

$\% \mathrm{Al}_{2} \mathrm{O}_{3}=$

$\% \mathrm{Na}_{2} \mathrm{O}=10$

$\% \mathrm{~B}_{2} \mathrm{O}_{3}=$

$\% \mathrm{~K}_{2} \mathrm{O}=$

Other Formula $=\mathrm{BaO}$

Other $\%=40$

Other Formula $=\mathrm{P}_{2} \mathrm{O}_{5} \quad$ Other $\%=50$

Young's Modulus $=4.5 \mathrm{E} 4$

1st Technique $=$ Short bar

2nd Technique $=$

$n=$

Environment $=$ air

Reference = WILDER, J., Private Communications, 1983

Comments $=$ Poisson's Ratio $=.2975$
$K_{\mathrm{IC}}=.52$

$K_{\mathrm{IC}}=$

$\% \mathrm{CaO}=$

$\% \mathrm{PbO}=$

$\% \mathrm{CaO}=$

$\% \mathrm{PbO}=$

$K_{\mathrm{IC}}=.51$

$K_{\text {IC }}=$
Gamma $=$

$\% \mathrm{MgO}=$

$\% \mathrm{SiO}_{2}=$

Gamma $=2.80$

Gamma $=$
Gamma $=3.03$

Gamma $=$
$\% \mathrm{MgO}=$

$\% \mathrm{SiO}_{2}=$$$
\text { Gamma }
$$

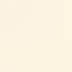


Specific Material $=$ NBP-10

Manufacturer =

$\% \mathrm{Al}_{2} \mathrm{O}_{3}=$

$\% \mathrm{Na}_{2} \mathrm{O}=30$

$\% \mathrm{~B}_{2} \mathrm{O}_{3}=$

$\% \mathrm{CaO}=$

$\% \mathrm{PbO}=$

$\% \mathrm{MgO}=$

$\% \mathrm{~K}_{2} \mathrm{O}=$

$\begin{array}{ll}\text { Other Formula }=\mathrm{BaO} & \text { Other } \%=16 \\ \text { Other Formula }=\mathrm{P}_{2} \mathrm{O}_{5} & \text { Other } \%=50\end{array}$

$\% \mathrm{SiO}_{2}=$

Young's Modulus $=4.3 E 4$

1st Technique $=$ Short bar

$K_{\text {IC }}=.47$

2nd Technique $=$

$K_{\mathrm{IC}}=$

Gamma $=2.56$

$n=$

Environment $=$ air

Reference $=$ WILDER, J., Private Communications, 1983

Comments $=3$ rd Other Oxide- $4 \%$ ZnO, Poisson's Ratio $=.2890$

Specific Material $=$ NBP-11

Manufacturer $=$

$\% \mathrm{Al}_{2} \mathrm{O}_{3}=$

$\% \mathrm{Na}_{2} \mathrm{O}=30$

$\% \mathrm{~B}_{2} \mathrm{O}_{3}=$

$\% \mathrm{CaO}=$

$\% \mathrm{PbO}=$

Gamma $=$

Other Formula $=\mathrm{BaO} \quad$ Other $\%=12$

Other Formula $=\mathrm{P}_{2} \mathrm{O}_{5} \quad$ Other $\%=50$

Young's Modulus $=4.4 E 4$

1st Technique $=$ Short bar

2nd Technique $=$

$K_{1 \mathrm{C}}=.47$

$K_{\text {IC }}=$

Gamma $=2.48$

$n=$

Environment $=$ air

Reference = WILDER, J., Private Communications, 1983

Comments $=3$ rd Other Oxide- $8 \%$ ZnO, Poisson's Ratio $=.2867$

Specific Material $=$ NBP-13

Manufacturer $=$

$\% \mathrm{Al}_{2} \mathrm{O}_{3}=$

$\% \mathrm{Na}_{2} \mathrm{O}=30$

$\% \mathrm{~B}_{2} \mathrm{O}_{3}=$

$\% \mathrm{~K}_{2} \mathrm{O}=$

Other Formula $=\mathrm{BaO}$

Other $\%=8$

Other Formula $=\mathrm{P}_{2} \mathrm{O}_{5}$

Other $\%=50$

$\% \mathrm{CaO}=$

$\% \mathrm{PbO}=$

Gamma $=$

Young's Modulus $=4.4 E 4$

1st Technique $=$ Short bar

$K_{\mathrm{IC}}=.48$

2nd Technique $=$

$K_{\mathrm{IC}}=$

Gamma $=2.61$

$n=$

Environment $=$ air

Reference $=$ WILDER, J., Private Communications, 1983

Comments $=3$ rd Other Oxide- $12 \% \mathrm{ZnO}$, Poisson's Ratio $=.2839$

Specific Material $=$ NBP-14

Manufacturer $=$

$\% \mathrm{Al}_{2} \mathrm{O}_{3}=$

$\% \mathrm{Na}_{2} \mathrm{O}=30$

Other Formula $=\mathrm{BaO}$

$\% \mathrm{~B}_{2} \mathrm{O}_{3}=$

$\% \mathrm{~K}_{2} \mathrm{O}=$

Other $\%=4$

$\% \mathrm{CaO}=$

$\% \mathrm{MgO}=$

$\% \mathrm{SiO}_{2}=$

Other Formula $=\mathrm{P}_{2} \mathrm{O}_{5}$

Other $\%=50$

$\% \mathrm{PbO}=$

$\% \mathrm{MgO}=$

$\% \mathrm{SiO}_{2}=$

Young's Modulus $=4.4 E 4$

1st Technique $=$ Short bar

$K_{\mathrm{IC}}=.47$

$K_{\text {IC }}=$

Gamma $=2.48$

2nd Technique $=$

Gamma $=$

$n=$

Environment $=$ air

Reference $=$ WILDER, J., Private Communications, 1983

Comments $=3$ rd Other Oxide- $16 \%$ ZnO, Poisson's Ratio $=.2815$ 
Specific Material $=$ NZP-1

Manufacturer =
$\% \mathrm{Al}_{2} \mathrm{O}_{3}=$
$\% \mathrm{~B}_{2} \mathrm{O}_{3}=$
$\% \mathrm{Na}_{2} \mathrm{O}=30$
$\% \mathrm{~K}_{2} \mathrm{O}=$
Other $\%=20$
Other Formula $=\mathrm{ZnO}$
Other $\%=50$

$\% \mathrm{CaO}=$

$\% \mathrm{PbO}=$

$K_{\mathrm{IC}}=.48$

$K_{\mathrm{IC}}=$
$\% \mathrm{MgO}=$

$\% \mathrm{SiO}_{2}=$

Other Formula $=\mathrm{P}_{2} \mathrm{O}_{5}$
Young's Modulus $=4.4 E 4$

1st Technique $=$ Short bar

2nd Technique $=$

$n=$

Environment $=$ air

Reference = WILDER, J., Private Communications, 1983

Comments $=$ Poisson's Ratio $=.2803$

Specific Material $=$ NBP-20

Manufacturer $=$
$\% \mathrm{Al}_{2} \mathrm{O}_{3}=$
$\% \mathrm{~B}_{2} \mathrm{O}_{3}=$
$\% \mathrm{Na}_{2} \mathrm{O}=30$
$\% \mathrm{~K}_{2} \mathrm{O}=$
Other Formula $=\mathrm{BaO}$
Other $\%=16$
Other Formula $=\mathrm{P}_{2} \mathrm{O}_{5}$
Other $\%=50$

$\% \mathrm{CaO}=4$

$\% \mathrm{PbO}=$

$K_{\mathrm{IC}}=.52$

$K_{\mathrm{IC}}=$

Gamma $=2.63$

Gamma $=$

Young's Modulus $=4.3 E 4$

1st Technique $=$ Short bar

2nd Technique $=$

$n=$

Environment $=$ air

Reference = WILDER, J., Private Communications, 1983

Comments $=$ Poisson's Ratio $=.2886$

Specific Material $=$ NBP-21

Manufacturer $=$

$\% \mathrm{Al}_{2} \mathrm{O}_{3}=$

$\% \mathrm{Na}_{2} \mathrm{O}=30$

Other Formula $=\mathrm{BaO}$

Other Formula $=\mathrm{P}_{2} \mathrm{O}_{5}$

Young's Modulus $=4.4 E 4$

1st Technique $=$ Short bar

2nd Technique $=$

$n=$

Environment $=$ air

Reference = WILDER, J., Private Communications, 1983

Comments $=$ Poisson's Ratio $=.2855$

Specific Material $=$ NBP -23

Manufacturer $=$

$\% \mathrm{Al}_{2} \mathrm{O}_{3}=$

$\% \mathrm{Na}_{2} \mathrm{O}=30$

$\% \mathrm{~B}_{2} \mathrm{O}_{3}=$

$\% \mathrm{~K}_{2} \mathrm{O}=$

Other Formula $=\mathrm{BaO}$

Other $\%=8$

Other Formula $=\mathrm{P}_{2} \mathrm{O}_{5}$

Young's Modulus $=4.5 E 4$

1st Technique $=$ Short bar

2nd Technique $=$

$n=$

Environment $=$ air

Reference = WILDER, J., Private Communications, 1983

Comments $=$ Poisson's Ratio $=.2829$
$\% \mathrm{CaO}=8$

$\% \mathrm{PbO}=$

$K_{\mathrm{IC}}=.54$

$K_{\mathrm{IC}}=$

$\% \mathrm{CaO}=12$

$\% \mathrm{PbO}=$

$K_{\mathrm{IC}}=.52$

$K_{\text {IC }}=$
$\% \mathrm{MgO}=$

$\% \mathrm{SiO}_{2}=$

Gamma $=3.18$

Gamma $=$
$\% \mathrm{MgO}=$

$\% \mathrm{SiO}_{2}=$

Gamma $=3.29$

Gamma = 
Specific Material $=$ NBP-24

Manufacturer $=$
$\% \mathrm{Al}_{2} \mathrm{O}_{3}=$
$\% \mathrm{Na}_{2} \mathrm{O}=30$
$\% \mathrm{~B}_{2} \mathrm{O}_{3}=$
$\% \mathrm{~K}_{2} \mathrm{O}=$
Other Formula $=\mathrm{BaO}$
Other $\%=4$
Other Formula $=\mathrm{P}_{2} \mathrm{O}_{5}$
Other $\%=50$

$$
\begin{array}{ll}
\% \mathrm{CaO}=16 & \% \mathrm{MgO}= \\
\% \mathrm{PbO}= & \% \mathrm{SiO}_{2}=
\end{array}
$$

$K_{\mathrm{IC}}=.53$

$K_{\mathrm{IC}}=$

Gamma $=3.01$

Gamma $=$

2nd Technique $=$

$n=$

Environment $=$ air

Reference $=$ WILDER, J., Private Communications, 1983

Comments $=$ Poisson's Ratio $=.2801$

Specific Material $=$ NCP-6

Manufacturer $=$

$\% \mathrm{Al}_{2} \mathrm{O}_{3}=$

$\% \mathrm{Na}_{2} \mathrm{O}=30$

$\% \mathrm{~B}_{2} \mathrm{O}_{3}=$

$\% \mathrm{~K}_{2} \mathrm{O}=$

Other $\%=50$

Other $\%=$

Other Formula $=$

Young's Modulus $=4.7 E 4$

1st Technique $=$ Short bar

2nd Technique $=$

$n=$

Environment $=$ air

Reference $=$ WILDER, J., Private Communications, 1983

Comments $=$ Poisson's Ratio $=.2757$

Specific Material $=$ NCP-9

Manufacturer $=$

$\% \mathrm{Al}_{2} \mathrm{O}_{3}=$

$\% \mathrm{Na}_{2} \mathrm{O}=30$

Other Formula $=\mathrm{ZnO}$

$\% \mathrm{~B}_{2} \mathrm{O}_{3}=$

$\% \mathrm{~K}_{2} \mathrm{O}=$

Other $\%=4$

Other Formula $=\mathrm{P}_{2} \mathrm{O}_{5}$

Other $\%=50$

Young's Modulus $=4.6 E 4$

1st Technique $=$ Short bar

2nd Technique $=$

$n=$

Environment $=$ air

Reference $=$ WILDER, J., Private Communications, 1983

Comments $=$ Poisson's Ratio $=.2768$

Specific Material $=$ NCP -10

Manufacturer $=$

$\% \mathrm{Al}_{2} \mathrm{O}_{3}=$

$\% \mathrm{Na}_{2} \mathrm{O}=30$

$\% \mathrm{~B}_{2} \mathrm{O}_{3}=$

$\% \mathrm{~K}_{2} \mathrm{O}=$

Other Formula $=\mathrm{ZnO}$

Other $\%=8$

Other Formula $=\mathrm{P}_{2} \mathrm{O}_{5}$

Other $\%=50$

Young's Modulus $=4.6 E 4$

1st Technique $=$ Short bar

2nd Technique $=$

$n=$

Environment $=$ air

Reference $=$ WILDER, J., Private Communications, 1983

Comments $=$ Poisson's Ratio $=.2768$

$\begin{array}{ll}\% \mathrm{CaO}=20 & \% \mathrm{MgO}= \\ \% \mathrm{PbO}= & \% \mathrm{SiO}_{2}=\end{array}$

$K_{\mathrm{IC}}=.46$

$K_{\mathrm{IC}}=$

Gamma $=2.23$

Gamma $=$
$\% \mathrm{CaO}=16$

$\% \mathrm{PbO}=$

$K_{\mathrm{IC}}=.46$

$K_{1 \mathrm{C}}=$

$\% \mathrm{CaO}=12$

$\% \mathrm{PbO}=$

$K_{\mathrm{lC}}=.45$

$K_{\mathrm{IC}}=$
$\% \mathrm{MgO}=$

$\% \mathrm{SiO}_{2}=$

Gamma $=2.27$

Gamma $=$ 
Specific Material $=\mathrm{NCP}-12$

Manufacturer =

$\% \mathrm{Al}_{2} \mathrm{O}_{3}=$

$\% \mathrm{Na}_{2} \mathrm{O}=30$

Other Formula $=\mathrm{ZnO}$

$\% \mathrm{~B}_{2} \mathrm{O}_{3}=$

$\% \mathrm{~K}_{2} \mathrm{O}=$

Other $\%=16$

Other Formula $=\mathrm{P}_{2} \mathrm{O}_{5}$

Other $\%=50$

Young's Modulus $=4.5 E 4$

1st Technique $=$ Short bar

2nd Technique $=$

$n=$

Environment $=$ air

Reference $=$ WILDER, J., Private Communications, 1983

Comments $=$ Poisson's Ratio $=.2788$

Specific Material $=$ NBP-1

Manufacturer =

$\% \mathrm{Al}_{2} \mathrm{O}_{3}=$

$\% \mathrm{Na}_{2} \mathrm{O}=40$

$\% \mathrm{~B}_{2} \mathrm{O}_{3}=$

$\% \mathrm{~K}_{2} \mathrm{O}=$

Other Formula $=\mathrm{BaO}$

Other $\%=10$

Other Formula $=\mathrm{P}_{2} \mathrm{O}_{5}$

Other $\%=50$

Young's Modulus $=4.0 \mathrm{E} 4$

1st Technique $=$ Short bar

2nd Technique $=$

$n=$

Environment $=$ air

Reference = WILDER, J., Private Communications, 1983

Comments $=$ Poisson's Ratio $=.2909$

Specific Material $=$ NBP-2

Manufacturer $=$

$\% \mathrm{Al}_{2} \mathrm{O}_{3}=$

$\% \mathrm{Na}_{2} \mathrm{O}=35$

$\% \mathrm{~B}_{2} \mathrm{O}_{3}=$

$\% \mathrm{~K}_{2} \mathrm{O}=$

Other Formula $=\mathrm{BaO}$

Other $\%=15$

Other Formula $=\mathrm{P}_{2} \mathrm{O}_{5}$

Other $\%=50$

$K_{\mathrm{IC}}=.46$

$\% \mathrm{CaO}=4$

$\% \mathrm{PbO}=$

$\% \mathrm{MgO}=$

$\% \mathrm{SiO}_{2}=$

$K_{\mathrm{IC}}=$

Gamma $=2.35$

$\% \mathrm{CaO}=$

$\% \mathrm{PbO}=$

$\% \mathrm{MgO}=$

$\% \mathrm{SiO}_{2}=$

$K_{\mathrm{IC}}=.44$

$K_{\mathrm{IC}}=$

Gamma $=2.39$

Gamma $=$

$\% \mathrm{CaO}=$

$\% \mathrm{PbO}=$

$\% \mathrm{MgO}=$

$\% \mathrm{SiO}_{2}=$

Young's Modulus = $4.1 \mathrm{E} 4$

1st Technique $=$ Short bar

2nd Technique $=$

$n=$

Environment $=$ air

Reference $=$ WILDER, J., Private Communications, 1983

Comments $=$ Poisson's Ratio $=.2916$

$K_{\mathrm{IC}}=.46$

$K_{\mathrm{IC}}=$
Gamma $=2.60$

Gamma = 
Table 4. Compositions of silicate base glasses

\begin{tabular}{|c|c|c|c|c|c|c|c|c|c|}
\hline $\mathrm{SiO}_{2}$ & $\mathrm{Al}_{2} \mathrm{O}_{3}$ & $\mathrm{~B}_{2} \mathrm{O}_{3}$ & $\mathrm{CaO}$ & $\mathrm{K}_{2} \mathrm{O}$ & $\mathrm{MgO}$ & $\mathrm{Na}_{2} \mathrm{O}$ & $\mathrm{PbO}$ & Other & Ref. \\
\hline 100 & & & & & & & & & MEC74 \\
\hline 100 & & & & & & & & & KEN74 \\
\hline 100 & & & & & & & & & WIE74/1 \\
\hline 100 & & & & & & & & & PRO67 \\
\hline 100 & & & & & & & & & TAR78 \\
\hline 100 & & & & & & & & & RIT71 \\
\hline 99.9 & & & & & & & & & WIE69 \\
\hline 99.9 & & & & & & & & & WIE70 \\
\hline 97.1 & .179 & 2.62 & & & & & & & WIE $74 / 2$ \\
\hline 97.1 & .179 & 2.62 & & & & & & & WIE74/1 \\
\hline 97.0 & .297 & 2.61 & & & & & & & WIE74/1 \\
\hline 96.7 & .594 & 2.61 & & & & & & & WIE69 \\
\hline 92.5 & & & & & & 7.5 & & & VER80 \\
\hline 87.5 & & & & & & 12.5 & & & VER80 \\
\hline 83.2 & 1.21 & 11.5 & & & & 3.98 & & & WIE74/1 \\
\hline 83 & & & & & & 17 & & & KEN74 \\
\hline 82.3 & 1.21 & 12.4 & & & & 3.99 & & & WIE69 \\
\hline 82.3 & 1.21 & 12.4 & & & & 3.99 & & & RIT75 \\
\hline 82.3 & 1.21 & 12.4 & & & & 3.99 & & & RIT73 \\
\hline 82.3 & 1.21 & 12.4 & & & & 3.99 & & f & RIT71 \\
\hline 82.3 & 1.21 & 12.4 & & & & 3.99 & & & WIE70 \\
\hline 82.0 & 3.36 & 11.3 & & & & 3.26 & & & MEC74 \\
\hline 80.0 & & & 5.36 & & & 14.5 & & & MIY83 \\
\hline 80 & & & & & & 20 & & & VER 80 \\
\hline 80 & & & & & & 20 & & & KEN74 \\
\hline 80 & & & & & & 20 & & & EAG78 \\
\hline 76 & & & & & & & 24 & & VER 80 \\
\hline 75.6 & 2.97 & & & 1.60 & & 12.2 & 7.59 & & WIE69 \\
\hline 75.6 & 2.97 & & & 1.60 & & 12.2 & 7.59 & & WIE70 \\
\hline 75.1 & & & 5.37 & & & 19.4 & & & MIY83 \\
\hline 75 & & & & & & 25 & & & KEN74 \\
\hline 75 & & 18.8 & & & & 6.2 & & & SWE78 \\
\hline 75 & & 14.2 & & & & 10.8 & & & SWE78 \\
\hline 75 & & 10 & & & & 15 & & & SWE78 \\
\hline 75 & & 4.2 & & & & 20.8 & & & SWE78 \\
\hline 75 & & & & & & 25 & & & SIM81 \\
\hline 75 & 5 & & & & & 20 & & & EAG78 \\
\hline 73.9 & & 10.0 & .226 & 4.71 & & 10.2 & & .828 & WIE74/1 \\
\hline 73.3 & & 10.0 & .227 & 4.74 & & 10.3 & & $<1.0 \mathrm{~W} \%$ & WIE74/1 \\
\hline 73.0 & 18.8 & 8.08 & & & & & & & FRE83/1 \\
\hline 73 & & & & & & & 27 & & VER80 \\
\hline 71.9 & 1.76 & & 5.35 & & 4.46 & 16.4 & & & CHA58 \\
\hline 71.9 & .581 & & 5.28 & & 5.88 & 16.2 & & & MEC74 \\
\hline 71.4 & 1.75 & & 7.44 & & 5.91 & 13.4 & & & SIM81 \\
\hline 71.3 & 1.16 & & 7.43 & .632 & 5.91 & 13.4 & & & WIE69 \\
\hline 71.3 & 1.16 & & 7.43 & .632 & 5.91 & 13.4 & & & BRU77 \\
\hline 71.3 & 1.16 & & 7.43 & .632 & 5.91 & 13.4 & & & MOU59 \\
\hline 71.3 & 1.16 & & 7.43 & .632 & 5.91 & 13.4 & & & RIT69 \\
\hline 71.3 & 1.16 & & 7.43 & .632 & 5.91 & 13.4 & & & WIE73 \\
\hline 71.3 & 1.16 & & 7.43 & .632 & 5.91 & 13.4 & & & WIE70 \\
\hline 71.3 & 1.16 & & 7.43 & .632 & 5.91 & 13.4 & & & FRE83/1 \\
\hline 71.2 & 5.72 & 7.45 & 2.31 & 3.44 & & 9.41 & & $<1.0 \mathrm{~W} \%$ & FRE83/1 \\
\hline 71 & & & & & & 29 & & & KEN74 \\
\hline 70.2 & & & 5.37 & & & 24.3 & & & MIY83 \\
\hline 70 & & 23 & & & & 7 & & & SIM81 \\
\hline 70 & & 10 & & & & 20 & & & VER80 \\
\hline 70 & & & & & & 30 & & & VER80 \\
\hline 70 & 10 & & & & & 20 & & & EAG78 \\
\hline 68.8 & 1.78 & 1.74 & 5.42 & .645 & 6.03 & 14.7 & & .793 & RIT75 \\
\hline 68.8 & 1.78 & 1.74 & 5.42 & .645 & 6.03 & 14.7 & & .793 & RIT71 \\
\hline 67 & & & & & & & & 33 & MEC77 \\
\hline 67 & & & & & & 33 & & & KEN74 \\
\hline 67 & & & & & & 33 & & & SIM81 \\
\hline 67 & & & & & & & & 33 & FRE83/2 \\
\hline
\end{tabular}


Table 4. Compositions of silicate base glasses - Continued

\begin{tabular}{|c|c|c|c|c|c|c|c|c|c|}
\hline $\mathrm{SiO}_{2}$ & $\mathrm{Al}_{2} \mathrm{O}_{3}$ & $\mathrm{~B}_{2} \mathrm{O}_{3}$ & $\mathrm{CaO}$ & $\mathrm{K}_{2} \mathrm{O}$ & $\mathrm{MgO}$ & $\mathrm{Na}_{2} \mathrm{O}$ & $\mathrm{PbO}$ & Other & Ref. \\
\hline 66.6 & 10.8 & & .462 & 2.33 & 5.79 & 13.0 & & $<1.0 \mathrm{~W} \%$ & WIE70 \\
\hline 65.3 & & & 5.38 & & & 29.2 & & & MIY83 \\
\hline 64.5 & & & & & & 7.15 & 28.2 & & WIE $74 / 1$ \\
\hline 64.5 & & & & & & 7.15 & 28.2 & & WIE $74 / 2$ \\
\hline 62.5 & & & & & & & & 37.5 & MEC77 \\
\hline 62 & & & & & & 38 & & & VER 80 \\
\hline 61.4 & 9.83 & & 21.3 & & 7.37 & & & & MET72 \\
\hline 60.8 & 9.43 & 4.60 & 11.4 & & 11.1 & & & 2.51 & MEC74 \\
\hline 60.8 & 9.43 & 4.60 & 11.4 & & 11.1 & & & 2.51 & WIE74/1 \\
\hline 60.8 & 9.43 & 4.60 & 11.4 & & 11.1 & & & 2.51 & WIE74/2 \\
\hline 60 & & 20 & & & & 20 & & & VER80 \\
\hline 60 & & & & & & & 40 & & VER80 \\
\hline 60 & & 22.8 & & & & 17.2 & & & EAG78 \\
\hline 60 & & 20 & & & & 20 & & & EAG78 \\
\hline 60 & & 16.2 & & & & 23.8 & & & EAG78 \\
\hline 60 & & 8 & & & & 32 & & & EAG78 \\
\hline 60 & & & & & & 40 & & & EAG78 \\
\hline 60 & 22.8 & & 17.2 & & & & & & EAG78 \\
\hline 60 & 20 & & 20 & & & & & & EAG78 \\
\hline 60 & 13.3 & & 26.7 & & & & & & EAG78 \\
\hline 60 & 8 & & 32 & & & & & & EAG78 \\
\hline 60 & 20 & & & & & 20 & & & EAG78 \\
\hline 60 & 22.8 & & & & & 17.2 & & & EAG78 \\
\hline 60 & 20 & & & & & 20 & & & EAG78 \\
\hline 60 & 16.2 & & & & & 23.8 & & & EAG78 \\
\hline 60 & 8 & & & & & 32 & & & EAG78 \\
\hline 60 & & & & & & 40 & & & EAG78 \\
\hline 58.4 & 12.0 & 3.54 & 6.59 & & 18.3 & .994 & & & WIE69 \\
\hline 58.4 & 12.0 & 3.54 & 6.59 & & 18.3 & .994 & & & WIE70 \\
\hline 57.2 & 8.88 & 8.52 & 18.3 & & 6.97 & & & & MET72 \\
\hline 56.9 & 8.94 & 8.54 & 18.4 & & 7.04 & & & & MET72 \\
\hline 56.7 & 8.89 & 8.53 & 18.3 & & 6.97 & .504 & & & MET72 \\
\hline 56 & 24 & & & & & 20 & & & EAG78 \\
\hline 55 & & & & & & 45 & & & VER 80 \\
\hline 54.8 & 8.61 & 8.23 & 17.8 & 3.64 & 6.79 & & & & MET72 \\
\hline 54.4 & 8.55 & 8.17 & 17.6 & 2.21 & 6.74 & 2.14 & & & MET72 \\
\hline 53.8 & 8.45 & 8.07 & 17.4 & & 6.66 & 5.44 & & & MET72 \\
\hline 50 & & 30 & & & & 20 & & & VER80 \\
\hline
\end{tabular}


Table 5. Compositions of borate base glasses

\begin{tabular}{|c|c|c|c|c|c|c|c|c|c|}
\hline $\mathrm{B}_{2} \mathrm{O}_{3}$ & $\mathrm{Al}_{2} \mathrm{O}_{3}$ & $\mathrm{CaO}$ & $\mathrm{K}_{2} \mathrm{O}$ & $\mathrm{MgO}$ & $\mathrm{Na}_{2} \mathrm{O}$ & $\mathrm{PbO}$ & $\mathrm{SiO}_{2}$ & Other & Ref. \\
\hline 100 & & & & & & & & & VER 80 \\
\hline 100 & & & & & & & & & MIY80 \\
\hline 100 & & & & & & & & & MIY81 \\
\hline 99 & & & & & 1 & & & & VER 80 \\
\hline 98.4 & & & & & & 1.5 & & & MIY 80 \\
\hline 98.4 & & & & & & 1.5 & & & MIY81 \\
\hline 98 & & & & & 2 & & & & VER 80 \\
\hline 96.8 & & & & & & 3.13 & & & MIY 80 \\
\hline 96.8 & & & & & & 3.13 & & & MIY81 \\
\hline 95.8 & & & & & & 4.16 & & & MIY 80 \\
\hline 95.8 & & & & & & 4.16 & & & MIY81 \\
\hline 95 & & & & & 5 & & & & VER 80 \\
\hline 94.3 & & & & & & 5.62 & & & MIY80 \\
\hline 94.3 & & & & & & 5.62 & & & MIY81 \\
\hline 90 & & & & & 10 & & & & VER 80 \\
\hline 87.9 & & & & & & 12.0 & & & MIY 80 \\
\hline 87.9 & & & & & & 12.0 & & & MIY81 \\
\hline 85.9 & & & & & & 14.0 & & & MIY80 \\
\hline 85.9 & & & & & & 14.0 & & & MIY81 \\
\hline 83.7 & & & & & & 16.2 & & & MIY80 \\
\hline 83.7 & & & & & & 16.2 & & & MIY81 \\
\hline 82 & & & & & & 18 & & & VER 80 \\
\hline 80.7 & & & & & & 19.2 & & & MIY 80 \\
\hline 80.7 & & & & & & 19.2 & & & MIY81 \\
\hline 80 & & & & & 20 & & & & VER80 \\
\hline 75 & & & & & 25 & & & & VER 80 \\
\hline 72 & & & & & & 28 & & & VER80 \\
\hline 70 & & & & & 20 & & 10 & & VER 80 \\
\hline 70 & & & & & & 15 & & 15 & SHI79 \\
\hline 70 & & & & & & 22.5 & & 7.5 & SHI79 \\
\hline 70 & & & & & & 30 & & & SHI79 \\
\hline 66 & & & & & 34 & & & & VER 80 \\
\hline 65.5 & & & & & & 34.5 & & & VER 80 \\
\hline 65 & & & & & 35 & & & & VER 80 \\
\hline 60 & & & & & 20 & & 20 & & VER 80 \\
\hline 60 & & & & & & 10 & & 30 & SHI79 \\
\hline 60 & & & & & & 20 & & 20 & SHI79 \\
\hline 60 & & & & & & 30 & & 10 & SHI79 \\
\hline 60 & & & & & & 40 & & & SHI79 \\
\hline 50 & & & & & 20 & & 30 & & VER 80 \\
\hline 50 & & & & & & & & 50 & VER 80 \\
\hline 50 & & & & & & 50 & & & VER80 \\
\hline 50 & & & & & & & & 50 & SHI79 \\
\hline 50 & & & & & & 12.5 & & 37.5 & SHI79 \\
\hline 50 & & & & & & 25 & & 25 & SHI79 \\
\hline 50 & & & & & & 37.5 & & 12.5 & SHI79 \\
\hline 50 & & & & & & 50 & & & SHI79 \\
\hline 45 & & & & & & & & 55 & VER80 \\
\hline 40 & & & & & 20 & & 40 & & VER 80 \\
\hline 40 & & & & & & & & 60 & VER 80 \\
\hline 40 & & & & & & 60 & & & VER80 \\
\hline 40 & & & & & & & & 60 & SHI79 \\
\hline 40 & & & & & & 15 & & 45 & SHI79 \\
\hline 40 & & & & & & 30 & & 30 & SHI79 \\
\hline 40 & & & & & & 45 & & 15 & SHI79 \\
\hline 40 & & & & & & 60 & & & SHI79 \\
\hline 30 & & & & & & 35 & & 35 & SHI79 \\
\hline 30 & & & & & & 52.5 & & 17.5 & SHI79 \\
\hline 30 & & & & & & 70 & & & SHI79 \\
\hline
\end{tabular}


Table 6. Compositions of phosphate base glasses

\begin{tabular}{|c|c|c|c|c|c|c|c|c|c|}
\hline $\mathrm{P}_{2} \mathrm{O}_{5}$ & $\mathrm{Al}_{2} \mathrm{O}_{3}$ & $\mathrm{BaO}$ & $\mathrm{CaO}$ & $\mathrm{K}_{2} \mathrm{O}$ & $\mathrm{La}_{2} \mathrm{O}_{3}$ & $\mathrm{MgO}$ & $\mathrm{Na}_{2} \mathrm{O}$ & Other & Ref. \\
\hline 75.3 & 9.4 & & & 9.4 & 1.6 & & & 4.3 & FRE84 \\
\hline 74.6 & & & & & 25 & & & 0.5 & FRE84 \\
\hline 74.6 & 25 & & & & & & & 0.5 & FRE84 \\
\hline 74.6 & 25 & & & & & & & 0.4 & FRE84 \\
\hline 70.6 & 9.4 & & & 14.1 & 1.6 & & & 4.2 & FRE84 \\
\hline 70 & & & & & & 20 & & 10 & FRE84 \\
\hline 69 & 15 & & & & & 15 & & 2 & FRE84 \\
\hline 65.9 & 18.8 & & & 9.4 & 1.7 & & & 4.2 & FRE84 \\
\hline 65.9 & 9.4 & & & 18.8 & 1.7 & & & 4.2 & FRE84 \\
\hline 65.1 & 16.5 & & & 12.5 & 1.7 & & & 4.2 & FRE84 \\
\hline 65 & & & & 12 & & 12 & & 12 & FRE84 \\
\hline 62.7 & 15.9 & & & 15.9 & 1.4 & & & 4.1 & FRE84 \\
\hline 62 & & & & 25 & & & & 13 & FRE84 \\
\hline 59 & 11.8 & & & 23.6 & 1.5 & & & 4.1 & FRE84 \\
\hline 56.7 & 9.4 & & & 28.3 & 1.5 & & & 4.0 & FRE84 \\
\hline 50 & & 8 & & & & & 30 & 12 & WIL83 \\
\hline 50 & & 8 & 12 & & & & 30 & & WIL 83 \\
\hline 50 & & & 12 & & & & 30 & 8 & WIL83 \\
\hline 50 & & & 20 & & & & 30 & & WIL83 \\
\hline 50 & & 10 & & & & & 40 & & WIL83 \\
\hline 50 & & 12 & & & & & 30 & 8 & WIL83 \\
\hline 50 & & 12 & 8 & & & & 30 & & WIL83 \\
\hline 50 & & 15 & & & & & 35 & & WIL 83 \\
\hline 50 & & 16 & & & & & 30 & 4 & WIL 83 \\
\hline 50 & & 16 & 4 & & & & 30 & & WIL 83 \\
\hline 50 & & & 4 & & & & 30 & 16 & WIL83 \\
\hline 50 & & 20 & & & & & 30 & & WIL83 \\
\hline 50 & & & & & & & 30 & 20 & WIL83 \\
\hline 50 & & 25 & & & & & 25 & & WIL83 \\
\hline 50 & & 30 & & & & & 20 & & WIL83 \\
\hline 50 & & 35 & & & & & 15 & & WIL 83 \\
\hline 50 & & 4 & & & & & 30 & 16 & WIL83 \\
\hline 50 & & 4 & 16 & & & & 30 & & WIL 83 \\
\hline 50 & & & 16 & & & & 30 & 4 & WIL83 \\
\hline 50 & & 40 & & & & & 10 & & WIL83 \\
\hline 50 & & & & & & & & 50 & ASH82 \\
\hline 50 & & 50 & & & & & & & ASH 82 \\
\hline 50 & & & & & & & & 50 & ASH 82 \\
\hline 50 & & & & & & & & 50 & ASH 82 \\
\hline 50 & & & 50 & & & & & & ASH 82 \\
\hline 50 & & & & & & 50 & & & ASH 82 \\
\hline 50 & & & & & & & 50 & & ASH82 \\
\hline
\end{tabular}


Table 7. Compositions of germanate base glasses

\begin{tabular}{llllr}
\hline \hline $\mathrm{GeO}_{2}$ & $\mathrm{~K}_{2} \mathrm{O}$ & $\mathrm{Na}_{2} \mathrm{O}$ & $\mathrm{PbO}$ & Ref. \\
\hline 100 & & & & VER80 \\
100 & 2.5 & & SME83 \\
97.5 & & 2.5 & SME83 \\
97.5 & & 4 & SME83 \\
96 & 5 & & VER80 \\
95 & & 5 & SME83 \\
95 & & & SME83 \\
95 & 7.5 & 7.5 & SME83 \\
92.5 & & 9 & SME83 \\
92.5 & 9.9 & SME83 \\
91 & & VER80 \\
90.1 & & 10 & & SME83 \\
90 & 10 & 10 & SME83 \\
90 & & & SME83 \\
90 & & & VER80 \\
88 & & & VER80 \\
87.5 & 12.5 & SME83 \\
87.5 & & & SME83 \\
86 & 14 & & SME83 \\
85 & & & SME83 \\
85 & & & SME83 \\
83.5 & 12.5 & & SME83 \\
82.5 & & & SME83 \\
80 & & & SME83 \\
63 & 15 & 37 & VER80 \\
\hline
\end{tabular}




\section{Discussion of Results}

One of the primary decisions in collecting this data was what tests represented valid measurements of $K_{\mathrm{IC}}$ or $\gamma$. Generally almost all published data, regardless of the test procedure, was included. It must be noted, however, that the there will not necessarily be a correspondence in $K_{\text {IC }}$ (or $\gamma$ ) values between different techniques. $K_{\mathrm{IC}}$ is at present an experimentally defined parameter, and historically has been taken as the stress intensity value at which a crack is moving at some rapid, though not precisely defined, rate. It can be expected that in all of these oxide glasses, cracks will grow at $K_{I}$ 's below $K_{\text {IC }}$ because of the interaction of water with the crack tip bonds under stress in the test environment. $K_{\mathrm{IC}}$ is actually just one point on a $V-K_{\mathrm{I}}$ curve. For this reason, the measured value of $K_{\mathrm{IC}}$ will be sensitive to loading rate, test environments, and the crack length dependence of $K_{\mathrm{I}}$ in the test specimen. Given these considerations, it is understandable why there is a large scatter in the $K_{\mathrm{IC}}$ and $\gamma$ data for similar glass compositions. In addition, data obtained by the notched beam technique in which the specimens were not precracked is questionable. Experience has shown that the fracture toughness data for glasses obtained in this way will lie at $K_{\mathrm{I}}$ or $\gamma$ values higher than would be obtained if a crack had been present. Nevertheless, we have included data obtained in this way because it did provide trends otherwise unobtainable.

Despite the problems associated with the wide variability in the fracture toughness data, it is still useful to consider the correlations that having this large body of data makes possible. As noted earlier, $K_{\text {IC }}$ or $\gamma$ can be plotted as a function of the mole percent of a particular oxide for a given family of glass or as a function of Young's modulus. The examples presented indicate a number of interesting observations.

Figure 1 shows a plot of $K_{\mathrm{IC}}$ as a function of the mole percent $\mathrm{Na}_{2} \mathrm{O}$ in all glasses containing both $\mathrm{Na}_{2} \mathrm{O}$ and $\mathrm{SiO}_{2}$; other constituents are present in most of these glasses in smaller quantities. It can be seen that there is no dependence of $K_{\mathrm{IC}}$ on $\mathrm{Na}_{2} \mathrm{O}$ content. Using this same data set, but plotting $\gamma$ versus Young's modulus (fig. 2) provides an entirely different picture. Here we see that there is a distinct minimum in $\gamma$ at an $E \cong 72$ $\mathrm{GPa}$. If we plot $K_{\mathrm{IC}}(=2 E \gamma)$ versus $E$ (fig. 3$)$, we see that there is a trend to increasing $K_{\mathrm{IC}}$ with increasing Young's modulus. This latter trend might be expected if Young's modulus is considered to be in part a measure of the strength as well as the stiffness of the $\mathrm{Si}-\mathrm{O}$ bond in silicate glasses.

Figure 4 shows a trend of $K_{\mathrm{IC}}$ for $\mathrm{Al}_{2} \mathrm{O}_{3}-\mathrm{P}_{2} \mathrm{O}_{5}$ glasses as a function of Young's modulus similar to that seen in the $\mathrm{Na}_{2} \mathrm{O}-\mathrm{SiO}_{2}$ series. The plot of $K_{\mathrm{IC}}$ versus the mole $\% \mathrm{P}_{2} \mathrm{O}_{5}$ (fig. 5) indicates that $K_{\mathrm{IC}}$ rises with an increase in the amount of glass former.

Figure 6 shows a plot in which $K_{\mathrm{IC}}$ increases with the mole $\%$ of $\mathrm{B}_{2} \mathrm{O}_{3}$ for all $\mathrm{B}_{2} \mathrm{O}_{3}$ glasses. However, one must be cautious in analyzing this data since many of the investigators reporting this data discuss the fact that phase separation was observed in their glass systems especially at high $\mathrm{B}_{2} \mathrm{O}_{3}$ contents. The existence of two phases in the glass will likely contribute to increases in $K_{\mathrm{IC}}$ above those for single phase glasses. These microstructural effects may, in fact, explain the relatively large values of $K_{\mathrm{IC}}$ observed in figure 7 at low values of Young's modulus. 


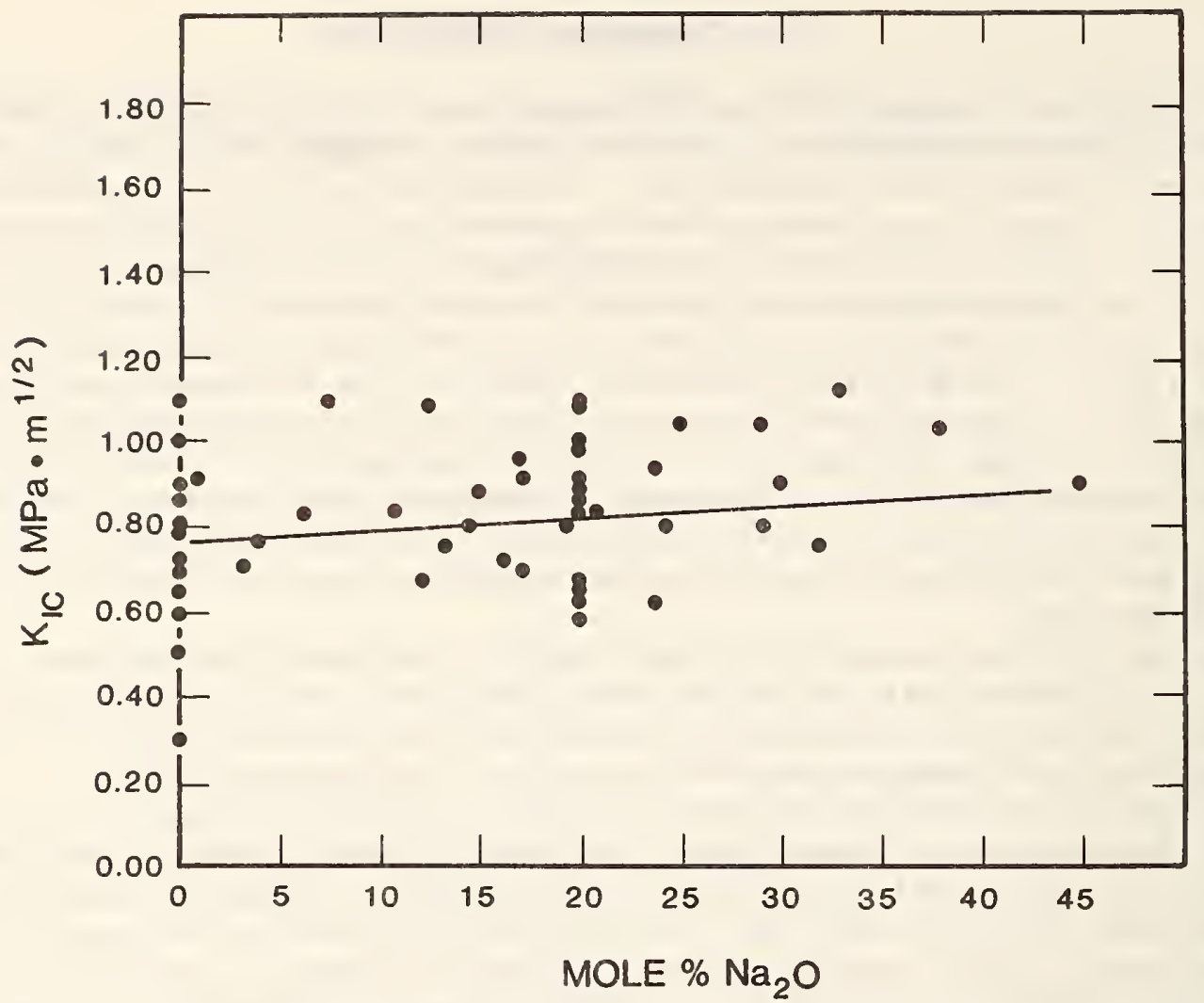

Figure 1. Critical fracture toughness, $\mathrm{K}_{\mathrm{IC}}$ plotted as a function of the mole $\% \mathrm{Na}_{2} \mathrm{O}$ for all glasses containing both $\mathrm{Na}_{2} \mathrm{O}$ and $\mathrm{SiO}{ }_{2} . \mathrm{The}$ line is the best fit curve to a quadratic equation for $\mathrm{K}_{\mathrm{IC}}$ in terms of the mole $\% \mathrm{Na}_{2} \mathrm{O}$.

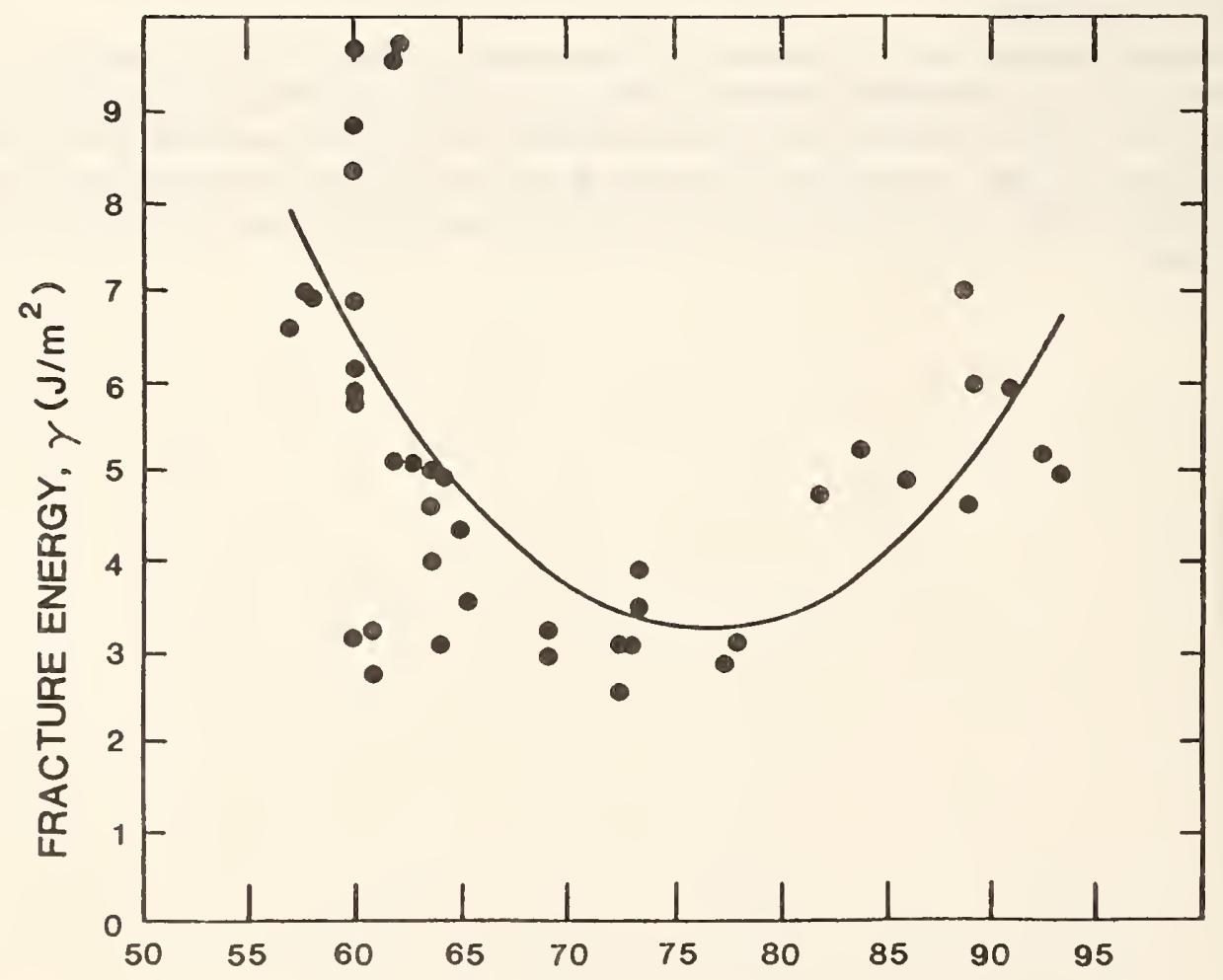

Figure 2. The same data as in figure I, plotted as $\gamma$ versus the Young's modulus of each glass. The curve is the best fit to a quadratic equation for $\gamma$ in terms of Young's modulus. 


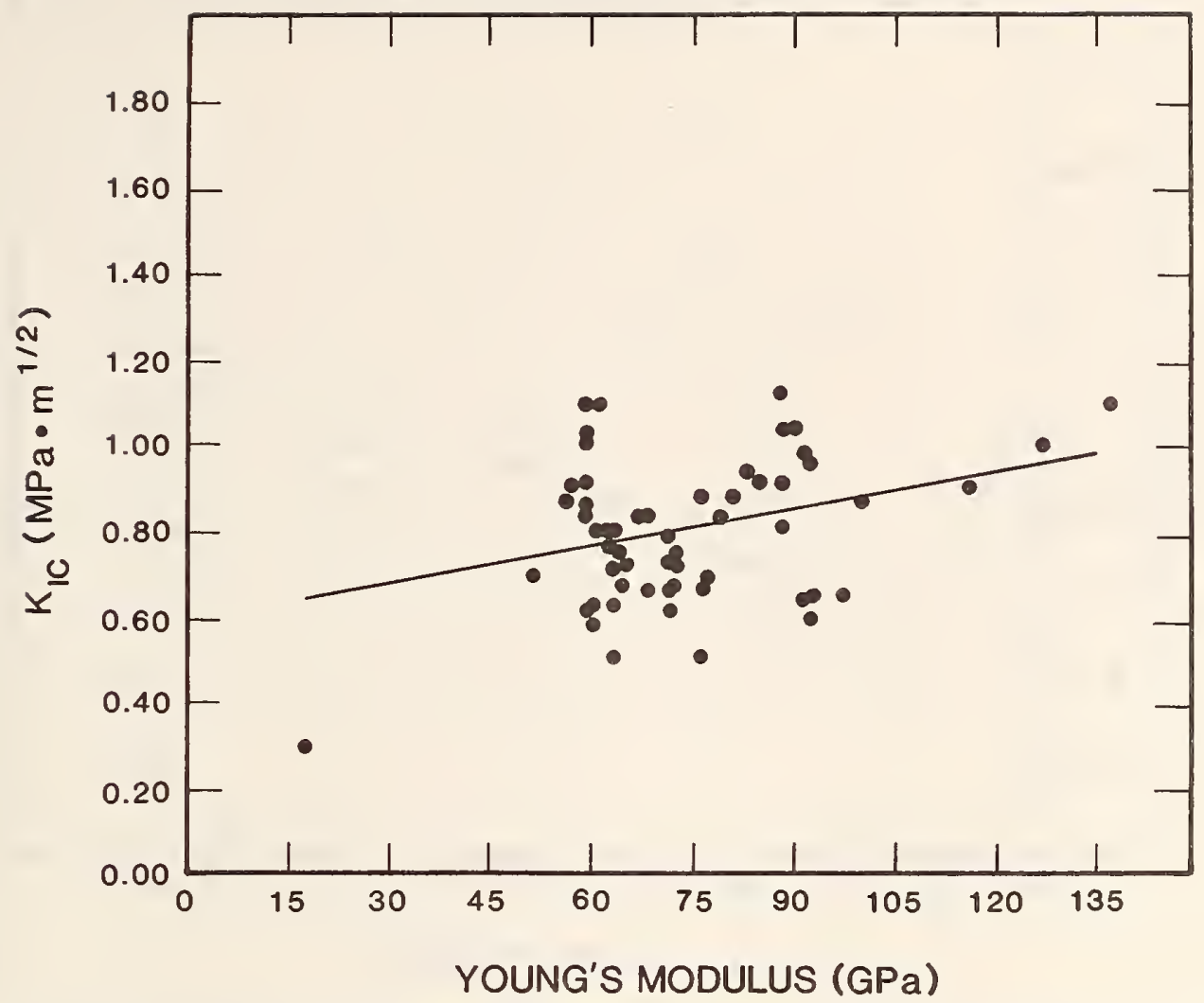

Figure 3. The same data as in figures 1 and 2, plotted as $\mathrm{K}_{\mathrm{IC}}$ as function of Young's modulus. The curve is the best fit to a quadratic equation for $\mathrm{K}_{\mathrm{lC}}$ in terms of Young's modulus.

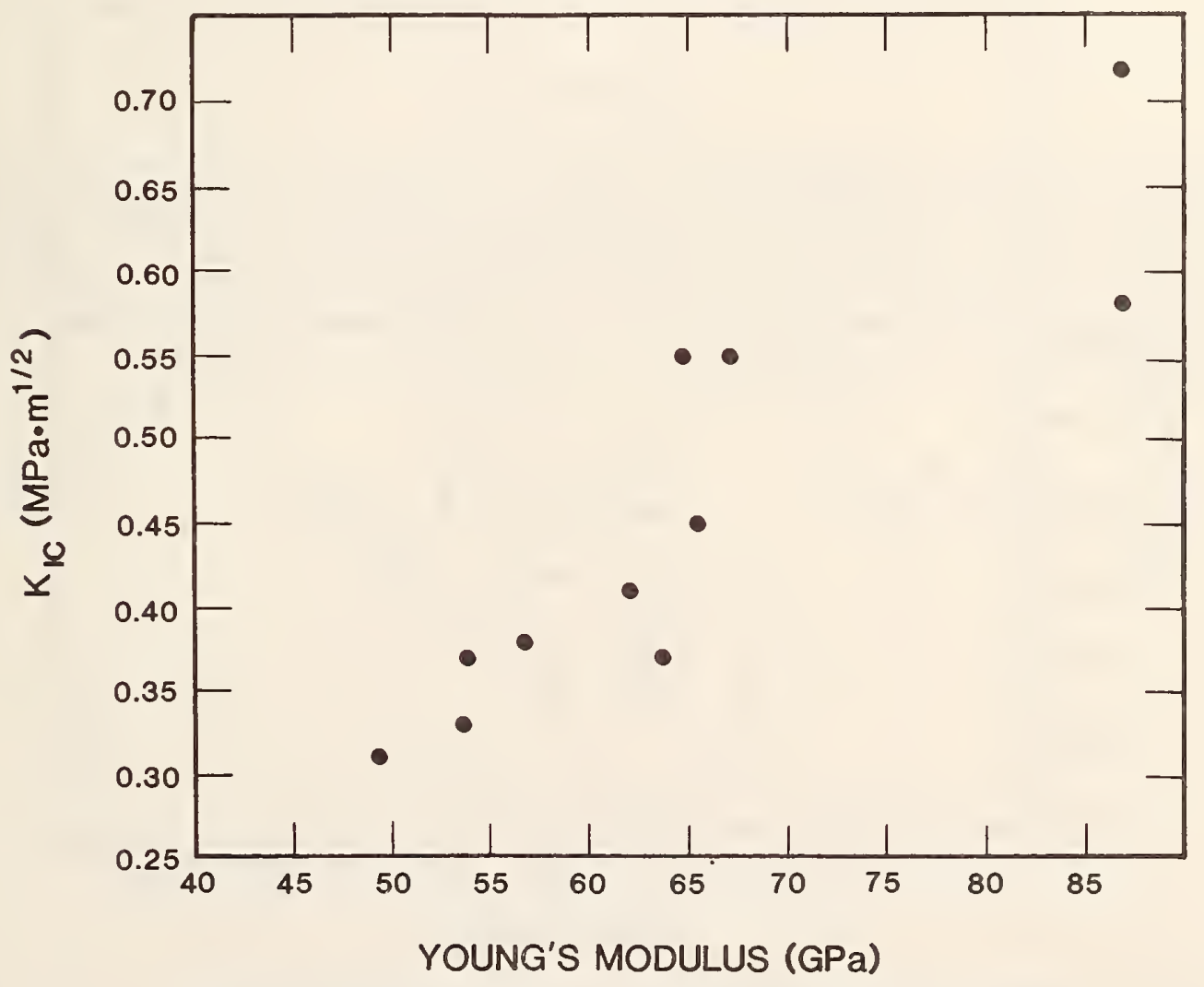

Figure 4. $\mathrm{K}_{\mathrm{IC}}$ plotted versus the Young's modulus of $\mathrm{Al}_{2} \mathrm{O}_{3}-\mathrm{P}_{2} \mathrm{O}_{5}$ glasses. 


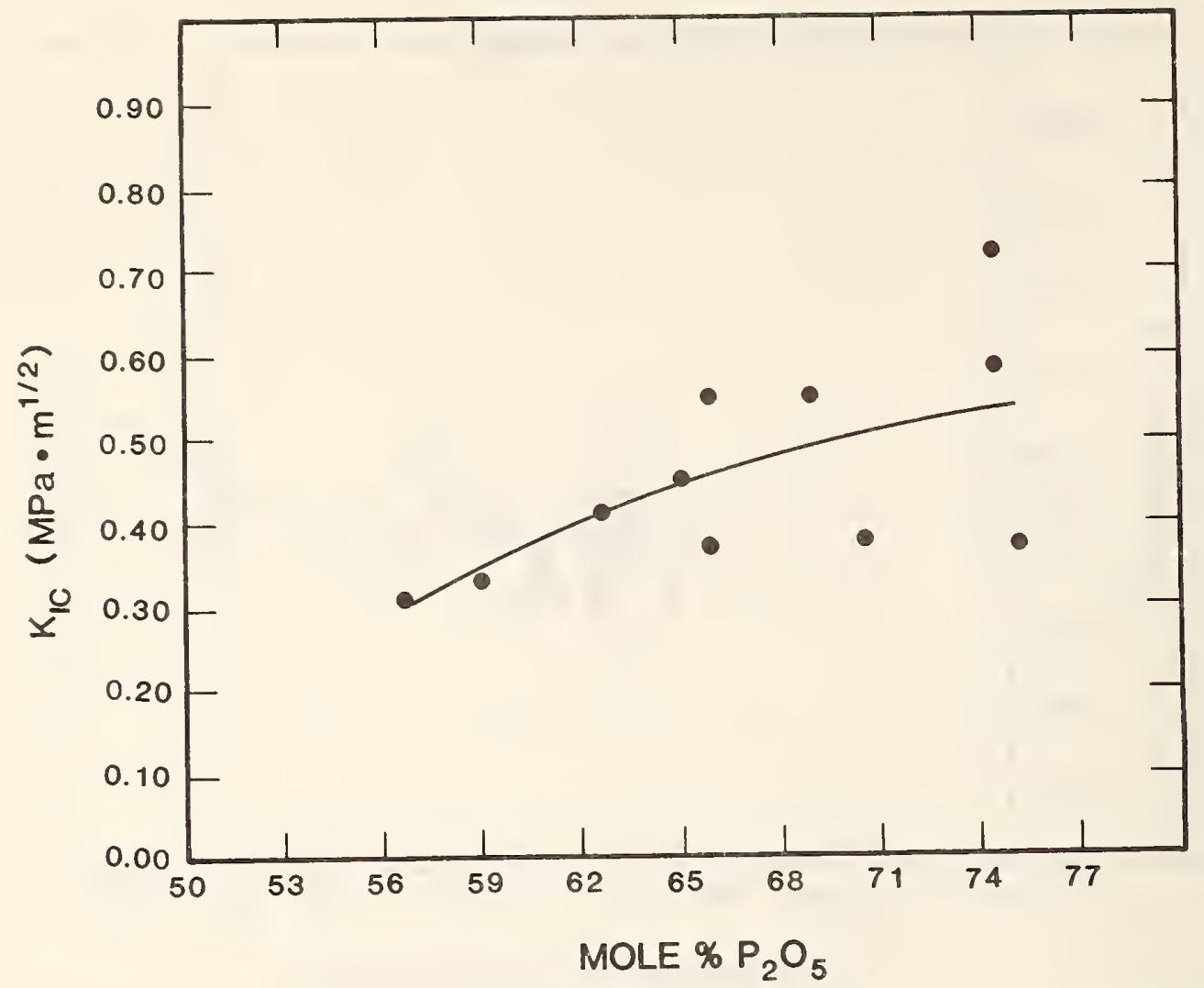

Figure 5. The same data shown in figure 4 plotted as $\mathrm{K}_{\mathrm{IC}}$ versus the mole $\% P_{2} \mathrm{O}_{5}$.

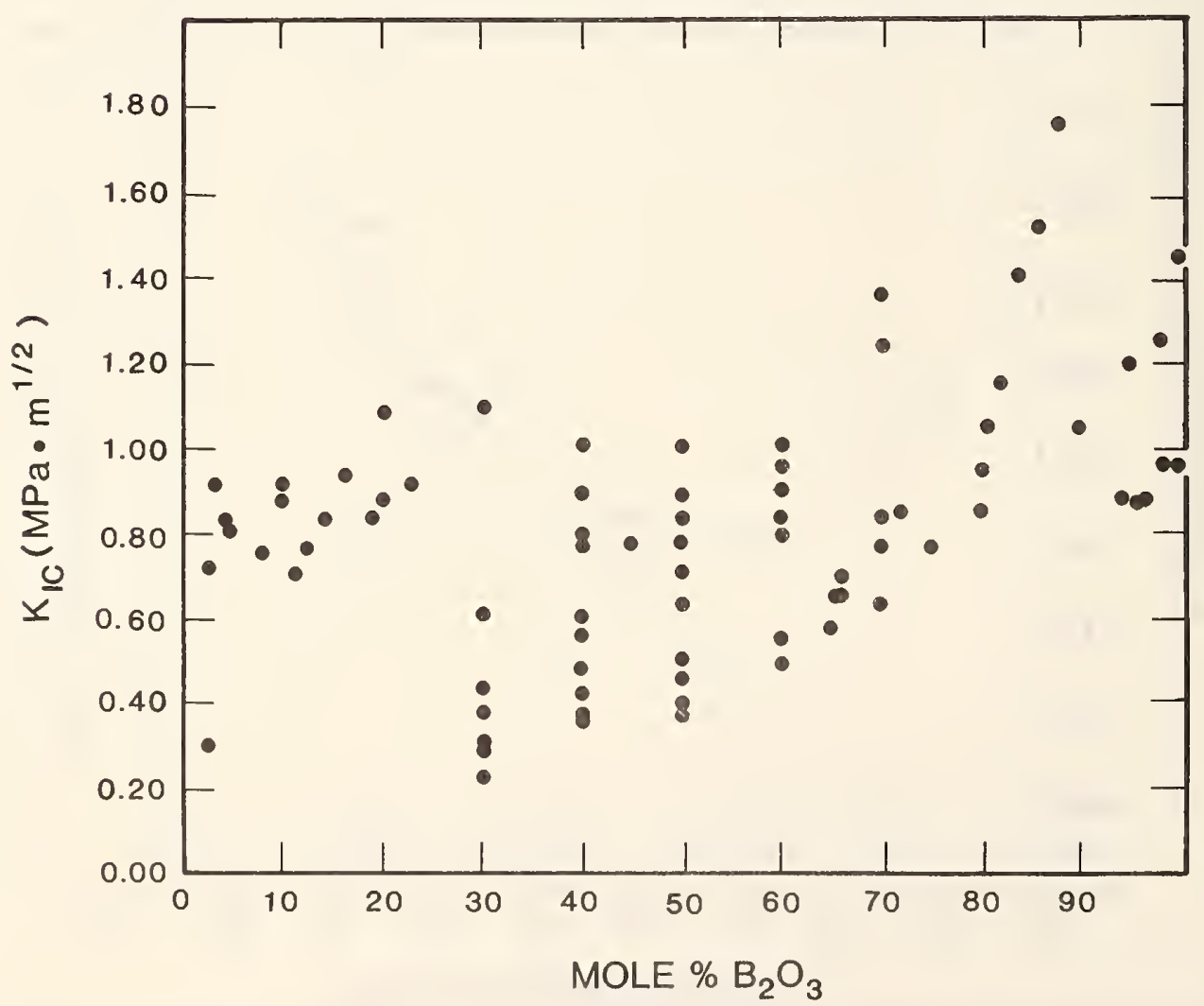

Figure 6. $\mathrm{K}_{\mathrm{IC}}$ plotted against the mole $\% \mathrm{~B}_{2} \mathrm{O}_{3}$ for all $\mathrm{B}_{2} \mathrm{O}_{3}$ containing glasses. 


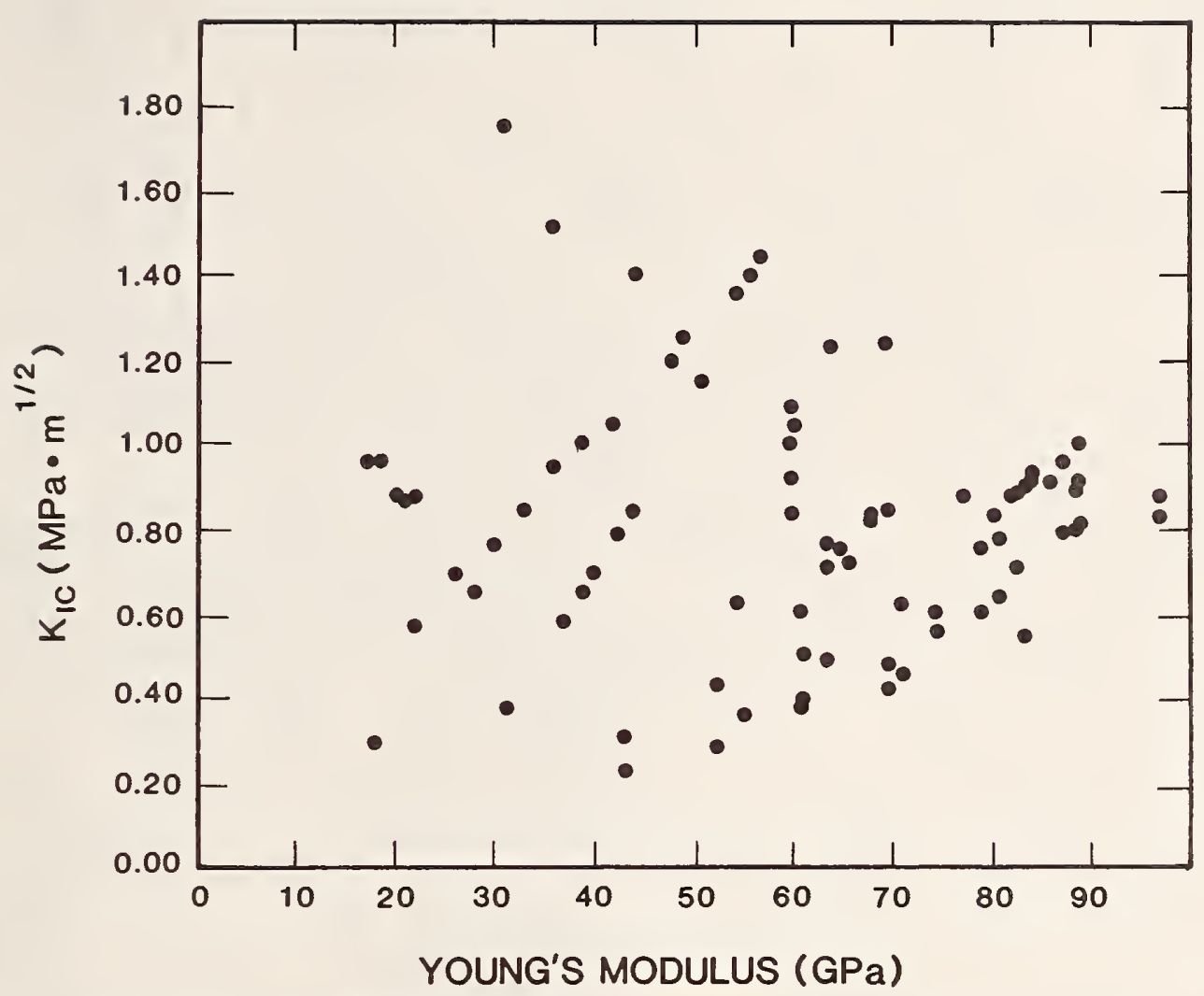

Figure 7. The same data as in figure 6 plotted as $\mathrm{K}_{\mathrm{IC}}$ versus the Young's modulus of the glasses.

\section{Summary}

This paper presents details of a computerized data base of experimentally determined fracture mechanics parameters for oxide glasses. The philosophy behind the format of the system is described. The utility of this system in enabling a designer to choose a glass composition or for understanding fracture behavior is demonstrated through the presentation of plots of $K_{\mathrm{IC}}$ or $\gamma$ as a function of glass composition or elastic modulus.

Finally, the system is capable of providing plots of $n$ versus composition or Young's modulus. However, the values of $n$ are quite sensitive to test environment as well, so this variable must be accounted for in any analysis. 


\begin{tabular}{|c|c|c|c|}
\hline $\begin{array}{l}\text { U.S. DEPT. OF COMM. } \\
\text { BIBLIOGRAPHIC DATA } \\
\text { SHEET (See instructions) }\end{array}$ & $\begin{array}{l}\text { 1. PUBLICATION OR } \\
\text { REPORT NO. } \\
\text { NBS/TN- } 1212\end{array}$ & 2. Performing Organ. Report No. & $\begin{array}{l}\text { 3. Publication Date } \\
\text { June } 1985\end{array}$ \\
\hline \multicolumn{4}{|c|}{ 4. TITLE AND SUBTITLE } \\
\hline \multicolumn{4}{|c|}{$\begin{array}{l}\text { 5. AUTHOR(S) } \\
\text { S. W. Freiman, T. L. Baker, and J. B. Wachtman, Jr. }\end{array}$} \\
\hline \multicolumn{3}{|c|}{ 6. PERFORMING ORGANIZATION (If joint or other than NBS, see instructions) } & $\begin{array}{l}\text { 7. Contract/Grant No. } \\
\text { 8. Type of Report \& Period Covered } \\
\text { Final }\end{array}$ \\
\hline
\end{tabular}

9. SPOTSORHG ORGANIZATION IHAEE ANC COMPLETE ADORESS (Street, City. StOte, ZHP)

Same as 6 .

10. SUPPLEMENTARY NOTES

Document describes a computer program; SF-185, FIPS Software Summary, is attached.

11. ABSTRACT (A 200-word or less factual summary of most significant information. If document includes a significant bibliography or literature survey. mention it here)

Values of critical fracture toughness $\left(\mathrm{K}_{\mathrm{IC}}\right)$, fracture energy ( $\left.\boldsymbol{\gamma}\right)$, subcritical crack growth exponents ( $n$ ) and Young's modulus (E), are compiled and tabulated for a wide variety of oxide glasses. A computerized data retrieval system has been formulated to allow for selection of data by either glass composition, investigator, or experimental technique, and year. Plotting routines allow $\mathrm{K}_{\mathrm{IC}}$ or $\boldsymbol{\gamma}$ to be plotted versus the mole \% of a particular component or the Young's modulus of the glass. A few illustrations are given to demonstrate trends in $\mathrm{K}_{\mathrm{IC}}$ and $\gamma$ as a function of composition and elastic modulus.

12. KEY WORDS (Six to twelve entries; alphabetical order: capitalize only proper names; and separate key words by semicolons) crack growth exponents; elastic modulus; fracture database; fracture mechanics; fracture toughness; oxide glasses

13. AVAILABILITY

\section{Unlimited}

For Official Distribution. Do Not Release to NTIS

X Order From Superintendent of Documents, U.S. Government Printing Office, Washington, DC 20402.

Order From National Technical Information Service (NTIS), Springfield, VA 22161
14. NO. OF PRINTED PAGES

\section{1}

15. Price 


\section{Periodical}

Journal of Research-The Journal of Research of the National Bureau of Standards reports NBS research ana development in those disciplines of the physical and engineering sciences in which the Bureau is active. These include physics, chemistry, engineering, mathematics, and computer sciences. Papers cover a broad range of subjects, with major emphasis on measurement methodology and the basic technology underlying standardization. Also included from time to time are survey articles on topics closely related to the Bureau's technical and scientific programs. Issued six times a year.

\section{Nonperiodicals}

Monographs-Major contributions to the technical literature on various subjects related to the Bureau's scientific and technical activities.

Handbooks-Recommended codes of engineering and industrial practice (including safety codes) developed in cooperation with interested industries, professional organizations, and regulatory bodies.

Special Publications - Include proceedings of conferences sponsored by NBS, NBS annual reports, and other special publications appropriate to this grouping such as wall charts, pocket cards, and bibliographies.

Applied Mathematics Series-Mathematical tables, manuals, and studies of special interest to physicists, engineers, chemists, biologists, mathematicians, computer programmers, and others engaged in scientific and technical work.

National Standard Reference Data Series-Provides quantitative data on the physical and chemical properties of materials, compiled from the world's literature and critically evaluated. Developed under a worldwide program coordinated by NBS under the authority of the National Standard Data Act (Public Law 90-396).

NOTE: The Journal of Physical and Chemical Reference Data (JPCRD) is published quarterly for NBS by the American Chemical Society (ACS) and the American Institute of Physics (AIP). Subscriptions, reprints, and supplements are available from ACS, 1155 Sixteenth St., NW, Washington, DC 20056.

Building Science Series-Disseminates technical information developed at the Bureau on building materials, components, systems, and whole structures. The series presents research results, test methods, and performance criteria related to the structural and environmental functions and the durability and safety characteristics of building elements and systems.

Technical Notes-Studies or reports which are complete in themselves but restrictive in their treatment of a subject. Analogous to monographs but not so comprehensive in scope or definitive in treatment of the subject area. Often serve as a vehicle for final reports of work performed at NBS under the sponsorship of other government agencies.

Voluntary Product Standards-Developed under procedures published by the Department of Commerce in Part 10, Title 15, of the Code of Federal Regulations. The standards establish nationally recognized requirements for products, and provide all concerned interests with a basis for common understanding of the characteristics of the products. NBS administers this program as a supplement to the activities of the private sector standardizing organizations.

Consumer Information Series-Practical information, based on NBS research and experience, covering areas of interest to the consumer. Easily understandable language and illustrations provide useful background knowledge for shopping in today's technological marketplace.

Order the above NBS publications from: Superintendent of Documents, Government Printing Office, Washington, DC 20402.

Order the following NBS publications-FIPS and NBSIR's-from the National Technical Information Service, Springfield, VA 22161.

Federal Information Processing Standards Publications (FIPS PUB)-Publications in this series collectively constitute the Federal Information Processing Standards Register. The Register serves as the of ficial source of information in the Federal Government regarding standards issued by NBS pursuant to the Federal Property and Administrative Services Act of 1949 as amended, Public Law 89-306 (79 Stat. 1127), and as implemented by Executive Order 11717 (38 FR 12315, dated May 11, 1973) and Part 6 of Title 15 CFR (Code of Federal Regulations).

NBS Interagency Reports (NBSIR)-A special series of interim or final reports on work performed by NBS for outside sponsors (both government and non-government). In general, initial distribution is handled by the sponsor; public distribution is by the National Technical Information Service, Springfield, VA 22161, in paper copy or microfiche form. 
U.S. Department of Commerce

National Bureau of Standards

Gaithersburg, MD 20899

Official Business

Penalty for Private Use $\$ 300$ 